\title{
Attosecond physics at the nanoscale
}

This content has been downloaded from IOPscience. Please scroll down to see the full text.

2017 Rep. Prog. Phys. 80054401

(http://iopscience.iop.org/0034-4885/80/5/054401)

View the table of contents for this issue, or go to the journal homepage for more

Download details:

IP Address: 130.183.90.175

This content was downloaded on 09/05/2017 at 14:52

Please note that terms and conditions apply.

You may also be interested in:

High energy photoelectron emission from gases using plasmonic enhanced near-fields M F Ciappina, T Shaaran, R Guichard et al.

Advances in attosecond science

Francesca Calegari, Giuseppe Sansone, Salvatore Stagira et al.

Numerical simulation of attosecond nanoplasmonic streaking

E Skopalová, D Y Lei, T Witting et al.

The birth of attosecond physics and its coming of age

Ferenc Krausz

Isolated few-attosecond emission in a multi-cycle asymmetrically nonhomogeneous two-color laser field

Chao Yu, Yunhui Wang, Xu Cao et al.

Spatial shaping of intense femtosecond beams for the generation of high-energy attosecond pulses E Constant, A Dubrouil, O Hort et al.

Attosecond imaging of XUV-induced atomic photoemission and Auger decay in strong laser fields S Zherebtsov, A Wirth, T Uphues et al. 


\title{
Report on Progress
}

\section{Attosecond physics at the nanoscale}

\author{
M F Ciappina ${ }^{1,2}$, J A Pérez-Hernández ${ }^{3}$, A S Landsman ${ }^{4}$, W A Okell ${ }^{1}$, \\ S Zherebtsov ${ }^{1}$, B Förg ${ }^{1}$, J Schötz ${ }^{1}$, L Seiffert ${ }^{5}$, T Fennel ${ }^{5}$, T Shaaran ${ }^{6}$, \\ T Zimmermann ${ }^{7,8}$, A Chacón ${ }^{9}$, R Guichard ${ }^{10}$, A Zaïr ${ }^{11}$, J W G Tisch ${ }^{11}$, \\ J P Marangos ${ }^{11}$, T Witting ${ }^{11}$, A Braun ${ }^{11}$, S A Maier ${ }^{11}$, L Roso ${ }^{3}$, \\ M Krüger ${ }^{1,12,13}$, P Hommelhoff ${ }^{1,12,14}$, M F Kling ${ }^{1,15}$, F Krausz ${ }^{1,15}$ \\ and $M$ Lewenstein ${ }^{9,16}$
}

${ }^{1}$ Max-Planck-Institut für Quantenoptik, Hans-Kopfermann-Str. 1, D-85748 Garching, Germany

2 Institute of Physics of the ASCR, ELI-Beamlines project, Na Slovance 2, 18221 Prague, Czech Republic

3 Centro de Láseres Pulsados (CLPU), Parque Científico, E-37185 Villamayor, Salamanca, Spain

${ }^{4}$ Max-Planck-Institut für Physik komplexer Systeme, Nöthnitzer Str. 38, D-01187 Dresden, Germany

5 Physics Department, University of Rostock, D-18051 Rostock, Germany

${ }^{6}$ Max-Planck-Institut für Kernphysik, Saupfercheckweg 1, D-69117 Heidelberg, Germany

7 Seminar for Applied Mathematics, ETH Zurich, CH-8093 Zurich, Switzerland

${ }^{8}$ Max Planck-POSTECH Center for Attosecond Science, Pohang, Gyeongbuk 37637, Korea

${ }^{9}$ ICFO_Institut de Ciències Fotòniques, The Barcelona Institute of Science and Technology, 08860

Castelldefels (Barcelona), Spain

${ }^{10}$ Department of Physics and Astronomy, University College London, Gower Street, London WC1E 6BT, United Kingdom

11 Blackett Laboratory, Imperial College, London SW7 2AZ, United Kingdom

12 Department für Physik, Friedrich-Alexander-Universität Erlangen-Nürnberg, Staudtstrasse 1, D-91058 Erlangen, Germany

13 Department of Physics of Complex Systems, Weizmann Institute of Science, 76100 Rehovot, Israel

14 Max-Planck-Insitut für die Physik des Lichts, Staudtstrasse 2, D-91058 Erlangen, Germany

15 Fakultät für Physik, Ludwig-Maximilians-Universität München, Am Coulombwall 1, D-85748

Garching, Germany

16 ICREA, Pg. Lluís Companys 23, 08010 Barcelona, Spain

E-mail: marcelo.ciappina@eli-beams.eu

Received 5 August 2014, revised 21 November 2016

Accepted for publication 6 January 2017

Published 20 March 2017

Corresponding Editor Professor Masud Mansuripur

\begin{abstract}
Recently two emerging areas of research, attosecond and nanoscale physics, have started to come together. Attosecond physics deals with phenomena occurring when ultrashort laser pulses, with duration on the femto- and sub-femtosecond time scales, interact with atoms, molecules or solids. The laser-induced electron dynamics occurs natively on a timescale down to a few hundred or even tens of attoseconds ( 1 attosecond $=1$ as $=10^{-18} \mathrm{~s}$ ), which is comparable with the optical field. For comparison, the revolution of an electron on a 1s orbital of a hydrogen atom is $\sim 152$ as. On the other hand, the second branch involves the manipulation and engineering of mesoscopic systems, such as solids, metals and dielectrics, with nanometric precision. Although nano-engineering is a vast and well-established research field on its own, the merger with intense laser physics is relatively recent.
\end{abstract}


In this report on progress we present a comprehensive experimental and theoretical overview of physics that takes place when short and intense laser pulses interact with nanosystems, such as metallic and dielectric nanostructures. In particular we elucidate how the spatially inhomogeneous laser induced fields at a nanometer scale modify the laser-driven electron dynamics. Consequently, this has important impact on pivotal processes such as above-threshold ionization and high-order harmonic generation. The deep understanding of the coupled dynamics between these spatially inhomogeneous fields and matter configures a promising way to new avenues of research and applications. Thanks to the maturity that attosecond physics has reached, together with the tremendous advance in material engineering and manipulation techniques, the age of atto-nanophysics has begun, but it is in the initial stage. We present thus some of the open questions, challenges and prospects for experimental confirmation of theoretical predictions, as well as experiments aimed at characterizing the induced fields and the unique electron dynamics initiated by them with high temporal and spatial resolution.

Keywords: attosecond physics, plasmonic fields, strong field physics

(Some figures may appear in colour only in the online journal)

\section{Introduction}

This report on progress presents a new emerging field of atomic, molecular, and optical physics: atto-nanophysics. It is an area that combines the traditional and already very mature attosecond physics with the equally well developed nanophysics. In the introduction we give just general motivations and description of this new area, restricting ourselves to vary basic (mostly review style) references. An extensive set of references concerning the new area is included in the bulk of the report.

Attosecond physics has traditionally focused on atomic and small molecular targets (Scrinzi et al 2006, Krausz and Ivanov 2009). For such targets the electron excursion amplitude induced by the ultrafast laser pulse is small compared to the wavelength of the driving laser. Hence, the spatial dependence of the laser field can be safely neglected. In the presence of such spatially homogeneous laser fields the time-dependent processes occurring on the attosecond time scale have been extensively investigated (Hentschel et al 2001, Baltuska et al 2003a). This subject has now reached maturity based upon well-established theoretical developments and the understanding of various nonlinear phenomena (see Batani et al (2001), Lewenstein and L'Huillier (2009), Salières et al (1999)), as well as the formidable advances in experimental laser techniques. Nowadays, measurements with attosecond precision are routinely performed in several facilities around the world (for a recent review and perspectives see e.g. Calegari et al (2016a), Krausz (2016)).

At the same time, bulk matter samples have been scaled in size to nanometer dimensions, paving the way to study light-matter interaction in a completely new regime. When a strong laser interacts, for instance, with a metallic structure, it can couple with the plasmon modes inducing the ones corresponding to collective oscillations of free charges. These free charges, driven by the field, generate spots of few nanometers size of highly enhanced near-fields, which exhibit unique temporal and spatial characteristics. The near-fields in turn induce appreciable changes in the local field strength at a scale of the order of tenths of nanometers, and in this way modify the field-induced electron dynamics. In other words, in this regime, the spatial scale on which the electron dynamics takes place is of the same order as the field variations. Moreover, the near-fields change on a sub-cycle timescale as the free charges respond almost instantaneously to the driving laser. As a consequence, we face an unprecedented scenario: the possibility to study and manipulate strong field induced phenomena by rapidly changing fields, which are not spatially homogeneous.

This report on progress is devoted and focused on the experimental and theoretical consequences of spatially inhomogeneous laser driven strong fields in atoms, molecules and nano-structures. We begin with a brief section about attosecond physics. The purpose here is not to describe the subject in detail (for recent review articles on this topic we refer the reader to, e.g. Krausz and Ivanov (2009), Scrinzi et al (2006)), but rather to give a general overview of the strong field processes driven by intense ultrashort laser pulses in optical to mid-IR frequencies. Such pulses are instrumental to all phenomena described here, including high-order harmonic generation (HHG), above-threshold ionization (ATI) and nonsequential double ionization (NSDI).

The following section indicates how our understanding of these strong field processes, relatively well known and studied for atomic gas targets, is affected in the presence of nanoscale condensed matter targets. The emergent field of attosecond physics at the nanoscale marries very fast attosecond processes $\left(1\right.$ as $\left.=10^{-18} \mathrm{~s}\right)$, with very short nanometric spatial scales $\left(1 \mathrm{~nm}=10^{-9} \mathrm{~m}\right)$, bringing a unique and sometimes unexpected perspective on important underlying strong field phenomena.

Section 2 is quite extended and includes a short description of various experimental techniques and methods used in atto-nanophysics, from generation of nano-plasmonic fields, 
design of nano-structures, to more general techniques of super intense laser physics: generation of few-cycle phase stabilized laser pulses, generation of attosecond pulses via HHG, and combining both on attosecond streaking. In this section we present as an excellent example a case study of the Imperial College attosecond beamline and its applications of attostreaking at surfaces.

Section 3 is devoted to the discussion of electron emission imaging from isolated nanoparticles, a subject which has grown in importance and maturity in the last 5 years or so. Similarly hot subject: attosecond control of electrons at nanoscale needle tips is the subject of section 4. Section 5 describes specific aspect of attosecond streaking in nanolocalized plasmonic fields, originating both from isolated nanospheres as well as from nanoantennas. In section 6 we turn to the discussion of experiments on extreme XUV generation by atoms in plasmonic nanofields.

Theoretical approaches are summarized in section 7 , while selected theoretical predictions concerning HHG are presented in section 8 . Section 9 is exclusively devoted to theoretical predictions concerning ATI driven by spatially inhomogeneous fields, while in section 10 we briefly mention other processes of interest, such as multielectron effects and multielectron ionization.

We conclude in a short section 11, stressing the explosive character of the recent development of the atto-nanophysics, and quoting examples of very recent breakthrough papers.

\subsection{Strong field phenomena driven by spatially homogene- ous fields}

A common way of initiating electronic dynamics in atoms or molecules is to expose these systems to an intense and coherent electromagnetic radiation. This interplay results in a variety of widely studied and important phenomena, which we simply list and shortly describe in this section. To put the relevant laser parameters into context, it is useful to compare them with an atomic reference. In the present context, laser fields are considered intense when their strength is not much smaller or even comparable to the Coulomb field experienced by an atomic electron. The Coulomb field in an hydrogen atom is approximately $5 \times 10^{9} \mathrm{~V} \mathrm{~cm}^{-1}(\approx 514 \mathrm{~V}$ $\left.\mathrm{nm}^{-1}\right)$, corresponding to an equivalent intensity of $3.51 \times 10^{16}$ $\mathrm{W} \mathrm{cm}^{-2}$ - this last value actually defines the atomic unit of intensity. With regard to time scales, we note that in the Bohr model of hydrogen atom, the electron takes about 150 as to orbit around the proton, defining the characteristic time for electron dynamics inside atoms and molecules (Corkum and Krausz 2007). Finally, the relevant laser sources are typically in the near-IR regime, and hence laser frequencies are much below the ionisation potential. In particular, an $800 \mathrm{~nm}$ source corresponds to a photon energy of $0.057 \mathrm{au}(1.55 \mathrm{eV})$, which is much below the ionisation potential of hydrogen, given by $1 / 2 \mathrm{au}(13.6 \mathrm{eV})$. At the same time, laser intensities are in the $10^{13}-10^{15} \mathrm{~W} \mathrm{~cm}^{-2}$ range: high enough to ionize some fraction of the sample, but low enough to avoid space charge effects.

While the physics of interactions of atoms and molecules with intense laser pulses is quite complex, much can be understood using theoretical tools developed over the past decades, starting with the seminal work by Keldysh in the 1960's (Keldysh 1965, Perelomov et al 1966, Reiss 1980, Ammosov et al 1986, Faisal 1987). According to the Keldysh theory, an electron can be freed from an atomic or molecular core either via tunnel or multiphoton ionization. These two regimes are characterized by the Keldysh parameter:

$$
\gamma=\omega_{0} \frac{\sqrt{2 I_{\mathrm{p}}}}{E_{0}}=\sqrt{\frac{I_{\mathrm{p}}}{2 U_{p}}},
$$

where $I_{\mathrm{p}}$ is the ionization potential, $U_{p}$ is the ponderomotive energy, defined as $U_{p}=E_{0}^{2} / 4 \omega_{0}^{2}$ where $E_{0}$ is the peak laser electric field and $\omega_{0}$ the laser carrier frequency. The adiabatic tunnelling regime is then characterized by $\gamma \ll 1$, whereas the multiphoton ionization regime by $\gamma \gg 1$. In the multiphoton regime ionisation rates scale as laser intensity $I^{N}$, where $N$ is the order of the process, i.e. the number of photon necessary to overpass the ionization potential.

Many experiments take place in an intermediate or crossover region, defined by $\gamma \sim 1$ (Landsman and Keller 2015). Another way to interpret $\gamma$ is to note that $\gamma=\tau_{T} / \tau_{L}$, where $\tau_{T}$ is the Keldysh time (defined as $\tau_{T}=\frac{\sqrt{2 I_{\mathrm{p}}}}{E_{0}}$ ) and $\tau_{L}$ is the laser period. Hence $\gamma$ serves as a measure of non-adiabaticity by comparing the response time of the electron wavefunction to the period of the laser field.

When laser intensities approach $10^{13} \sim 10^{14} \mathrm{~W} \mathrm{~cm}^{-2}$, the usual perturbative scaling observed in the multiphoton regime $(\gamma \gg 1)$ does not hold, and the emission process becomes dominated by tunnelling $(\gamma<1)$. In this regime a strong laser field bends the binding potential of the atom creating a penetrable potential barrier. The ionization process is governed thus by electrons tunnelling through this potential barrier, and subsequently interacting 'classically' with the strong laser field far from the parent ion (Corkum 1993, Schafer et al 1993, Lewenstein et al 1994).

This concept of tunnel ionization underpins many important theoretical advances, which have received spectacular experimental confirmation with the development of intense ultra-short lasers and attosecond sources over the past two decades. On a fundamental level, theoretical and experimental progress opened the door to the study of basic atomic and molecular processes on the attosecond time scale. On a practical level, this led to the development of attosecond high frequency extreme ultraviolet and x-ray sources, which promise many important applications, such fine control of atomic and molecular reactions among others. The very fact that we deal here with sources that produce pulses of attosecond duration is remarkable. Attosecond XUV pulses allow in principle to capture all processes underlying structural dynamics and chemical reactions, including electronic motion coupled to nuclear dynamics. They allow also to address basic unresolved and controversial questions in quantum mechanics, such as for instance the duration of the strong field ionization process or the tunnelling time (Landsman and Keller 2015, Pazourek et al 2015). 
As was already mentioned, among the variety of phenomena which take place when atomic systems are driven by coherent and intense electromagnetic radiation, the most notable examples are HHG, ATI and NDSI. All these processes present similarities and differences, which we describe briefly below (Batani et al 2001, Lewenstein and L'Huillier 2009, Joachain et al 2012)

HHG takes place whenever an atom or molecule interacts with an intense laser field of frequency $\omega_{0}$, producing radiation of higher multiples of the fundamental frequency $K \omega_{0}$, where in the simplest case of rotationally symmetric targets $K$ is an odd integer. HHG spectra present very distinct characteristics: there is a sharp decline in conversion efficiency followed by a plateau in which the harmonic intensity hardly varies with the harmonic order $K$, and eventually an abrupt cutoff. For an inversion symmetric medium (such as all atoms and some molecules), only odd harmonics of the driving field have been observed because of dipole selection rules and the central symmetric character of the potential formed by the laser pulse and the atomic field. The discovery of this plateau region in HHG has made generation of coherent XUV radiation using table-top lasers possible. The above mentioned features characterize a highly nonlinear process (L'Huiller et al 1993). Furthermore, HHG spectroscopy (i.e. the measurement and interpretation of the HHG emission from a sample) has been widely applied to studying the ultrafast dynamics of molecules interacting with strong laser fields (see, e.g. Marangos (2016)).

Conceptually, HHG is easily understood using the threestep model (Kuchiev 1987, van Linden van den Heuvell and Muller 1988, Corkum 1993, Kulander et al 1993, Lewenstein et al 1994): (i) tunnel ionization due to the intense and low frequency laser field; (ii) acceleration of the free electron by the laser electric field, and (iii) re-collision with the parent ion. The kinetic energy gained by the electron in its journey, under the presence of the laser oscillatory electric field, is converted into a high energy photon and can be easily calculated starting from semiclassical assumptions.

HHG has received special attention because it underpins the creation of attosecond pulses and, simultaneously, it exemplifies a special challenge from a theoretical point of view due to the complex intertwining between the Coulomb and external laser fields. Additionally, HHG is a promising way to provide coherent table-top sized short wavelength light sources in the extreme-ultraviolet (XUV) and soft x-ray regions of the spectrum. Nonlinear atom-electron dynamics triggered by focusing intense laser pulses onto noble gases generates broadband high photons whose energy reaches the soft $\mathrm{x}$-ray region. This nonlinear phenomenon requires laser intensities in the range of $10^{14} \mathrm{~W} \mathrm{~cm}^{-2}$, routinely available from Ti:sapphire femtosecond laser amplifiers (Brabec and Krausz 2000).

Another widely studied phenomenon is the above-threshold ionization (ATI). In fact, and from an historical viewpoint, it was the first one to be considered as a strong nonperturbative laser-matter interaction process (Agostini et al 1979, Muller et al 1986). Conceptually, ATI is similar to HHG, except the electron does not recombine with the parent atom in the step (iii), but rather is accelerated away by the laser field, eventually registered at the detector. Hence, ATI is a much more likely process than $\mathrm{HHG}$, although the latter has opened a venue for a larger set of applications and technological developments. Nevertheless, ATI is an essential tool for laser pulse characterization, in particular in a few-cycle pulses regime. Unlike in HHG, where macroscopic effects, such as phase matching, often have to be incorporated to reliably reproduce the experiment, single atom simulations are generally enough for ATI modeling.

In an ordinary ATI experiment, the energy and/or angular distribution of photoelectrons is measured. The ATI spectrum in energy presents a series of peaks given by the formula $E_{p}=(m+s) \omega_{0}-I_{\mathrm{p}}$, where $m$ is the minimum number of laser photons needed to exceed the atomic binding energy $I_{\mathrm{p}}$ and $s$ is commonly called the number of 'above-threshold' photons carried by the electron. This picture changes dramatically when few-cycle pulses are used to drive the media and the ATI energy spectra becomes much richer structurally speaking (Milošević et al 2006).

In this case, we can clearly distinguish two different regions, corresponding to direct and rescattered electrons. The low energy region, given by $E_{k} \lesssim 2 U_{p}$, corresponds to direct electrons or electrons which never come back to the vicinity of the parent atom.

On the other hand, the high energy part of the ATI spectrum $2 U_{p} \lesssim E_{k} \lesssim 10 U_{p}$ is dominated by the rescattered electrons, i.e. the electrons that reach the detector after being rescattered by the remaining ion-core (Paulus et al 1994). The latter are strongly influenced by the absolute phase of a few-cycle pulse and as a consequence they are used routinely for laser pulse characterization (Paulus et al 2003). These two energy limits for both the direct and rescattered electrons, i.e. $2 U_{p}$ and $10 U_{p}$ can be easily obtained invoking purely classical arguments (Salières et al 2001, Becker et al 2002, Milošević et al 2006).

Most of the ATI and HHG experiments use as an interacting media multielectronic atoms and molecules, and recently condensed and bulk matter. Nevertheless, one often assumes that only one valence electron is active and hence determines all the significant features of the strong field laser-matter interaction. The first observations of two-electron effects in ionization by strong laser pulses go back to the famous Anne L'Huillier's 'knee' (L'Huiller et al 1983). This paper and later the influential Paul Corkum's work (Corkum 1993) stimulated the discussion about sequential versus non-sequential ionization, and about a specific mechanism of the latter (shake-off, rescattering, etc). In the last twenty years, and more recently as well, there has been a growing interest in electron correlations, both in single- and multi-electron ionization regimes, corresponding to lower and higher intensities, respectively (see Shiner et al (2011), Smirnova et al (2009), Walker et al (1994)).

One notable example where electron correlation plays an instrumental role is the so-called non-sequential double ionization (NSDI) (Walker et al 1994). It stands in contrast to sequential double (or multiple) ionization, i.e. when the process comprises a sequence of single ionization events, with no correlation between them. NSDI has attracted considerable interest, since it gives direct experimental access to 
electron-electron correlation-something that is famously difficult to analyse both analytically and numerically (for recent review see, e.g. Bergues et al (2015)).

\subsection{Introduction to atto-nanophysics}

The interaction of ultra-short strong laser pulses with extended systems has recently received much attention and led to an advance in our understanding of the attosecond to few-femtosecond electronic and nuclear dynamics. For instance, the interaction of clusters with strong ultrafast laser fields has long been known to lead to the formation of nanoplasmas in which there is a high degree of charge localisation and ultrafast dynamics, with the emission of energetic (multiple $\mathrm{keV}$ ) electrons and highly charged — up to $\mathrm{Xe}^{40+}$ —ions with high energy (MeV scale) (Shao et al 1996, Ditmire et al 1997a, $1997 b$, Tisch et al 1997, Smith et al 1998). Most recently use of short pulses ( $\sim 10 \mathrm{fs}$ ) has succeeded in isolating the electron dynamics from the longer timescale ion dynamics (which are essentially frozen) revealing a higher degree of fragmentation anisotropy in both electrons and ions compared to the isotropic distributions found from longer pulses $(\sim 100 \mathrm{fs})$ (Skopalová et al 2010).

Likewise, interactions of intense lasers with nano-particles, such as micron scale liquid droplets, leads to hot plasma formation. An important role is found for enhanced local fields on the surface of these droplets driving this interaction via 'field hot-spots' (Mountford et al 1998, Donnelly et al 2001, Gumbrell et al 2001, Symes et al 2004, Sumeruk et al 2007a, 2007b).

Furthermore, studies of driving bound and free charges in larger molecules, e.g. collective electron dynamics in fullerenes (Li et al 2015), and in graphene-like structures (Yakovlev et al 2015), proton migration in hydrocarbon molecules (Kübel et al 2016), charge migration in proteins and bio-molecules (Belshaw et al 2012, Calegari et al 2014, 2016 b) could be included in this category. In addition, biological applications of atto-nanophysics could be envisaged, e.g. to explain the DNA-protein interactions in solutions of living cells (Altucci et al 2012), study the induced covalent cross-link between aromatic amino acids and peptides (Leo et al 2013) and characterize the protein-protein interactions in living cells (Itri et al 2016). In turn, laser-driven broad-band electron wavepackets have been used for static and dynamic diffraction imaging of molecules (Blaga et al 2012, Xu et al 2014, Pullen et al 2015), obtaining structural information with sub-nanometer resolution.

Tailored ultra-short and intense fields have also been used to drive electron dynamics and electron or photon emission from (nanostructured) solids (for a recent compilation see e.g. Hommelhoff and Kling (2015)). The progress seen in recent years has been largely driven by advances in experimental and engineering techniques (both in laser technology and in nanofabrication). Among the remarkable achievements in just the latest years are the demonstration of driving electron currents and switching the conductivity of dielectrics with ultrashort pulses (Schiffrin et al 2013, Schultze et al 2013), controlling the light-induced electron emission from nanoparticles
(Zherebtsov et al 2011, Süßmann et al 2015) and nanotips (Krüger et al 2011, Herink et al 2012, Piglosiewicz et al 2014), and the sub-cycle driven photon emission from solids (Ghimire et al 2011, Schubert et al 2014, Luu et al 2015, Vampa et al 2015). Furthermore, the intrinsic electron propagation and photoemission processes have been investigated on their natural, attosecond timescales (Cavalieri et al 2007b, Schultze et al 2010, Neppl et al 2012, Locher et al 2015, Okell et al 2015).

A key feature of light-nanostructure interaction is the enhancement of the electric near-field by several orders of magnitude, and its local confinement on a sub-wavelength scale (Stockman 2011). From a theoretical viewpoint, this field localisation presents a unique challenge: we have at our disposal strong fields that change on a comparable spatial scale of the oscillatory electron dynamics that are initiated by those same fields. As will be shown throughout this contribution, this singular property entails profound consequences in the underlying physics of the conventional strong field phenomena. In particular, it violates one of the main assumptions that modelling of strong field interactions is based upon: the spatial homogeneity of laser fields in the volume of the electronic dynamics under scrutiny.

Interestingly, an exponential growing attraction in strong field phenomena induced by plasmonic-enhanced fields was triggered by the controversial work of Kim et al (2008). These authors claimed to observe efficient HHG from bowtie metallic nanostructures. Although the interpretation of the outcomes was incorrect, this paper definitively stimulated a constant interest in the plasmonic-enhanced HHG and ATI (Park et al 2011, 2013, Sivis et al 2012, 2013, Kim et al 2012, Pfullmann et al 2013).

Within the conventional assumption, both the laser electric field, $E(\mathbf{r}, t)$, and the corresponding vector potential, $A(\mathbf{r}, t)$, are spatially homogeneous in the region where the electron moves and only their time dependence is considered, i.e. $E(\mathbf{r}, t)=E(t)$ and $A(\mathbf{r}, t)=A(t)$. This is a valid assumption considering the usual electron excursion (estimated classically using $\alpha=E_{0} / \omega_{0}^{2}$ ) is bounded roughly by a few nanometers in the near-IR, for typical laser intensities, and several tens of nanometers for mid-IR sources (note that $\alpha \propto \lambda_{0}^{2}$, where $\lambda_{0}$ is the wavelength of the driving laser and $E_{0}=\sqrt{I}$, where $I$ is the laser intensity) (Brabec and Krausz 2000). Hence, electron excursion is very small relative to the spatial variation of the field in the absence of local (or nanoplasmonic) field enhancement (see figure 1(a)). On the contrary, the fields generated using surface plasmons are spatially dependent on a nanometric region (see figure 1(b)). As a consequence, all the standard theoretical tools in the strong field ionization toolbox (ranging from purely classical to frequently used semiclassical and complete quantum mechanical descriptions) have to be re-examined. In this review, we will therefore focus on how the most important and basic processes in strong field physics, such as HHG and ATI, are modified in a new setting of strong field ultrafast phenomena on a nano-scale. Note that the strong field phenomena driven by plasmonic fields could be treated theoretically within a particular flavour of a nondipole approximation, but neglecting completely magnetic 
(a)

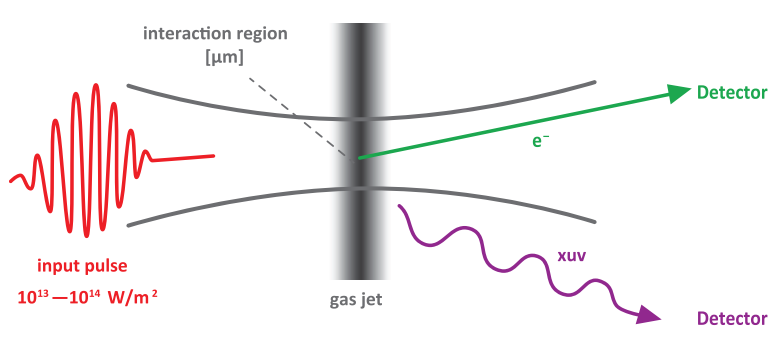

(b)

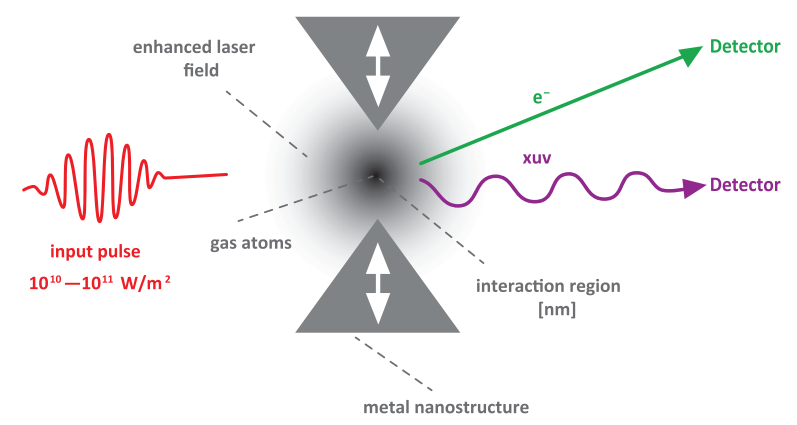

Figure 1. Sketch of conventional (a) and plasmonic-enhanced (b) strong field processes.

effects, by considering we work in a laser intensity range between $10^{13} \sim 10^{15} \mathrm{~W} \mathrm{~cm}^{-2}$ and a driving laser wavelength in the near-infrared (IR) regime $(700 \mathrm{~nm} \sim 1800 \mathrm{~nm})$. For these figures the onset of the magnetic influence starts at $\sim 10^{17}$ $\mathrm{W} \mathrm{cm}{ }^{-2}$ (Joachain et al 2003). We will give more details about this particular point throughout this report.

\section{Experimental tools and techniques}

\subsection{Near-fields and nanoplasmonics}

In this section we will give a brief introduction to nanoplasmonics. Since nanoplasmonics constitutes a vast field of research, we limit our discussion to aspects that are relevant for the attosecond physics discussed in this review.

The interaction of light with matter is naturally confined by the length scales involved - the wavelength of the light and the length associated with the spatial structure of the matter. If the length scale of the structure is much smaller than the wavelength the confinement of the interaction reaches the nano-scale. Electromagnetic near-fields are excited that enable optics below Abbe's diffraction limit. This mechanism opened up the field of nano-optics, also called near-field optics (see, e.g. Maier (2007), Novotny and Hecht (2012), Sarid and Challener (2010) for exhaustive literature on the topic). Nano-optics has found a wide range of applications in microscopy and spectroscopy, among them scanning nearfield microscopy (SNOM) (Wessel 1985, Inouye and Kawata 1994, Hartschuh 2008) and tip-enhanced Raman scattering (TERS) (Wessel 1985, Stöckle Raoul et al 2000).

A prominent and illustrative example of a nano-scale structure used in nano-optics is a nanosphere. In the following we consider the interaction of light of wavelength $\lambda$ with such (a)
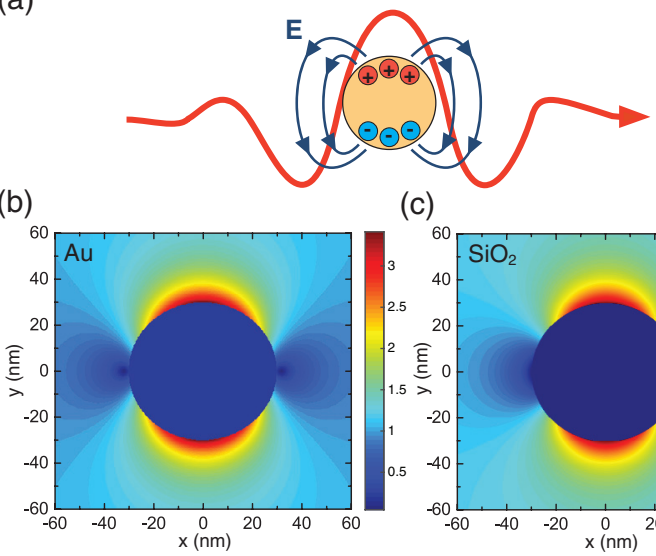

(c)

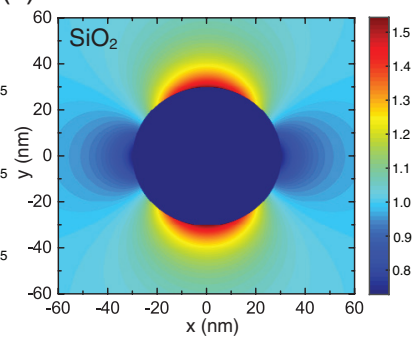

Figure 2. (a) Illustration of an optical near-field (blue) at a nanosphere excited by an external light pulse (red). Adapted from Süßmann et al (2014). (b) Normalized local electric field strength $|\mathbf{E}(\mathbf{r})| /\left|\mathbf{E}_{0}\right|$ at a gold nanosphere with a radius of $30 \mathrm{~nm}$ at an excitation wavelength of $720 \mathrm{~nm}(\epsilon=-16.41+1.38 \mathrm{i})$.

(c) The same for a $\mathrm{SiO}_{2}$ nanosphere $(\epsilon=2.12)$. Figure reused with permission from Süßmann et al (2014). Copyright 2014 by John Wiley \& Sons, Inc.

a sphere made from a linear, local, isotropic material, situated in vacuum. The essential assumption is that the sphere's radius $R$ is much smaller than $\lambda$. In linear optics, described by classical electrodynamics, the properties of the material of the sphere are entirely given by the complex dielectric constant $\epsilon(\lambda)=\epsilon_{\mathrm{r}}(\lambda)+\mathrm{i} \epsilon_{\mathrm{i}}(\lambda)$, which is the square of the material's complex refractive index $n$. The dielectric constant describes the electronic response of the system to external electromagnetic fields. If we now expose the sphere to a homogeneous static electric field, we induce a collective displacement of electric charge along the field direction with respect to the ionic background. When moving from the static field to a linearly polarized light field, this displacement becomes oscillatory, leading to a time-dependent polarization (see figure 2(a)). The sphere now acts as a strongly confined source of light, in other words, as an optical nano-emitter.

For nanospheres with radii $R$ much smaller than the incident wavelength, the quasi-static approximation provides a simple approach to estimate the resulting local electric field. Omitting its time dependence in the quasi-static approximation and neglecting weak magnetic effects, the local field is given by Jackson (1999), Maier (2007)

$$
\mathbf{E}(\mathbf{r})= \begin{cases}\frac{3}{\epsilon+2} \mathbf{E}_{0} & ,|\mathbf{r}|<R, \\ \mathbf{E}_{0}+\frac{3 \hat{\mathbf{r}}(\hat{\mathbf{r}} \cdot \mathbf{p})-\mathbf{p}}{4 \pi \epsilon_{0}|\mathbf{r}|^{3}}, & |\mathbf{r}|>R,\end{cases}
$$

where $\mathbf{r}$ is the spatial vector pointing from the centre of the sphere to the point of interest, $\hat{\mathbf{r}}$ is its unity vector, $\mathbf{E}_{0}$ is the spatially homogeneous incident field and $\epsilon_{0}$ is the vacuum permittivity. The (complex) dipole moment is given by

$$
\mathbf{p}=4 \pi \epsilon_{0} R^{3} \frac{\epsilon-1}{\epsilon+2} \mathbf{E}_{0}
$$


Figures 2(b) and (c) show the normalized local field strength $|\mathbf{E}(\mathbf{r})| /\left|\mathbf{E}_{0}\right|$ for a gold nanosphere and a $\mathrm{SiO}_{2}$ nanosphere, respectively $(R=30 \mathrm{~nm}, \lambda=720 \mathrm{~nm})$. It is evident the optical near-field is spatially inhomogeneous. At the poles the field is strongly enhanced and rapidly decays with increasing distance from the surface, with a 1/e decay constant on the order of the radius $R$ of the sphere. The maximum local field enhancement $\xi=\max |\mathbf{E}(\mathbf{r})| /\left|\mathbf{E}_{0}\right|$, here found at the sphere's poles, is independent of the sphere's radius and is given by

$$
\xi=\left|1+2 \frac{\epsilon-1}{\epsilon+2}\right| .
$$

The enhancement factor for the gold nanosphere at its optical 'hotspots' is $\xi=3.41$. Inside the sphere, the field is uniformly screened and amounts only to a fraction of the strength of the incident field. The example of the nanosphere demonstrates the main characteristics of nano-optics, namely localization, enhancement and screening of electric fields at the nano-scale. In the context of this review, the induced spatial inhomogeneity and the strong enhancement attained at nanostructures is attractive in particular for driving and spatially confining nonlinear processes like low-order harmonic generation (Bouhelier et al 2003, Neacsu et al 2005, Wolf et al 2016) or strong field photoemission (Bormann et al 2010, Schenk et al 2010).

In general, the properties of the excited near-fields critically rely on the polarization of the incident light, the geometry of the nanostructure and on the (wavelength-dependent) dielectric constant of the material $\epsilon(\lambda)$. Depending on these factors, three effects can be distinguished that contribute to near-field excitation and field enhancement (Martin and Girard 1997, Martin et al 2001, Hartschuh 2008). The first effect is geometric in nature and benefits from sharp edges and protrusions of the nanostructure. Under light irradiation, surface charge is accumulated due to the discontinuity of the dielectric constant at the metal-vacuum boundary. This charge in conjunction with the sharp features of the nanostructure leads to strong local electric fields, similar to the electrostatic lightning rod effect. This effect mostly depends on geometry and can be observed for a wide range of materials and wavelength regimes. Prominent examples for nanostructures relying on the geometric effect are nanotips, nanotapers and nanorods. The second effect is observed at nanostructures that are odd multiples of half the wavelength in size. Antenna resonances in the optical domain are excited, leading to strong increase of field enhancement. The third effect is strongly coupled both to material properties and to geometry. Returning to the example of the nanosphere, equation (4) predicts a resonance at $\epsilon_{\mathrm{r}}=-2$. This resonance condition, named Fröhlich condition (Fröhlich and Pelzer 1955), can be fulfilled satisfactorily by plasmonic metals in the visible domain $\left(\epsilon_{\mathrm{r}}<0\right.$ and $\left.0<\epsilon_{\mathrm{i}} \ll\left|\epsilon_{\mathrm{r}}\right|\right)$, such as gold and silver. Such resonances are called localized surface plasmon resonances (LSPRs). Localized surface plasmons are excited that can lead to long-lived charge oscillations and also higher field enhancement than for other materials. Furthermore, propagating plasmon waves can be excited and observed, e.g. at the shank of a nanotip (Berweger et al 2012). This shows that plasmonics can be confined to the nano-scale, enabling nanoplasmonics (see, e.g. Kauranen and Zayats (2012), Sonnefraud et al (2012), Stockman (2011) for review articles on various aspects of nanoplasmonics).

Time-domain effects become very important if the incident light field is pulsed and broadband. Depending on the excitation spectrum in amplitude and phase, on the morphology of the nanostructure and on the wavelength-scaling of the dielectric constant, the induced near-field can be shaped in amplitude and phase and in its spatial behaviour. In particular, plasmonic materials typically feature long-lived plasmon oscillations that persist after the excitation pulse has ended, with lifetimes in the femtosecond domain (see, e.g. Sönnichsen et al (2002)).

Near-field optics is fully described by linear classical electrodynamics as defined by Maxwell equations. Analytical modelling of near-fields with the quasistatic approximation or with Mie theory (Bohren and Huffman 1998), however, is only possible for a few special cases such as nanospheres and nanoellipsoids (Maier 2007), both assumed to be much smaller than the driving wavelength. For larger spheres and ellipsoids, higher-order modes start to contribute to the nearfield and an analytical treatment becomes elusive. Numerical methods need to be applied in order to solve Maxwell equations within the system's defined boundary conditions. Among those methods are the finite elements method (FEM), the finite-difference time-domain (FDTD) approach and the boundary element method (BEM) (Taflove and Hagness 2005). In general, spectroscopic investigations of the optical response of nanostructures agree well with numerical simulations. Nanophotonic devices can be engineered using numerics and tailored to specific needs (see next section). In order to increase field enhancement, oftentimes nanostructure dimers are placed very close to each, for example in a bow-tie configuration (Sivis et al 2013). Due to coupling of the modes of the dimers, the near-field in the gap between the dimers is strongly enhanced compared to that of a single nanostructure. At very small gap sizes, below $1 \mathrm{~nm}$, classical electrodynamics breaks down and quantum effects like electron tunnelling and nonlocal screening set in (Savage et al (2012), Scholl et al (2013)). In such a case, self-consistent theory approaches have to be applied, such as time-dependent density functional theory (Zuloaga et al 2009, Marinica et al 2015), or quantum corrections to classical electrodynamics have to be introduced (see, e.g. Esteban et al (2012)). This limits the achievable field enhancement factor to lower values than the classical prediction, not only in dimer gaps and in other multiparticle systems (Cirac et al 2012), but also very close to the surface of a single nanostructure (Zuloaga et al 2010).

Near-fields are accessible experimentally by various techniques. Nonlinear processes like second-harmonic generation (Neacsu et al 2005) or nonlinear photoemission (Ropers et al 2007b, Thomas et al 2013, Krüger et al 2014) or also attosecond streaking (Süßmann and Kling 2011b) can be employed as means to probe the magnitude of the local field enhancement. The spatial profile of near-fields can be resolved with the help of inelastic electron scattering processes where a tightly focused, high-energy electron beam is passing close to the nanostructure. Possible experimental observables are cathodoluminescence (Vesseur et al 2007, Chaturvedi et al 
2009), incoherent electron energy loss (Nelayah et al 2007, Huth et al 2013, Schröder et al 2015b) or coherent electron energy gain (Barwick et al 2009, Feist et al 2015). All these methods are particularly useful to probe plasmonic resonances and spatial structures like standing waves and optical hotspots.

\subsection{Design and manufacture of nanoscale targets}

2.2.1. Design rules for plasmonic nanostructures. Like many other resonating systems, plasmonic structures are characterized by a strong dispersive response to the probing field, with strong frequency dependence of the scattering and absorption cross-sections and the field enhancement at the vicinity of the nanoantennas (Maier 2007). The peak of the extinction spectrum is known as the localized surface plasmon resonance (LSPR), and its resonance frequency and shape, so as the near-field enhancement and its time-dependent spectral properties are determined by the materials and the geometry (shape and size) of the nanostructures and their surrounding medium (Fernández-García et al 2014, Lorek et al 2015, Choi et al 2016). The LSPR may be affected also by dipolar coupling between adjacent nanostructures and standing waves in periodically assembled structures (Jain et al 2006). The plasma frequency - a material constant depending on its properties (namely: free electron density, electrons effective mass, and the effective electron dumping rate)—sets the upper limit for the frequency at which an LSPR is achievable for a specific material. For applications in the visible and nearinfra red (NIR), a range of suitable plasmonic materials exists (e.g. aluminium, silver, gold, metal-nitrides, semiconductors and transparent conductive oxides (TCO) (Naik et al 2013)), where the choice of the optimized material usually depends on its materials-compatibility with the overall fabrication process, and on its chemical and physical stability of the materials under operating conditions-(e.g. Ag and Al tend to oxidise in free atmosphere; TiN is stable at high temperatures) and to the optical range of interest-(Al can support LSPR at ultraviolet (UV) frequencies; Ag shows stronger resonance than $\mathrm{Au}$ in the visible due to electronic inter-band transitions in the latter (Maier 2007, Fernández-García et al 2014); TCOs have negative real permittivity only for wavelength longer than 1.3-1.5 $\mu \mathrm{m}$ (Naik et al 2013)). The materials surrounding the nanostructures have a strong effect on the frequency of the LSPR which is red shifted with increase of the refractive index (n). Devices that are designed to operate at the visible optical range are usually fabricated on-top of glass-like substrate with $n$ ranging from 1.3 to 1.5 , compare to $n=3.5$ to 4 for typical semiconductors, and use self-assembly monolayer as adhesion promoters rather than chromium of titanium layer (Habteyes et al 2012). Finally, after the selection of materials, the exact shape and dimensions of the nano-structures are designed to tune the plasmonic resonance frequency to the desired spectral range and functionality. The typical dimensions of gold structures designed to operate in the visible to NIR range are at the order of tens to few hundred of nanometers. The last designing step is usually accomplished by means of finite-elements or wave analysis numerical simulations (Veronis and Fan 2007), covering a wide range of design parameters.
2.2.2. Fabrication methods of plasmonic nanostructures arrays. Fabrication methods of metallic nanostructures are differed one from another by the resolution and critical dimension of the written features, accuracy of placement of structures, and speed and costs of the fabrication process. Here we restrict the discussion to methods that allow a precise placement of nanostructures in the substrate. In these techniques (as oppose to colloidal deposition, for example) direct deposition of nanostructured metals on the substrate is hardly feasible, and the patterning is usually done on electron- or photo-resists masks and then transferred into metallic nanostructures, by means of lift-off (where the resists is used a sacrifice layer), or by selective etching of the pre-deposited metallic layer under the patterned resist (which perform as a masking layer). Alternatively, electro-chemical deposition can be used to grow metal on the exposed sections on the resist. The lithography process is illustrated in figure 3(a).

Below we briefly describe four different methods for nanofabrication of plasmonic nanostructures: electron beam lithography (EBL), focused ion-beam (FIB), direct laser writing (DLW) and soft lithography.

In electron beam lithography (EBL), an electron beam emitted from thermionic or field-emission sources, with typical acceleration voltage of $10-100 \mathrm{kV}$, is focused using electromagnetic and electrostatic lenses onto a thin layer of electron-sensitive resist. The primary electrons strike the resist and generate a cascade of secondary electrons with lower energies, which alter the chemical structure of the exposed area of the resist, changing its solubility. The desired patterning is achieved by a selective exposure of the resist using electron deflectors which direct the focused beam to the desired position on the sample. The attainable resolution of EBL patterning is limited to $\sim 10 \mathrm{~nm}$ due to scattering of electrons in the resists leading to unintended exposure (known as proximity effect) of the resist (del Campo and Arzt 2008), and not by the wavelength of the electrons, which is at the order of $1 \AA$ for $10 \mathrm{keV}$ electrons. High resolution EBL resists usually requires relatively high electron exposure dose, which results in a slow patterning time. Nevertheless, due to its high resolution and its practically ultimate flexibility over the design and placement of structures, EBL is currently the workhorse of plasmonic nanostructure nanofabrication.

Focused ion-beam (FIB) is similar to EBL, but with more capabilities: here, gallium ions are emitted from an ion source, and focused and controllably deflected into the sample. Compared to EBL, FIB demonstrates a faster patterning of electron-resists (up to 100 times faster) due to the large number of secondary electrons that are emitted from each collision of the gallium ions in the resists (del Campo and Arzt 2008, Wanzenboeck and Waid 2011). FIB can also be used for direct subtractive patterning (milling), where re-deposition of the milled material is avoided by reaction with reactive gas, and the typically $5 \mathrm{~nm}$ diameter beam can realise structures with feature sizes of $20-30 \mathrm{~nm}$.

Direct laser writing (DLW) (Deubel et al 2004), is a maskless photolithography method that allows a fast writing of flat and 3D structures at a resolution lower than that of electron/ion based techniques. A femtosecond laser is coupled to 


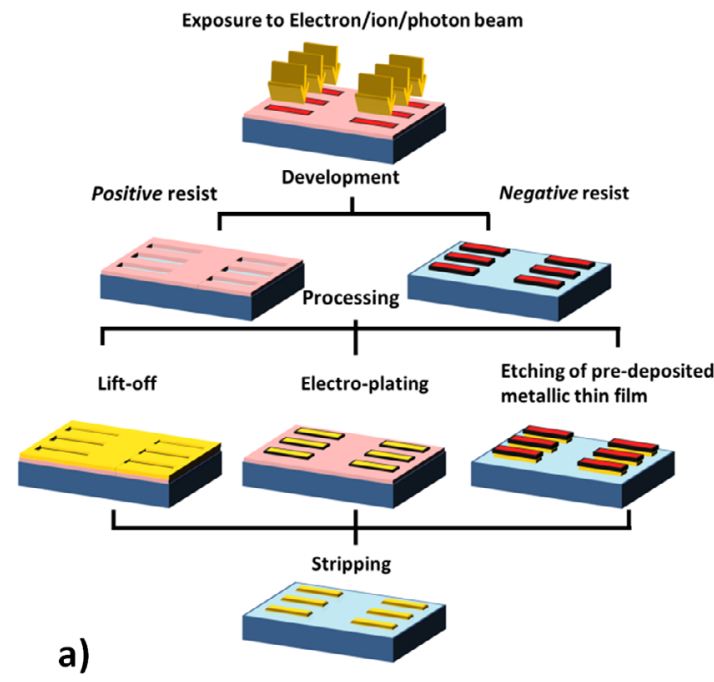

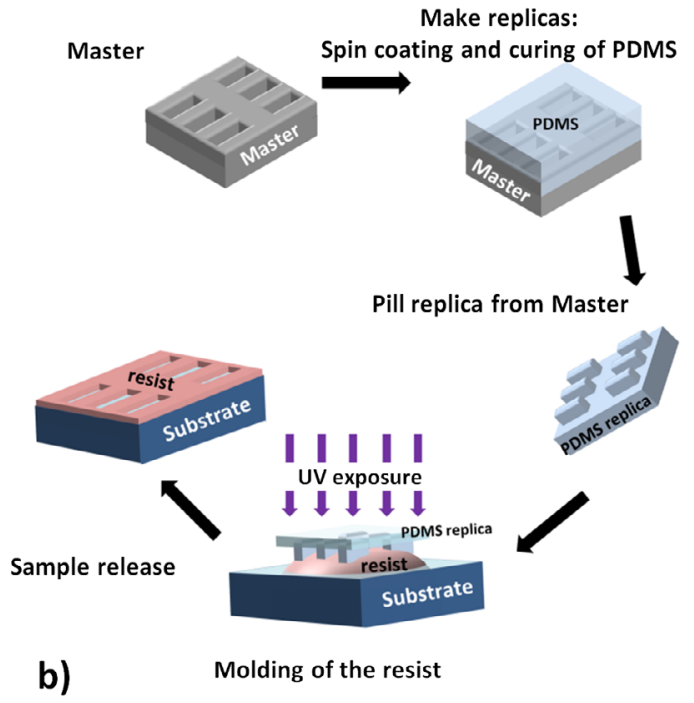

b)

Figure 3. Schematic illustration of the nanofabrication process of plasmonic structures. (a) Complete nanofabrication process: from direct lithography using a beam of photons or charged particles, through chemical development of the resist, post development processing, and stripping of the resists. (b) The moulding lithography process: the hard master is covered with PDMS which is then exposed to UV light, harden, and released from the master. The PDMS replica is pressed into a liquid resist, and the resist is cured by UV light shone through the transparent replica. After stripping, the sample is etched to remove any excess resist. The final outcome is a sample covered with a patterned resist that can be processed is a similar fashion to panel (a).

a microscope and focused via objective with high numerical aperture onto a substrate covered with photosensitive material. High writing speeds of $10-50 \mathrm{~mm} \mathrm{~s}^{-1}$ (Bagheri et al 2015) can be achieved by combining sensitive photo-resists with fast deflection of the light-beam using galvanic mirrors. With these writing speeds, $\mathrm{a} \mathrm{cm}^{2}$ array of nanoantennae can be written by a single source in only few hours. Like many optical lithography systems, the resolution of DLW is diffraction-limited $(\sim 250 \mathrm{~nm}$ for a UV source of $\lambda=405 \mathrm{~nm}$ ). However, a higher resolution of $150 \mathrm{~nm}$ can be achieved when an IR femtosecond laser (usually at $780 \mathrm{~nm}$ ) is used to probe resists which support a nonlinear absorption (namely, two-photon absorption (TPA)).

In contrast to the conventional fabrication methods portrayed above, which are based on exposure of the sample to a beam of photons or charged particles, soft-lithography methods are based on physical contact of the stamp with the substrate (see figure 3(b)). Moulding (embossing) lithography is a form of soft lithography that can be used to print large arrays of plasmonic nanostructures (Qin et al 2010). Nanostructures with feature sizes of $20 \mathrm{~nm}$ and below can be reproducibly fabricated by this technique. Examples of 2D and 3D structures fabricated by the techniques discussed above are presented in figure 4 .

\subsection{Few-cycle carrier envelope phase (CEP) stabilised lasers}

The main enabling technology for strong field and attosecond physics is the ability to generate intense few-cycle laser pulses with stabilized waveforms (Brabec and Krausz 2000). A typical laser system for attosecond science consists of a Ti:sapphire chirped pulse amplification (OPA) system providing $<30 \mathrm{fs}$ pulses at pulse energies around $1 \mathrm{~mJ}$ or above with repetition rates of $1-5 \mathrm{kHz}$. To achieve the few-cycle pulse durations required for attosecond science these pulses are sent through a hollow core capillary (typically $250-400 \mu \mathrm{m}$ inner diameter) for spectral broadening. Mostly an argon or neon gas fill is used. The hollow capillary pulse compression system can be used in static fill mode or in differential pumping/gradient pressure mode, where the gas is supplied at the exit side, whilst the entrance side is kept at vacuum. This improves coupling efficiency and beam quality (Robinson et al 2006). The broadened laser pulses exit the gas filled capillary with a positive chirp. Pulse compression to few-cycle duration is achieved with broadband chirped mirrors combined with thin glass wedges for fine-tuning (see a typical setup in figure 5). The current state of the art of these pulse compression systems are pulses below 4 fs with 0.5-1 mJ level pulse energies. Pulses with 3.8, 3.5, and 4 fs durations and $0.4,0.5$, and $1 \mathrm{~mJ}$ pulse energies at 1 and $4 \mathrm{kHz}$ repetition rate have been produced (Cavalieri et al 2007a, Witting et al 2011, Schweinberger et al 2012, Okell et al 2013).

Tailored electric field waveforms have been produced by a combination of discrete spectral bands derived from a hollow capillary waveguide (Wirth et al 2011). Recently the combination and careful compression led to optical waveforms with attosecond pulse durations (Hassan et al 2016).

Alternative approaches allow the use of non-CEP stabilised laser systems (Schmidt et al 2011). Targeting higher repetition rates is a current area of research. Fibre lasers with postcompression similar to the scheme described above promise to deliver few-cycle laser pulses with repetition rates in the $\mathrm{MHz}$ range (Limpert et al 2011). A viable alternative to achieve amplification of large bandwidths at high repetition rates is the optical parametric chirped pulse amplification (OPCPA) technology. Recently 6 fs pulses at $300 \mathrm{kHz}$ repetition rates have been demonstrated (Prinz et al 2015).

At pulse durations of only a few or even a single optical cycle the phase between the carrier wave and the envelope, the 

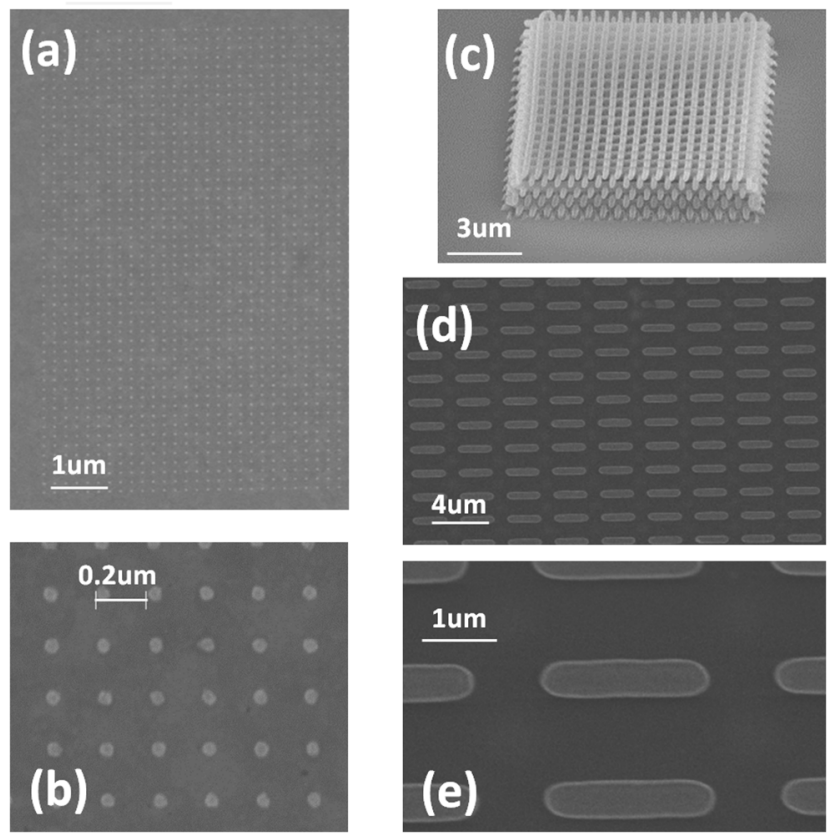

Figure 4. Scanning electron microscopy (SEM) images of nanostructures. ((a)-(b)) Positive EBL writing: $50 \mathrm{~nm}$ thick Au discs with a diameter of $55 \mathrm{~nm}$ made by writing in positive resist (PMMA), followed by thermal evaporation and lift off process. ((c)-(e)) DLW. (c): 3D woodpile structure made of polymeric photoresist coated with $25 \mathrm{~nm}$ of gold. ((d)-(e)): Au antennas with LSPR at the near- and mid-IR regime, fabricated by DLW of positive photo-resist (AZ Mir 701), followed by gold sputtering and lift off process.

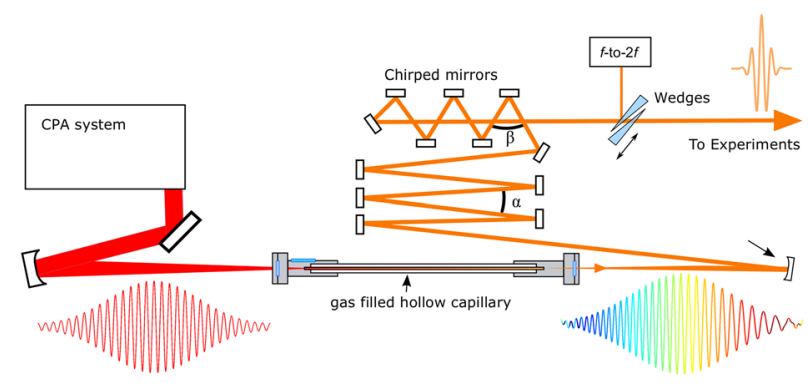

Figure 5. Typical few-cycle laser system with CPA, gas filled hollow capillary for spectral broadening, and compression with chirped mirrors and glass wedges.

so-called carrier envelope phase (CEP), becomes an important parameter in strong field driven interactions and in attosecond pulse generation (Xu et al 1996, Apolonski et al 2000, Dietrich et al 2000, Baltuska et al 2003b, Paulus et al 2003, Luecking et al 2012). The laser electric field thus can be written as:

$$
E(t)=E_{0} f(t) \cos \left[\omega t+\phi_{\mathrm{CEP}}\right],
$$

where $E_{0}$ is the laser electric field peak amplitude, $f(t)$ the pulse envelope and $\phi_{\mathrm{CEP}}$ denotes the CEP (see figure 6).

An important aspect of few-cycle laser systems for attosecond science is the characterization of the generated few-cycle laser pulses. For a long time autocorrelation and especially the inteferometric autocorrelation has been popular. However, despite giving an estimate about the pulse

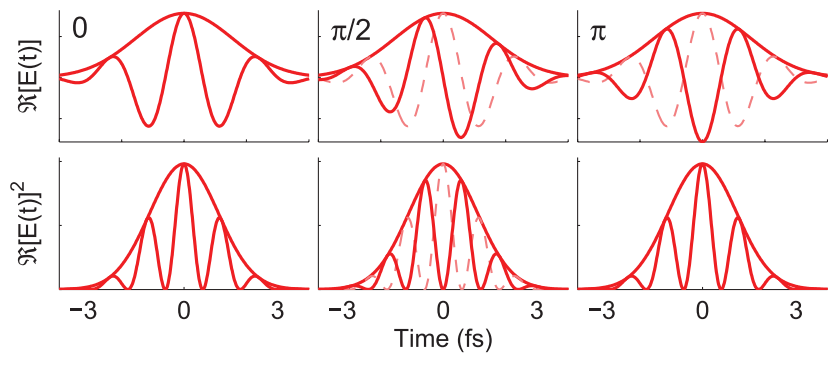

Figure 6. Illustration of the CEP for a few-cycle pulse. Top row: electric field and envelope of three pulses with CEP of $0, \pi / 2$, and $\pi$. Bottom row: field intensity and envelope for the same three pulses. The 'cos' waveforms for CEP 0 and $\pi$ have a single strongest half-cycle, whilst the 'sin' waveform with CEP $\pi / 2$ is characterized by two equally strong neighbouring half-cycles.

duration, autocorrelation cannot recover the full temporal pulse shape (Chung and Weiner 2001). In the last decade a number of advanced metrology methods, able to recover the full complex electric field of ultrashort laser pulses, have been developed. Amongst them are frequency resolved optical gating (FROG) (Kane and Trebino 1993). FROG can be understood as a frequency resolved autocorrelation. The electric field of the unknown laser pulse is recovered with an iterative optimization algorithm. FROG has been employed to measure pulses down to near single cycle pulse durations (Akturk et al 2008). A more recent development is the dispersion scan technique (d-scan). In d-scan an iterative algorithm recovers the electric field of ultrafast laser pulses from a series of second harmonic spectra for a varying amount of dispersion introduced into the unknown pulse (Miranda et al 2012). Another attractive pulse characterization method is spectral phase interferometry for direct electric field reconstruction (SPIDER) (Iaconis and Walmsley 1998). SPIDER relies on self referencing spectral shearing interferometry to characterize the electric field of ultrashort laser pulses. The spectral phase of an unknown laser pulse is recovered from the measured interferogram with a direct algebraic reconstruction algorithm. As a one-dimensional data trace describes a onedimensional laser field $E\left(x_{0}, y_{0}, t\right)$ SPIDER can be extended to multiple spatial dimensions to deliver spatio-temporal information. A variant of SPIDER, that uses spatial encoding of the phase and direct spectral filtering (SEA-F-SPIDER), has been employed to spatio-temporally characterize near-single cycle pulses, as can be seen in figure 7 (Witting et al 2011, Balciunas et al 2015). Recent developments are direct field sampling techniques that employ a strong and short pulse and HHG to sample arbitrary electric field waveforms (Kim et al 2013, Wyatt et al 2016). An excellent review of ultrafast metrology can be found here (Walmsley and Dorrer 2009).

\subsection{Attosecond pulse generation from HHG}

The HHG process, in which high-order harmonics of a strong laser field are generated in its interaction with a gas-phase medium, was introduced in section 1 . The short wavelength emission (VUV-XUV) from HHG can be of attosecond duration and hence HHG lies at the heart of attosecond science. 
The attosecond pulses can be emitted in pulse trains at repetition rates typically in the petahertz range (Antoine et al 1996, Mairesse et al 2003) or as isolated pulses, i.e. one attosecond pulse generated per laser pulse. A key feature of the attosecond pulse emission is its automatic synchronisation with the driving laser pulse. This permits pump-probe experiments using the attosecond pulse and the laser pulse.

Here we concentrate on the generation of isolated attosecond pulses that can be obtained by driving the HHG process with few-cycle CEP stabilised laser pulses, as described in the section 2.3. This method is known as amplitude gating (Hentschel et al 2001, Kienberger et al 2004, Goulielmakis et al 2008, Frank et al 2012), because the highest energy emission from the HHG process is confined to the single, highest amplitude half-cycle of the drive laser pulse. This gating is only possible for a few-cycle pulse for which the field amplitude of neighbouring half-cycles is significantly lower. CEP stabilisation is crucial to ensure the peak of the carrier field coincides with the peak of the envelope (Jones et al 2000, Baltuska et al 2003a). The temporal confinement of the highest energy HHG emission to a single half cycle of the laser field leads to the formation of a spectral continuum at the short wavelength limit (the cutoff) of the HHG spectrum. This continuum region can be spectrally bandpass filtered using submicron thickness foil filters and multi-layer XUV mirrors to produce an isolated attosecond pulse.

Since the first measurement of an isolated sub-femtoscond pulse (650 as at a photon energy of around $90 \mathrm{eV}$ ) produced from HHG in Ne driven by a 7 fs near infra-red (NIR) pulse in 2001 (Hentschel et al 2001), there has been considerable progress in attosecond pulse generation. Using shorter NIR drive pulses, attosecond pulses as short as 80 as have been generated (Goulielmakis et al 2008). A range of techniques have also been developed to allow the generation of isolated attosecond pulses from multi-cycle rather than few-cycle pulses (Altucci et al 2011). Longer drive laser pulses are technically less demanding to produce and can have higher energy than few-cycle pulses, with the potential to generate more intense attosecond pulses. Foremost amongst these techniques is polarisation gating (Corkum et al 1994, Sansone et al 2006, Sola et al 2006). Polarisation gating uses a drive pulse with a time varying ellipticity to confine the HHG emission to a short interval during which the pulse is approximately linearly polarised. Extensions of this technique, known as double optical gating (DOG) (Mashiko et al 2008) and generalised DOG (GDOG) (Feng et al 2009), where polarisation gating is combined with two-colour gating (Mashiko et al 2008), have proven particularly effective for isolated attosecond pulse generation using multi-cycle drive laser pulses. In fact, the current record for the shortest attosecond pulse (67 as) was obtained using DOG (Zhao et al 2012). Another method, known as ionisation gating (Ferrari et al 2010, Pérez-Hernández et al 2011), uses rapid field-ionisation of the generating medium on the rising edge of the laser pulse to confine the HHG emission to a single half cycle.

Isolated attosecond pulses can currently be produced over the spectral range $20-145 \mathrm{eV}$ (the extremes of this range are obtained in Guggenmos et al (2015), Huppert et al (2015)) with durations well below 100 as. Attosecond pulses are emitted with low divergence, spatially-coherent beams that by virtue of their short wavelength and excellent beam quality can be focused to relatively small spots $(<1 \mu \mathrm{m})$. For a linearly polarised drive laser pulse, the attosecond pulse is linearly polarised, but elliptical polarised attosecond pulses have also been predicted using polarisation gating (Henkel et al 2013). Resonant HHG driven by elliptically polarised laser pulses has been shown to deliver quasi-circularly polarised ultrashort pulses in the extreme ultraviolet (Ferré et al 2015), but this has not yet been extended to the attosecond domain.

Attosecond pulse energies are typically in the picojoulenanojoule range and this relatively low photon flux is frequently a limitation in gas-phase experiments, where target densities are typically low and interaction cross sections are often small. For condensed phase targets, including those at the nanoscale, one usually encounters the opposite problem. Due to the high target density, space-charge effects can distort the spectrum and spatial distribution of photoemitted electrons. This typically necessitates a reduction in the ionising photon flux to levels where space-charge effects are negligible. This often leads to low signal count rates that are not so dissimilar to those obtained in gas phase targets. In such circumstances, the use of high repetition rate lasers (hundreds of $\mathrm{kHz}-\mathrm{MHz}$ ) (Limpert et al 2011) is particularly advantageous. With nanoscale targets in particular, care must be taken not to damage the targets through excessive laser fluence. For example, nanoantennae (see section 6) can easily be damaged by melting (Pfullmann et al 2013).

We conclude this section on attosecond sources by noting that free electron lasers (FELs) are soon likely to provide another source of attosecond pulses with a brightness far exceeding current HHG-based system (see, for example, Marangos (2011) and references therein). The unprecedented brightness of femtosecond x-ray pulses from FELS is already being used in coherent diffractive imaging experiments to image the dynamics of individual nanostructures, for example the transient melting of a single gold nanocrystal (Clark et al 2015) and the 3D imaging of lattice dynamics in individual gold nanocrystals (Scholz et al 2013).

\subsection{Attosecond streaking}

Attosecond streaking (Itatani et al 2002, Baltuska et al 2003a, Kienberger et al 2004, Mairesse and Quéré 2005, Goulielmakis et al 2008, Mashiko et al 2008, Witting et al 2012) is one of the most important techniques to measure electron dynamics with attosecond resolution. It features prominently in the nanoscale experiments described in this review. It is important to note that attosecond temporal resolution can also be obtained using HHG spectroscopy, which exploits the sub-cycle dynamics of the HHG process itself to interrogate the generating molecular system (for a comprehensive recent review see Marangos (2016)). The intriguing possibility of applying HHG spectroscopy to molecules chemisorbed at surfaces is being considered by a number of groups as a way of understanding the ultrafast exchange of charge between the molecule and the surface. Such efforts would greatly benefit from localised nanoplasmonic field enhancement. 
In an attosecond streaking experiment, an attosecond pulse (in the VUV-XUV range) generated by HHG and a synchronised laser pulse (typically a few-cycle pulse in the NIR range; usually the drive laser pulse for the HHG) propagating collinearly are focused on a target with a controllable delay between them. The photoelectron wavepacket produced by the attosecond pulse is accelerated in the laser field, which is known as the streaking field. This imprints sub-cycle timing information on the photoelectron spectrum. A streaking trace $S(E, \tau)$ is built up over multiple laser shots (usually $>10^{5}$ ) by recording the photoelectron energy spectrum for a range of delays, $\tau$, between the attosecond pulse and the streaking pulse.

As will be described in section 5, different streaking regimes can be demarcated in terms of the time taken for the photoelectron to escape from the streaking near-field. For gas phase targets the streaking is in the so-called ponderomotive limit-the electron does not experience spatial variations in the streaking near-field. In this regime, energy peaks in the photoelectron spectrum corresponding to different photoemission channels display modulations that follow the vector potential of the streaking field at the time of photoemission. In fact, the streaking trace can be treated as a frequency resolved optical gating (FROG) trace in which the streaking field acts as a pure phase gate function on the photoelectron wavepacket (Itatani et al 2002). FROG is a widelyused technique for characterising femtosecond laser pulses (Trebino et al 1997). The extension of FROG to invert attosecond streaking traces is known as FROG for complete reconstruction of attosecond bursts (FROG-CRAB) (Mairesse and Quéré 2005). It can be performed using iterative algorithms (Kane et al 1997, Kane 1999) initially developed for the inversion of standard laser FROG traces to yield the full electric fields (phase and amplitude) of both the attosecond field and the streaking field. Attosecond streaking was initially used for the temporal characterisation of attosecond pulses. Its ability to fully retrieve the streaking field is particular attractive for the study of nanolocalised plasmonic fields (e.g. surrounding nanoantennas, nanotips and nanospheres) that are excited by ultrafast laser pulses (see sections 3-5). In such studies, it is the plasmonic field that acts as the streaking field. In principle, the time-dependent plasmonic field can be directly compared to the field of the excitation laser pulse by recording a streaking trace in a reference gas-phase atomic target.

\subsection{Case study: Imperial College attosecond beamline}

We now describe the attosecond beamline at Imperial College London (Frank et al 2012) which serves to illustrate the practical implementation of the concepts outlined above and give the reader an idea of the scientific 'tools' required for attosecond physics at the nanoscale. Descriptions of other attosecond beamlines can be found in Fieß et al (2010), Frassetto et al (2014), Huppert et al (2015), Locher et al (2014), Weber et al (2015). The Imperial College beamline employs amplitude gating for the generation of isolated attosecond pulses using the sub-4 fs CEP-stabilised laser system described in section 2.3. It is capable of producing two synchronised attosecond pulses

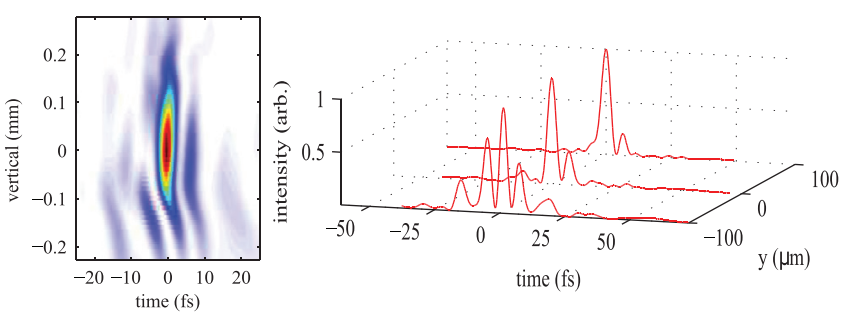

Figure 7. Spatio-temporal characterization of a 1.5-cycle laser pulse.

per laser drive pulse, one in the VUV spectral range $(\approx 20 \mathrm{eV})$ and the other in the XUV range ( $\approx 90 \mathrm{eV})$ (Fabris et al 2015). This setup is targeted at pump-probe studies where both pulses are of attosecond duration, though such schemes are at the limits of current capability due to the limited pulse energy available for the pump step. VUV pulses are advantageous because of the high photo-ionisation cross section of many molecules in this spectral region (Kameta et al 2002) and also because of the higher HHG photon flux possible in this energy range (L'Huillier et al 1991). Attosecond pulses in the $20-40 \mathrm{eV}$ range have previously been generated using polarisation gating (Feng et al 2009, Mashiko et al 2010), though not synchronously with another attosecond pulse. In general, the ability to generate attosecond pulses from the VUV to XUV enables the study of electron dynamics over a wide energy range. This is likely to prove advantageous for the study of nanoscale systems, for example, in photoemission experiments in condensed phase system where electron mean free path is known to be strongly energy-dependent.

Returning to our discussion of the beamline, the $3.5 \mathrm{fs}$, $0.4 \mathrm{~mJ}$ CEP stabilised laser pulses centred at a wavelength of $760 \mathrm{~nm}$ and at $1 \mathrm{kHz}$ repetition rate are introduced into the vacuum beamline through a thin optical window. As shown in figure 8 , they are focused by a concave mirror into two closelyspaced in-line gas jets in which HHG occurs. This common path geometry minimises timing jitter between the pulses (Bothschafter et al 2010, Brizuela et al 2013). The VUV radiation is generated by HHG in a krypton gas target, the XUV radiation in a neon gas target. $\mathrm{Kr}$ proved to be the most efficient rare-gas for VUV harmonic generation. Meanwhile, the higher ionisation potential of $\mathrm{Ne}$ is better suited to XUV generation.

The two pulses propagate collinearly with the NIR laser pulse to a filter assembly comprising different thin foil filters that provide spectral bandpass for the NIR $(7.5 \mu \mathrm{m}$ Kapton foil), VUV (200 nm Sn foil) and XUV (200 nm Zr foil) pulses. By translating the filter assembly across the beam, different combinations of the pulses can be selected. Delay between the collinearly propagating beams is introduced using a two-part MoSi multilayer mirror assembly comprising a piezo-actuated central mirror inside an annular outer mirror (Drescher et al 2001). One beam is reflected by the inner part of the two-part mirror, the other beam by the outer part. To characterise the XUV pulse by attosecond streaking, the XUV and NIR pulses were selected with the appropriate filters and focused by the two-part MoSi mirror into an effusive Ne target. Similarly, for streaking the VUV pulse, the VUV and NIR pulses were selected and focused into an effusive Ar target. Photoelectron 
energies were measured with a time-of-flight (TOF) electron spectrometer (Hemmers et al 1998) with a 0.02 sr collection solid angle and an energy resolution of $\Delta E / E \approx 0.5 \%$. At each delay value, the photoelectron spectrum was integrated for 3 minutes $\left(1.8 \times 10^{5}\right.$ shots at $1 \mathrm{kHz}$ pulse repetition rate $)$.

The streak traces and FROG-CRAB retrievals for the VUV and XUV pulses are shown in figure 9(i) and (ii), respectively. The measured pulse durations were $576 \pm 16$ as for the VUV pulse centred at $20 \mathrm{eV}$ and $257 \pm 21$ as for the XUV pulse centred at $90 \mathrm{eV}$. The error has been deducted from a combination of data analysis error and algorithm error. The former is determined in a bootstrapping kind of way by varying specific parameters in the data analysis routine and observing the final result as a function of those initial filter parameters (see supplemental material in Okell et al (2015)). As for the latter, we have followed the procedure described in Goulielmakis et al (2008). In separate measurements, the VUV pulse energy was determined to be $\approx 0.5 \mathrm{~nJ}$. This should be scalable to higher values by increasing the drive laser pulse energy above the $0.4 \mathrm{~mJ}$ used in this experiment.

\subsection{Attosecond streaking at surfaces-a stepping stone to nanoscale systems}

Attosecond streaking has enabled the complete characterisation of short-wavelength attosecond pulses and has permitted electron dynamics in matter to be resolved with attosecond precision (Drescher et al 2002, Uiberacker et al 2007, Uphues et al 2008, Schultze et al 2010). Progress is being made to widen the scope of attosecond science. A natural extension is the study of condensed phase matter. The response of solids to electromagnetic fields is important in many areas of science and technology. For example, the study of the time evolution of electron-hole pair formation, charge density distributions, and electron propagation in wide-bandgap semiconductors interacting with ultrafast laser fields is of relevance to the development of petahertz signal sampling and processing technologies (Schultze et al 2013, Krausz and Stockman 2014).

In this section we review studies of electron dynamics at solid surfaces using attosecond streaking. This work addresses fundamental questions, such as how is the photoexcited electron affected by the periodic potential as it travels in the solid, and how do other electrons respond in these strongly correlated systems? Laser-assisted photoemission from a surface was first observed in Miaja-Avila et al (2006), where the cross-correlation between a $42 \mathrm{eV}$ XUV pulse and a NIR pulse was measured on a Pt surface. Subsequent condensed-phase attosecond streaking measurements were conducted on single crystal samples of tungsten (Cavalieri et al 2007b) and magnesium (Neppl et al 2012). These works provided the first experimental data on the time delay of photoemission from surfaces more than 100 years after Einstein's paper on the photoelectric effect (Einstein 1905).

In Cavalieri et al (2007b), attosecond streaking on a $\mathrm{W}(110)$ surface was performed using 300 as XUV pulses centred at $91 \mathrm{eV}$, with a 5 fs streaking field (central wavelength of $750 \mathrm{~nm}$ ). To minimise the effect of surface contamination, the measurement chamber was maintained under

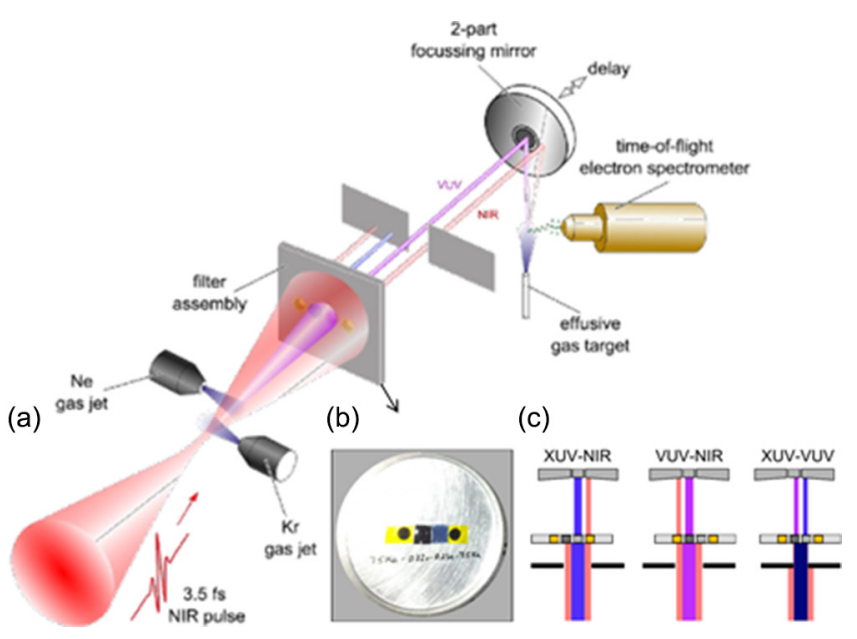

Figure 8. (a) The experimental setup for the generation of synchronised VUV and XUV attosecond pulses by high harmonic generation. NIR laser pulses at $1 \mathrm{kHz}$ repetition-rate are focused into two in-line gas targets of $\mathrm{Kr}$ and $\mathrm{Ne}$ which are independently optimised for efficient VUV and XUV attosecond pulse generation, respectively. The NIR beam and generated radiation travels collinearly to a filter assembly comprising free-standing thin foil filters that allows different combinations of photon energies to be selected. For attosecond streaking measurements, the VUV and NIR beams, or XUV and NIR beams are selected with the appropriate filters and focused into an effusive gas target by a two-part mirror that allows a controllable time-delay to be introduced between the pulses. The photoelectron energy spectrum is measured as a function of delay using a time-of-flight electron spectrometer. (b) A photograph of the filter assembly. From left to right the filters are Kapton (NIR bandpass), indium (XUV bandpass), tin (VUV bandpass) and Kapton. (c) Moving the filter assembly across the beam allows different combinations of the beams to be selected.

ultrahigh vacuum (UHV) conditions. The tungsten crystal was then cleaned before measurements by retracting it to a separate vacuum chamber, where it underwent a number of heating cycles, some of which were in an oxygen environment. The attosecond streaking trace showed two pronounced peaks at $\approx 83 \mathrm{eV}$ and $\approx 56 \mathrm{eV}$ corresponding, respectively, to the $4 \mathrm{f}$-state and valence-band photoemission. By comparing the relative phases of the characteristic streaking oscillations for these two peaks, a delay of $110 \pm 70$ as was found between the emission of photoelectron originating from the localised 4f core states and those liberated from delocalised conduction band states. The relatively large measurement error was reduced in subsequent experiments on W(110) (Neppl 2012) and a smaller delay of $28 \pm 14$ as was found. The larger delay in the initial measurement was attributed to surface impurities, despite the precautions taken.

The origin of this photoemission delay provoked considerable theoretical attraction (Kazansky and Echenique 2009, Zhang et al 2009, Zhang and Thumm 2009, 2011a, 2011b, Krasovskii 2011). Interestingly, no significant delay was found between photoemission from $2 \mathrm{p}$-states and conduction band electrons in attosecond streaking measurements on $\operatorname{Mg}(0001)$ surfaces (Miaja-Avila et al 2006). These measurements used a 435 as pulse at a higher photon energy of $118 \mathrm{eV}$ compared to the tungsten measurements. The quasisynchronous release of the photoelectrons for $\mathrm{Mg}(0001)$ was 


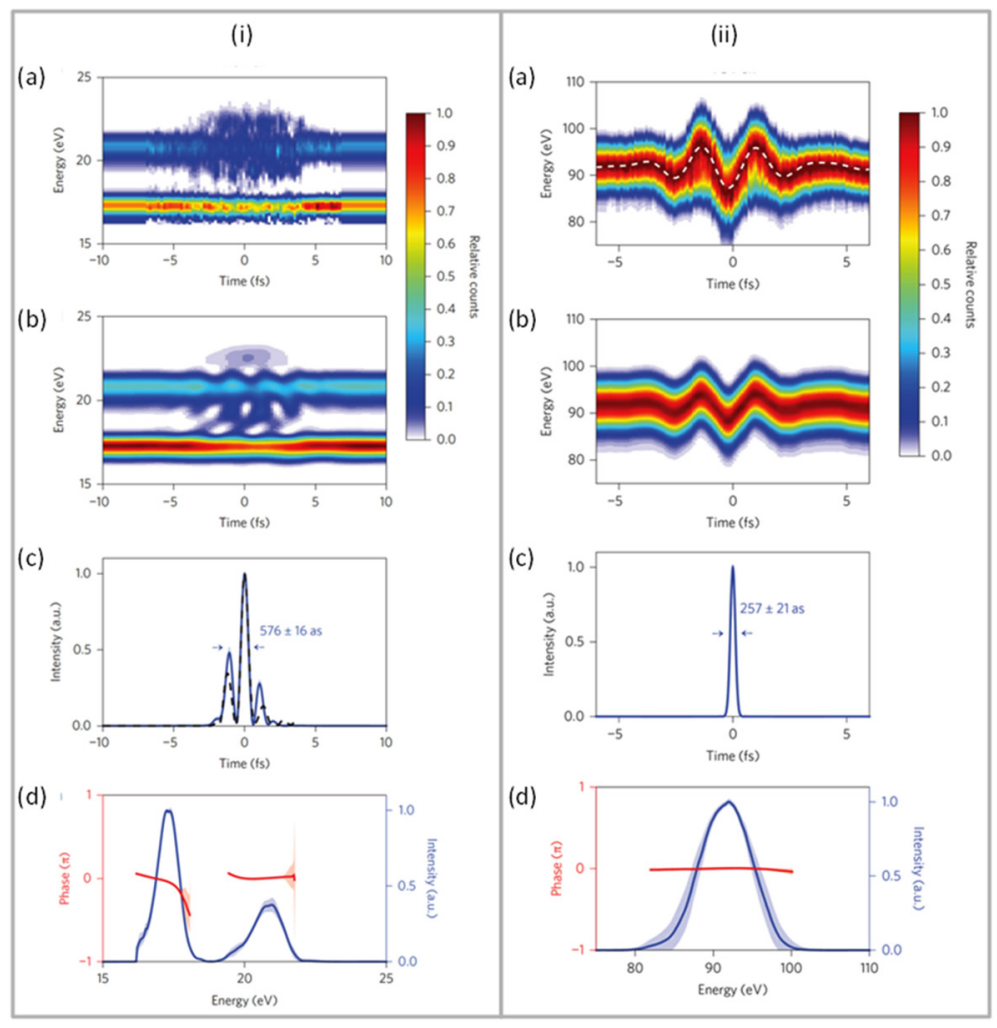

Figure 9. Attosecond streaking measurements of (i) VUV, and (ii) XUV, pulses that were generated synchronously by $\mathrm{HHG}$ in Kr and Ne gas targets, respectively. For both streaking measurements, the other gas target was present. (a) Measured streak trace. (b) Retrieved trace using the FROG-CRAB method. (c) Temporal intensity profile. The VUV intensity profile, (i), exhibits pre and post pulses, as expected due to the transmission of two neighbouring harmonic orders through the Sn spectral filter. The full-width-at-half-maximum VUV and XUV pulse duration were determined to be $576 \pm 16$ as and $257 \pm 21$ as, respectively. (d) Amplitude and phase of the retrieved spectrum. The shaded area represents one standard deviation about the mean.

explained in Miaja-Avila et al (2006) in terms of a simple heuristic model in which the photoemission delay is $\tau_{p}=\lambda_{\mathrm{mfp}} / v_{i}$, where $\lambda_{\text {mfp }}$ is the inelastic electron mean free path in the solid which provides a measure of the average travel distance to the surface, and $v_{i}=\sqrt{2 E_{i} / m_{e}}$ is the initial electron velocity, where $E_{i}$ is the initial electron energy and $m_{e}$ the free electron mass. The electron mean free path was estimated to be $5.9 \AA$ for the $115 \mathrm{eV}$ valence band electrons and $4.8 \AA$ for the $68 \mathrm{eV}$ $2 \mathrm{p}$ electrons. By coincidence, the delay times are thus almost identical for the valence and $2 p$ electrons at $\approx 92$ as.

Further theoretical work, see e.g. Borisov et al (2013), Liao and Thumm (2014), has examined the photoemission delays in tungsten and magnesium using quantum-mechanical models. In Zhang and Thumm (2011b), the role of resonant and nonresonant processes in the origin of the delays is considered. Calculations indicate the valence band electrons can be either retarded or advanced with respect to the localised state electrons, depending on the interplay between the surface

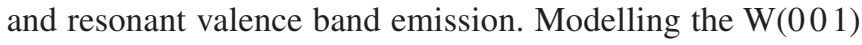
experiment, their calculations revealed a strong surface state contribution $(\tau=0)$ to the valence-band photoemission. Hence, the valence-band photoelectrons appear before the $4 \mathrm{f}$ electrons, with a difference in delay time $\Delta \tau=\tau_{4 f}$. However, for $\operatorname{Mg}(0001)$, resonant processes were calculated to dominate the valence band emission, i.e. bulk-type photoemission, so both $2 \mathrm{f}$ and valence bands are predicted to be photoemitted with delays. This leads to a smaller delay difference-their calculations suggest $\approx 10-20$ as - but still not in agreement with the quasi-synchronous photoemission observed experimentally. In Zhang and Thumm (2011a) the $\operatorname{Mg}(0001)$ experiment was modelled. The relative photoemission delay between the valence band and $2 p$ photoelectrons was found to be sensitive to the electron mean free path and screening of the streaking laser field inside the solid. The quasi-synchronous photoemission was reproduced in these calculations.

In addition to photoemission delays, the temporal structure of the photoemitted electron wavepacket can also be extracted from experimental attosecond streaking traces (Itatani et al 2002). For gas phase atoms, the photoelectron wavepacket can be taken as a 'perfect' replica of the incident XUV pulse (Kienberger et al 2004, Goulielmakis et al 2008). In a solid, be it bulk or nanoscale, extra information is encoded in the temporal properties of the photoelectron wavepacket, for example connected with electron transport, and dispersion. In Okell et al (2015) attosecond streaking measurements were carried out on thin films of polycrystalline $\mathrm{Au}$, a material used widely in plasmonics, and amorphous $\mathrm{WO}_{3}$, a wide-bandgap (3.41 eV) semiconductor (Nakamura and Yamada 1981). These were the first streaking measurements made on solid samples that were not single crystals, and thus they represent a stepping stone towards attosecond streaking of nanoplasmonic fields, as described theoretically in section 5 . 
The experiments were conducted in a UHV $\left(<3 \times 10^{-9}\right.$ mbar) surface-science chamber on the Imperial College attosecond beamline that was described in section 2.6 and 2.7. This chamber contains a similar attosecond streaking set-up to that outlined in section 2.7, comprising a MoSi two-part mirror assembly and the same type of electron TOF spectrometer. Streaking was conducted with a $248 \pm 15$ as XUV pulse centred at $93 \mathrm{eV}$ (pulse duration determined from attosecond streaking in a Ne gas target) and a 3.5 fs NIR streaking pulse which were focused onto the sample as shown in figure 10(i). The NIR intensity on the samples was $10^{10} \mathrm{~W} \mathrm{~cm}^{-2}$, well below the damage threshold of the samples material. At this intensity, above threshold photoemission from the NIR in the valence band region was negligible relative to photoemission from the XUV.

Figure 10(ii) shows the streaking results from a $20 \mathrm{~nm}$ tungsten sample that had been stored at ambient conditions and was not cleaned or prepared in any way prior to the streaking measurements. Separate analysis (XPS, XRD) revealed that the top $9 \mathrm{~nm}$ layer of this sample was amorphous $\mathrm{WO}_{3}$. This is much greater than the electron mean free path in $\mathrm{WO}_{3}$ which was estimated to be $0.5 \mathrm{~nm}$ (Liao and Thumm (2014), Tanuma et al $(2011,2005))$. Hence the measured photoelectron spectra are almost exclusively from the photoemission of $\mathrm{WO}_{3}$. Separate streaking results for a $52 \mathrm{~nm}$ gold film are shown in figure 10(iii). Again, the sample was stored at ambient conditions and no sample cleaning or preparation was carried out. $\mathrm{XRD}$ analysis revealed a polycrystalline surface.

The photoelectron wavepackets were retrieved using FROG-CRAB (see figure 10((ii)c), figure 10((iii)c)). For the WO3 and Au samples the wavepacket durations were measured to be $359_{-25}^{+42}$ as and $319_{-37}^{+43}$ as, respectively. The temporal broadening of the photoelectron wavepackets compared to the XUV pulse duration is $111_{-42}^{+57}$ as and $71_{-54}^{+58}$ as for the $\mathrm{WO}_{3}$ and Au samples, respectively (details on the error analysis can be found in the supplement of Okell et al (2015)). Since the XUV pulse duration was measured independently by attosecond streaking in $\mathrm{Ne}$ atoms, these broadening measurements provide the first direct comparison of the electron wavepacket broadening inherent to photoemission at surfaces versus atomic ionization.

The broadening figures are consistent with a spread in escape times of free-electrons from within a mean free path of the surface (assuming perfect screening of the NIR field at the sample surface), in the spirit of the heuristic model of Miaja-Avila et al (2006). The accuracy of this simple picture of free electron transport in the solid is likely to be a consequence of the XUV photon energy being much larger than the work function of the sample, leading to wavepackets with a free-electron-like character. At lower photon energies, the effective electron mass $m^{*}$ must be considered and the dispersion relation can depart significantly from that of a free electron (Lemell et al 2009). It can exhibit rapid variations in group velocity with energy, which would increase the dispersion broadening of the electron wavepacket as it propagates through the solid (the free-electron group velocity dispersion at $\approx 90 \mathrm{eV}$ accounts for $<1$ as of wavepacket broadening for the $\mathrm{Au}$ and $\mathrm{WO}_{3}$ streaking measurements). Repeating these measurements with lower energy attosecond pulses is therefore an extremely interesting topic for future investigation.

From the streaking traces it was also possible to fully characterize the streaking near-field at the surface of each sample and compare it to the streaking fields retrieved from gas phase streaking measurements in $\mathrm{Ne}$ (see figures 10((ii)d), 10((iii)d)). Though Au has a tendency to form rough surfaces which can enhance the excitation of local surface plasmons (LSPs) and surface plasmon polaritons (SPPs), atomic-force microscopy (AFM) measurement of the gold sample used in this experiment revealed it was mostly plane with a $0.7 \mathrm{~nm}$ rms roughness. Therefore, no substantial plasmonic effects were expected, and indeed, as can be seen in figure 10((iii) d), the retrieved near-field is in close agreement with the field recorded from the gas phase streaking measurements. However, by showing that attosecond streaking is possible on unprepared gold films, these experiments clearly demonstrate that streaking measurements in $\mathrm{Au}$ nanostructures (such as nanoantennas) should, in principle, be able to retrieve plasmonic fields with attosecond precision (see section 5.2). Though such a measurement is likely to pose a significant signal-to-noise challenge, since the nanoplasmonic regions typically make up only a very small fraction of the sample area ionized by the XUV radiation. This may necessitate the use of spatially-resolved electron detection, as discussed in section 5.2. In any case, it may be beneficial to use XUV high photon energies in order to minimise the wavepacket broadening and thus provide the highest temporal resolution possible.

\section{Waveform-controlled imaging of electron photoemission from isolated nanoparticles.}

\subsection{Introduction}

Application of the ultra-short waveform-controlled laser fields to nanostructured materials enables generation of localized near-fields with well-defined field evolution. The optical fields that can be tailored on sub-wavelength spatial and attosecond temporal scales have a high potential for control of ultrafast nonlinear processes at the nanoscale, with important implication for laser driven electron acceleration, XUV generation, and nanoscale electronics operating at optical frequencies. Recently, waveform-controlled enhanced electron acceleration in near-fields was observed in isolated nanoparticles, nanotips, and surface based nanostructures. Here we focus on studies of strong field induced waveform-controlled electron emission from isolated nanoparticles.

\subsection{Imaging of laser-induced electron emission from nanoparticles}

In the experiments exploring the electron emission from isolated nanoparticles, reported in Zherebtsov et al $(2011,2012)$, few-cycle laser fields ( $4 \mathrm{fs}$ at $750 \mathrm{~nm}$ ) have been employed. Such short fields have the advantage that the nanoparticles do not significantly expand during the interaction with the laser 


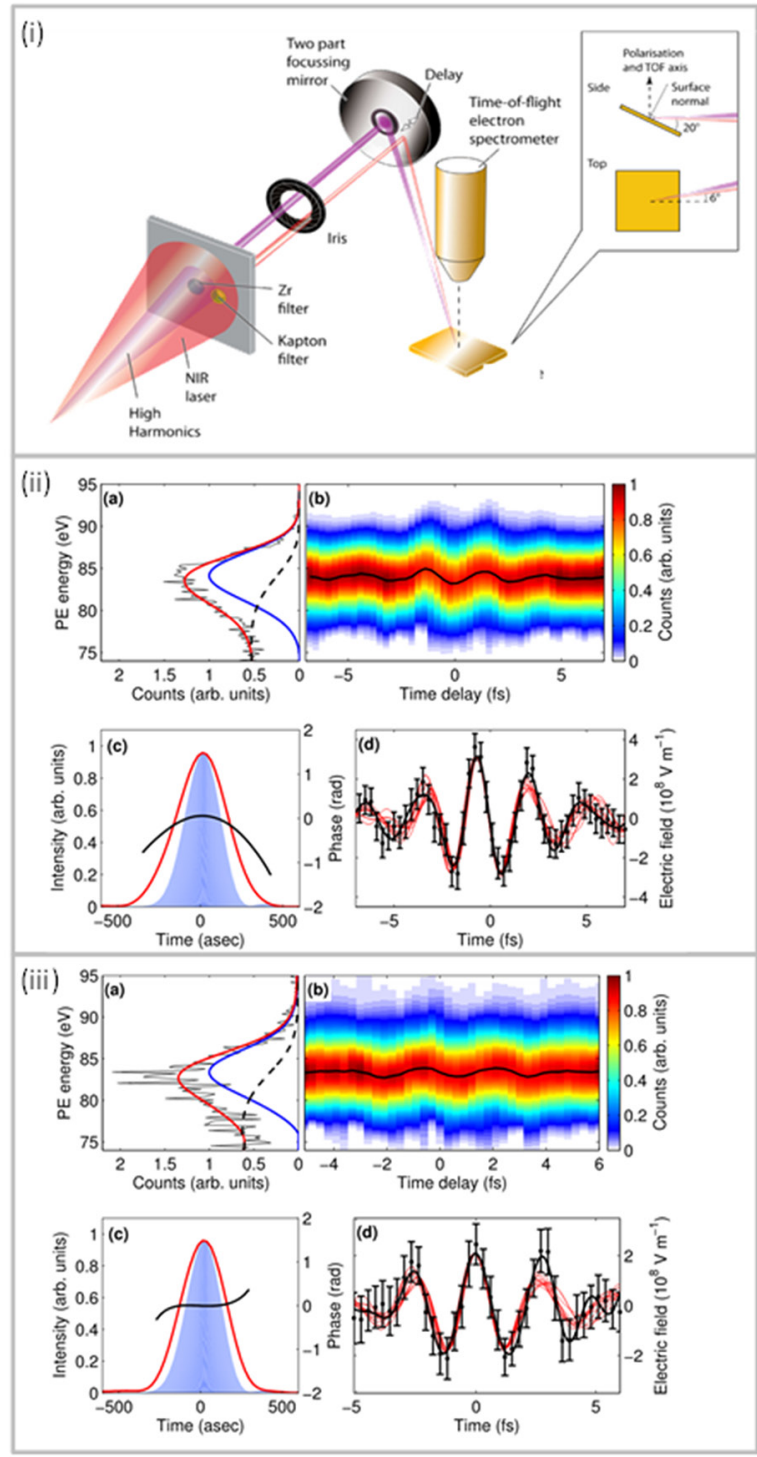

Figure 10. Attosecond streaking at surfaces provides a stepping stone to the nanoscale. (i) Experimental setup for attosecond streaking at surfaces. Few-cycle NIR and attosecond-duration XUV pulses are selected with thin foil filters and focused onto the sample ( $\mathrm{Au}$ or $\mathrm{WO}_{3}$ in this work) using a two-part mirror that provide a variable time delay. An iris allows the NIR intensity to be reduced to a level where the sample if not damaged but there is still sufficient streaking amplitude. Photoemitted electrons are detected with a time-of-flight electron spectrometer. The geometry of the interaction is shown in the inset. The pulses are focused onto the sample with an incidence angle of $20^{\circ}$. The laser polarization lies along the TOF axis. The incident beam is rotated in a horizontal plane by $6^{\circ}$. (ii) Streaking results from an amorphous, $\mathrm{WO}_{3}$ surface. (iii) Streaking results from a polycrystalline Au surface. (a) Valence band photoelectron spectrum with no streaking field: raw data (solid black), Fourier filtered spectrum (red), secondary electron background (dashed black), and background subtracted and filtered spectrum (blue). (b) Streaking trace after Fourier filtering and background subtraction. (c) Photoelectron wavepacket intensity (red) and phase (black) retrieved from streaking trace using FROGCRAB method. (d) Retrieved electric field at surface after bandpass filtering (black curve) and unfiltered data points. For comparison, the retrieved field from eight separate gas phase streaking measurements are shown (red curves). The peak field from each gas phase streak has been scaled to the peak field from the solid sample to aid comparison.

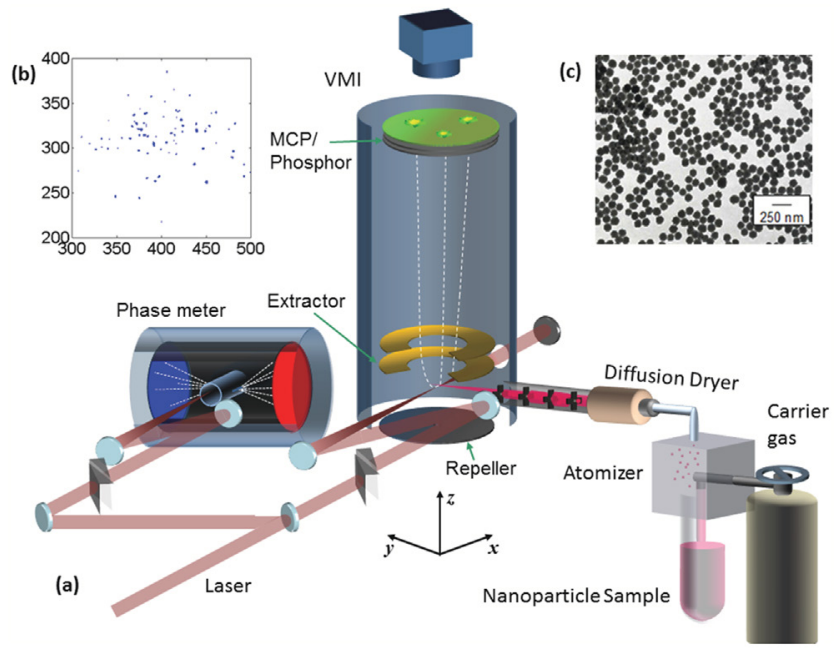

Figure 11. (a) Schematic of the VMI setup with an aerodynamic nanoparticle source and single-shot phase meter. The polarization of the laser was in the plane of the detector. (b) Single shot image recorded by the CMOS camera. (c) TEM image of $95 \mathrm{~nm}$ diameter $\mathrm{SiO}_{2}$ nanoparticles. Figure reused from Zherebtsov et al (2012) (CC BY 3.0).

pulses, and purely electronic dynamics can be investigated. The pulses were generated from the output of an amplified laser system ( $25 \mathrm{fs}$ pulse duration, $790 \mathrm{~nm}$ central wavelength) (Ahmad et al 2009) that was spectrally broadened in a capillary filled with 2.8 bar Ne gas and compressed by a chirped mirror compressor. The CEP of the pulses was measured with a single-shot stereo-ATI phase meter (Wittmann et al 2009, Rathje et al 2012) using a small fraction of the laser beam ( $\sim 15 \%$ ), see figure 11. The main part of the beam was focused into the center of the electrostatic optics of a velocity-map imaging (VMI) setup where it intersected with a nanoparticle beam. The electron emission distribution was projected onto a microchannel plate (MCP)/phosphor screen assembly and light flashes on the phosphor screen were recorded by a highspeed CMOS camera at the full repetition rate of the laser (1 kHz) (Süßmann et al 2011). In order to enable storage of single-shot images at these high rates only pixels with brightness above threshold level were stored on the computer. The single shot detection significantly improved the experimental signal-to-noise ratio as it allows suppressing/identifying background contributions by selecting only the frames that contain the nanoparticle signal.

The $\mathrm{SiO}_{2}$ nanoparticles were prepared by the groups of Graf and Rühl at Freie Universität (FU) Berlin using wet chemistry methods based on the Stöber procedure (Stöber et al 1968) and subsequent seeded growth process. This technique allowed producing particles with diameters in the range $50-550 \mathrm{~nm}$ with a polydispersity of less than $8 \%$ (Zherebtsov et al 2011, Süßmann 2013). After the synthesis the particles were purified by centrifugation/redispersion in ethanol. For size and shape characterization of the samples transmission electron microscopy (TEM) images were taken. Figure 11(c) shows a typical TEM image of the $95 \pm 6 \mathrm{~nm}$ particles. The isolated nanoparticles were delivered into the interaction region by injection of the nanoparticle suspension into a carrier gas and focusing of the nanoparticle stream with an aerodynamic lens. 

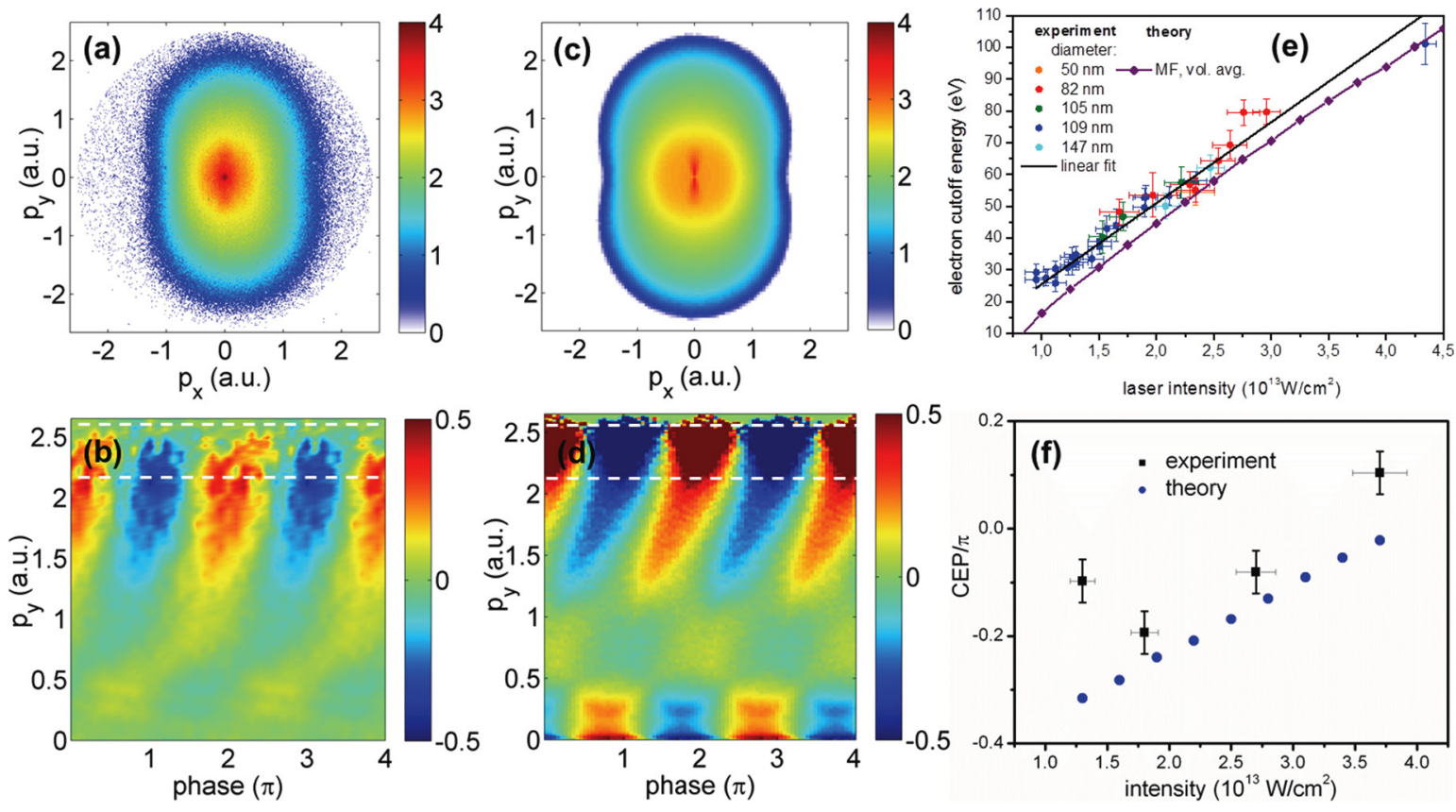

Figure 12. (a) Photoelectron momentum map (projected along $p_{z}$ ) averaged over the CEP (log color scale) and (b) asymmetry of the electron emission as a function of the electron momentum and the CEP measured for $95 \mathrm{~nm} \mathrm{SiO}{ }_{2}$ nanoparticles at $3.7 \times 10^{13} \mathrm{~W} \mathrm{~cm}^{-2}$. (c)-(d) Photoelectron momentum and asymmetry map calculated for the same parameters as in (a)-(b). (e) Intensity dependence of the cutoff in the electron emission from $\mathrm{SiO}_{2}$ nanoparticles with indicated diameters. (f) Dependence of the CEPs at the maximum asymmetry $\phi_{\max }$ of the electron emission from $\mathrm{SiO}_{2}$ nanoparticles of $95 \mathrm{~nm}$ diameter on the laser intensity measured (black boxes) and calculated (blue filled circles). To obtain $\phi_{\max }$ the asymmetry maps were integrated over $p_{y}$ in the cutoff region (indicated by white dashed lines) and fitted with a function $f\left(\phi_{\max }\right)=A \cos \left(\phi_{\mathrm{CEP}}-\phi_{\max }\right)$. Figure reused from Zherebtsov et al (2012) (CC BY 3.0) and with permission from Zherebtsov et al (2011). Copyright 2011 by Macmillan Publishers Ltd: Nature Physics.

\subsection{Waveform controlled electron acceleration in the near- field of a nanosphere}

Figures 12(a)-(d) show typical results from the laser-induced electron emission from $95 \mathrm{~nm}$ diameter $\mathrm{SiO}_{2}$ nanoparticles. The laser polarization is along the $p_{y}$ axis. The electron momentum distribution has an elliptical shape and is elongated along the polarization direction. Few-cycle laser pulses illuminating the nanoparticles offer a possibility to explore the CEP dependence of the electron emission. The CEP dependence of the directional emission can be quantified with an asymmetry parameter

$$
A\left(p_{y}, \phi_{\mathrm{CEP}}\right)=\frac{P_{\mathrm{up}}\left(p_{y}, \phi_{\mathrm{CEP}}\right)-P_{\mathrm{down}}\left(p_{y}, \phi_{\mathrm{CEP}}\right)}{P_{\mathrm{up}}\left(p_{y}, \phi_{\mathrm{CEP}}\right)+P_{\mathrm{down}}\left(p_{y}, \phi_{\mathrm{CEP}}\right)},
$$

where $P_{\text {up }}\left(p_{y}, \phi_{\mathrm{CEP}}\right)$ and $P_{\text {down }}\left(p_{y}, \phi_{\mathrm{CEP}}\right)$ are the angle integrated electron yields (within $\left[-25^{\circ},+25^{\circ}\right]$ angular range) in the up (positive $p_{y}$ momentum) and down (negative $p_{y}$ momentum) directions and $\phi_{\mathrm{CEP}}$ is the CEP. The asymmetry parameter exhibits a pronounced CEP dependence with the largest amplitude near the highest recorded electron momentum. The cutoff of the CEP dependent electron emission is in agreement with the cutoff of the momentum map in figure 12(a) and is at about $50 U_{p}$, where $U_{p}$ is the ponderomotive potential of an electron in the driving laser field. The intensity dependence of the electron emission is illustrated in figures 12(e) and (f). For the studied intensity range $(1-4.5) \times 10^{13} \mathrm{~W} \mathrm{~cm}^{-2}$ the measurements show a nearly linear intensity dependence of the cutoff energy with an average scaled cutoff of about $53.0 U_{p}$.
The obtained cutoff is much higher than the modified classical atomic cutoff of $\sim 24 U_{p}$ that is expected for the dielectrically enhanced field near a nanosphere. The maximum asymmetry phase $\phi_{\max }$ increases with the laser intensity (except at the lowest intensity point).

The mechanism of the enhanced electron acceleration was analyzed with quasi-classical trajectory-based simulations using the mean-field-Mie-Monte-Carlo $\left(\mathrm{M}^{3} \mathrm{C}\right)$ model (Zherebtsov et al 2012). Results of these calculations performed for the same parameters as in the experiment are presented in figures 12(c)-(f). The simulations reproduce the main features of the experiment such as the overall shape of the momentum and asymmetry maps as well as the cutoff value. The simulation shows a similar increase of $\phi_{\max }$ with laser intensity as the experiment except for the lowest intensity point (figure 12(f)). The discrepancy at the lowest intensity may be ascribed to a deviation of the initial ionization mechanism from the pure tunneling regime assumed in the model.

\subsection{Effect of near-field deformation on the electron photoemission from a nanosphere}

The angle resolution provided by VMI detection offers a possibility for a more detailed visualization of the CEP dependent photoemission. It was demonstrated recently that phase controlled electron photoemission provides a sensitive probe for localized fields (Süßmann et al 2015, Seiffert et al 2016). In these contributions isolated nanospheres served as a test 

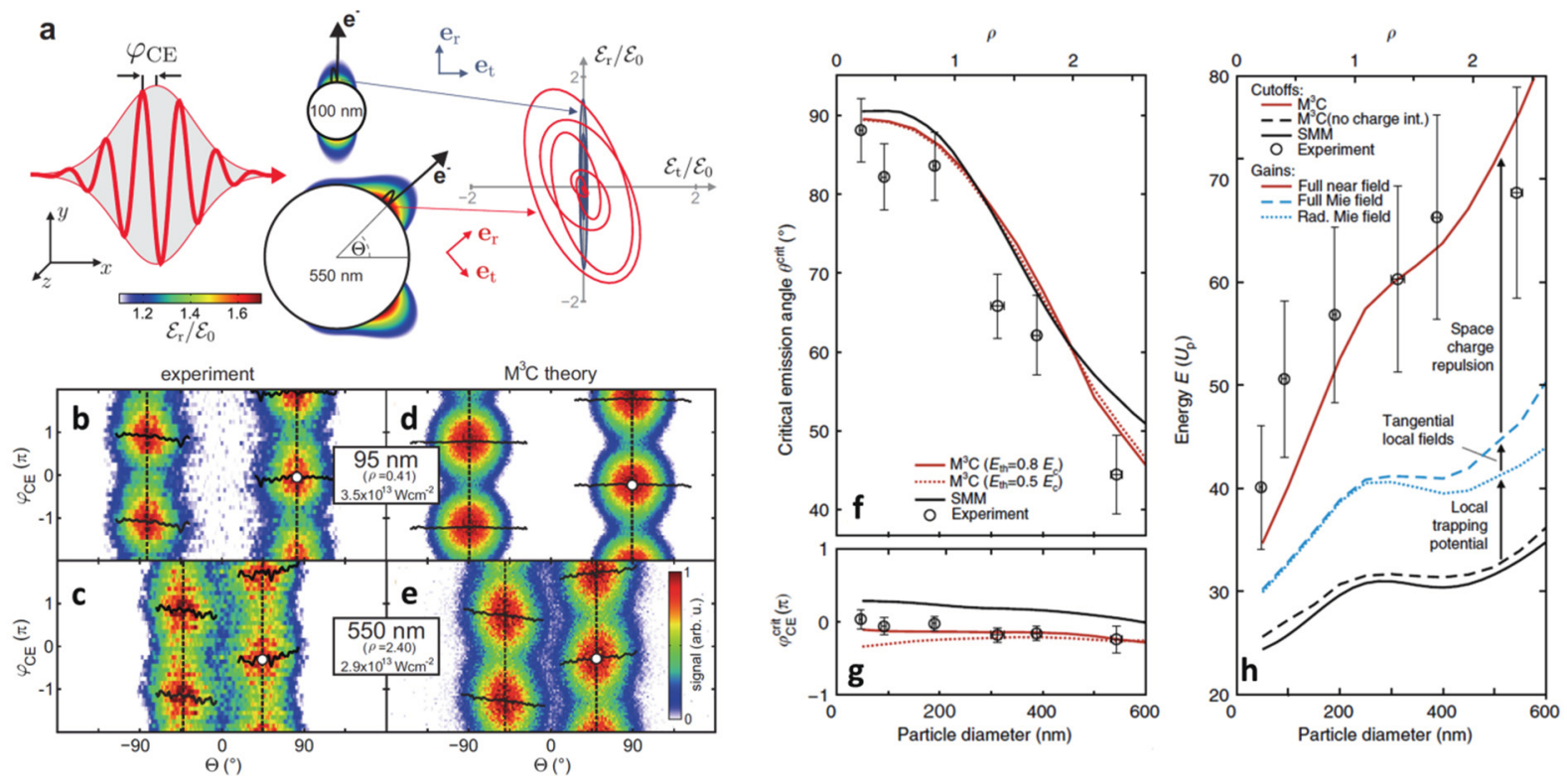

Figure 13. (a) Peak radial field enhancement in the $x-y$ plane at $z=0$ obtained by Mie solution for $\mathrm{SiO}_{2}$ spheres illuminated with a 4 fs linear polarized laser pulse centered at $720 \mathrm{~nm}$ and a CEP of $\phi_{\mathrm{CEP}}=0$ (left). Field evolution in the local reference frame in the points of maximum field enhancement (right). (b)-(e) Measured (b)-(c) and simulated (d)-(e) angle and CEP-resolved electron yields of energetic near-cutoff electrons. The white dots indicate CEP values $\phi_{\mathrm{CEP}}^{\text {crit }}$ and emission angles $\theta^{\text {crit }}$ of maximum upward emission. (f)-(h) Particle size dependence of the critical emission angle (f), critical phase (g), and cutoff energies (h). The symbols and lines indicate measured and calculated parameters. The simple man's model (SMM) simulations are described in detail in Seiffert et al (2016), Süßmann et al (2015). Figures reused from Seiffert et al (2016) (CC BY 3.0), Süßmann et al (2015) (with permission from Macmillan Publishers Ltd: Nature Communications, Copyright (2015).

system for the generation of near-fields with adjustable polarization and spatial characteristics. Figure 13(a) shows the field enhancement distribution of the radial electric field as predicted by Mie theory for 100 and $550 \mathrm{~nm}$ diameter $\mathrm{SiO}_{2}$ particles. For the particle much smaller than the wavelength of the incident field the near-field exhibits dipolar character and peaks along the laser polarization axis (see section 2.1). As the particle size becomes comparable to the laser wavelength the effect of the field propagation starts to play a significant role. This results in a shift of the maximal enhancement region from the propagation direction towards the rear side of the sphere and a nontrivial elliptical near-field shape.

The effect of field propagation on the phase controlled electron photoemission is illustrated in figures 13(b)-(e). For small nanospheres the electron yield peaks at the poles of the particle with the maximum signal at a critical CEP, $\phi_{\text {CEP. }}^{\text {crit }}$ For the large particles the electron yield shows similar phase dynamics and a significant shift of the critical emission angle to almost $45^{\circ}$. The size dependence of the main emission parameters is illustrated in figures $13(\mathrm{f})-(\mathrm{h})$. The model simulations show that the electron acceleration process is dominated by the radial component of the near-field with the tangential component starting playing role only for nanoparticles larger than $\sim 300 \mathrm{~nm}$ diameter (figure 13(h)). The polarization of the near-field seems to have only limited effect on the CEP dependence of the electron emission (figure $13(\mathrm{~g})$ ). The experiment shows good agreement with the $\mathrm{M}^{3} \mathrm{C}$ simulations, supporting proper description of a tunable directionality and attosecond control of electron dynamics in strongly deformed near-fields. Quantitative analysis of different many-particle contributions to the acceleration process (figure 13(h)) shows that the local trapping potential is only weakly size-dependent. That can be explained by the local character of this potential, which is mainly determined by the local electron density. On the other hand, the contribution from the space-charge repulsion within the escaping electron bunch increases strongly with the particle size indicating its sensitivity to the full electron distribution.

The trajectory based model allows correlation analysis between the electron emission position and its final momentum direction. Figure 14(a) illustrates two limiting cases of the emission from a sphere. The radial emission allows correlation of the final momentum direction to the initial birth angle. The analysis of energetic trajectories from small nanospheres, where the tangential field component is negligible, shows transition from unidirectional to radial emission with increase of the laser intensity (figures 14(b) and (c)). This intensity dependence can be attributed to the effect of the trapping potential that favors radial emission. For large nanoparticles the tangential and normal components of the driving field at the surface of the particle become comparable (figure 13(a)). The non-diagonal shape of the correlation plots reflects the increased importance of the tangential field component for the acceleration process.

\section{Attosecond control of electrons at nanoscale needle tips}

As discussed in section 1, hallmarks of attosecond physics include electric-field driven control of electron motion and the re-scattering plateau. First observed and understood in the context of atomic physics in the gas phase in the 1980s and 1990s 

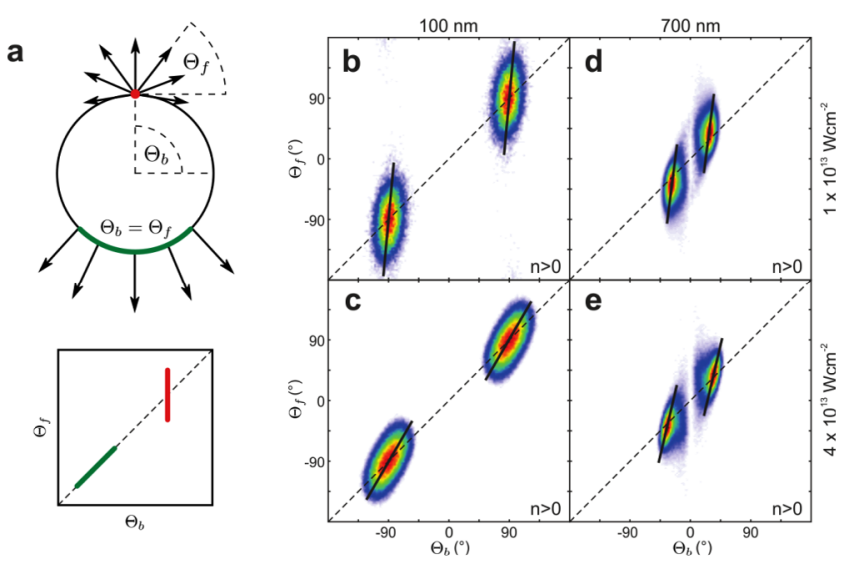

Figure 14. (a) Schematic representation of correlation characteristics between birth angle $\Theta_{b}$ and final angle $\Theta_{f}$ of the unidirectional (red) and radial (green) emission. The birth and final angle are defined as projections of birth position and final momentum vector on the $x-y$ and $p_{x}-p_{y}$ planes respectively. (b)-(e) Correlation plots for energetic electrons $\left(E>E_{c} / 2\right)$ emitted from large and small nanospheres at two different intensities. Here $E_{c}$ denotes the cutoff energy of the electron emission. Only trajectories with electron collisions $(n>0)$ were selected for the analysis. The dashed black line represents the case of radial emission. Figure reused from Seiffert et al (2016) (CC BY 3.0).

(see e.g. Krausz and Ivanov (2009), Milošević et al (2006), Scrinzi et al (2006) and references therein), at solids and in particular at nanostructures they have been first observed and theoretically understood about two decades later (Krüger et al 2011, Krüger et al 2012b, Herink et al 2012, Wachter et al 2012, Piglosiewicz et al 2014). Attosecond physics phenomena at single nanostructures have been discussed in several original papers and review articles (see, e.g. Hommelhoff and Kling (2015)), which is why here we only give a comprehensive overview of the field and refer the reader interested in the technical details to the more extensive review articles and original papers.

About 50 years ago in his seminal work Keldysh has come up with a theory that insightfully connects atomic tunnelling ionization in a strong laser field with ionization in a static electric field (Keldysh 1965) (see section 1.1). The same relation holds for solid surfaces, not discussed in Keldysh's pioneering work: DC field emission from solids (see Fursey (2005) and references therein) and optical tunnelling photoemission are closely linked, which is why the latter is consequently called optical field emission (Bunkin and Fedorov 1965). In addition, multiphoton emission, another limiting case of the Keldysh theory, may also arise at solids. DC field emission routinely requires sharp nanoscale needle tips in order to reach field strengths on the order of $1 \mathrm{~V} \mathrm{~nm}^{-1}$ on the tip surface by the virtue of the lightning rod effect. The optical counterpart of this DC field enhancement effect at nanotips, optical nearfield enhancement, pushes laser-tip interactions into regimes of high intensity of up $10^{14} \mathrm{~W} \mathrm{~cm}^{-2}$, corresponding to peak electric field strengths of $2.7 \mathrm{~V}^{-1}$. This is of great practical relevance: it enables strong field physics experiments without the use of amplified laser systems. Initial studies focusing on the very nature of femtosecond laser-induced electron emission pointed to tunnelling photoemission (Hommelhoff et al 2006b,

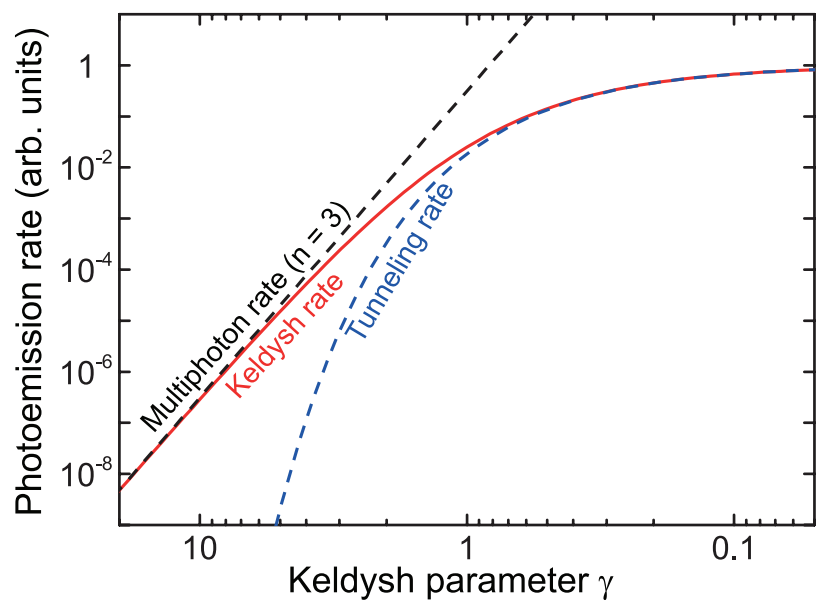

Figure 15. Illustration of the transition from multiphoton to tunnelling regime. Photoemission rate as a function of Keldysh parameter $\gamma$ (Tóth et al 1991, Keldysh 1965) (red full curve). The soft kink indicates the transition from multiphoton limit (dashed black line) to the tunnelling limit (dashed blue curve). In this calculation, the workfunction is $4.5 \mathrm{eV}$ and the photon energy $1.5 \mathrm{eV}$. The theory curves are calculated with exponential accuracy.

2006c) or to multiphoton photoemission (Barwick et al 2007, Ropers et al 2007a, 2007b, Hilbert et al 2009), the two limiting regimes for oscillating fields of the Keldysh theory. Spectrally resolved measurement demonstrated above-threshold photoemission (ATP), the analogue of gas-phase ATI, and a clear ponderomotive shift of above-threshold peaks-hallmarks of the onset of a strong field photoemission regime (Schenk et al 2010).

The transition from the multiphoton regime (Keldysh parameter $\gamma \gg 1)$ to the tunnelling regime $(\gamma \ll 1)$ was first reported in photoemission from a gold nanotip in a nearinfrared laser field (Bormann et al 2010). Similar to the initial work performed at a planar solid surface (Tóth et al 1991), the authors observed a soft kink in the scaling of photocurrent with intensity. At low intensity, the multiphoton scaling dominates, with the current $j$ scaling as $j \propto I^{p}$, with $I$ the laser intensity and $p$ the minimum required number of photons for photoemission. Around an intensity corresponding to the intermediate regime of Keldysh parameter $\gamma \sim 1$, the scaling changes into a field-dependent tunnelling behavior $(j \propto \exp -C / \sqrt{I})$, featuring a much less steep slope (see figure 15 for an illustration). This transition has been observed in many more experiments for different wavelengths and materials, including plasmonic nanostructures and films (see, e.g. Dombi et al (2010), Keathley et al (2013), Piglosiewicz et al (2014), Teichmann et al (2015), and can be well modeled by strong field theory (Yalunin et al 2011). The change of slope as a function of intensity appears to be more rapid than expected from this theory, which has been explained with an additional photocurrent contribution from the laser field penetrating into the metal surface (Bormann et al 2010). Also strong saturation of the photoemission yield at intensities slightly higher than the kink has been reported (Piglosiewicz et al 2014).

Tunnelling photoemission is prompt by definition and features sub-optical-cycle-resolved bursts of electron 
wavepackets (Hommelhoff et al 2006b, Yalunin et al 2011, Krüger et al 2012a), even in the intermediate, non-adiabatic tunnelling regime around $\gamma \sim 1$ (Yudin and Ivanov 2001). Strongly delayed photoemission, on the other hand, is a sign of the formation of an excited non-equilibrium electron distribution inside the solid. A prominent example is thermally enhanced field emission where the laser pulses heat the electron gas (Kealhofer et al 2012). Pronounced electron-electron and electron-phonon scattering can also result in a photoemission delay, as encountered in a comparatively long laser pulse (Yanagisawa et al 2011) or, as it has been argued, when electrons return to the surface and undergo backscattering inside the metal (Yanagisawa et al 2016). While the first experiments resorted to verify prompt photoemission by measuring current additivity in an autocorrelation experiment (Hommelhoff et al 2006b, Ropers et al 2007a, Hilbert et al 2009), a recent study indicates that photoemission can be prompt up to a Keldysh parameter of $\sim 13$ (Juffmann et al 2015). This study employed a microwave cavity to streak photoelectrons from a nanotip, measuring their emission time with an accuracy of about 2 fs. If identified, delays smaller than 2 fs already are on a suboptical-cycle level and might be interpreted with tunnelling time delays, a research subject of high current interest.

As outlined above, the regimes of atomic gas-phase ionization can readily be transferred to photoemission from solid surfaces. This holds especially true for the electron dynamics following photoemission, as revealed by spectral features. The re-scattering plateau, its cutoff and a comparison to early work in atoms is shown in figure 16 (Lindner et al 2005, Krüger et al 2011). Clearly, the overall shape of the spectra is very similar, with the direct part (exponential decrease of the count rate at small energies), the plateau part, which is terminated by the cutoff and a subsequent steep decrease in count rate. Both spectra exhibit peaks spaced by the photon energy, which is a clear sign of ATI and ATP. Because of the almost identical driver wavelengths of around $800 \mathrm{~nm}$ in the two experiments, the photon energies are about equal, indicating that although the shape of the spectra are very similar, the energy scales differ. This is owed to the fact that the intensity driving electron re-scattering is very different (gas: $7 \times 10^{13} \mathrm{~W} \mathrm{~cm}^{-2}$, tip: $4 \times 10^{11} \mathrm{~W} \mathrm{~cm}^{-2}$ in the bare focus). This large discrepancy is partially lifted by optical near-field enhancement at the nanoscale needle tip, leading to an effective intensity of $\sim 1.5 \times 10^{13} \mathrm{~W} \mathrm{~cm}^{-2}$ at the tip's apex. In addition, the ionization potential (or workfunction in metals) is different: in xenon, the ionization potential is $12.1 \mathrm{eV}$, while in tungsten it is $4.5 \mathrm{eV}$.

Accordingly, CEP resolved spectra of argon gas and tip look very similar, see figure 17 . The similarity of these data is based in the understanding that both the electron emission mechanism as well as the external dynamics of the electron in the laser field are essentially identical. The Keldysh parameter in both cases leans towards optical-cycle resolved electron emission in the non-adiabatic tunnelling regime (Yudin and Ivanov 2001), after which the dynamics of the electron in the field of the laser, including the re-collision process of the electron with the parent matter, seems identical.

For the general understanding of the process, the atomic physics picture view holds: Corkum's seminal three-step
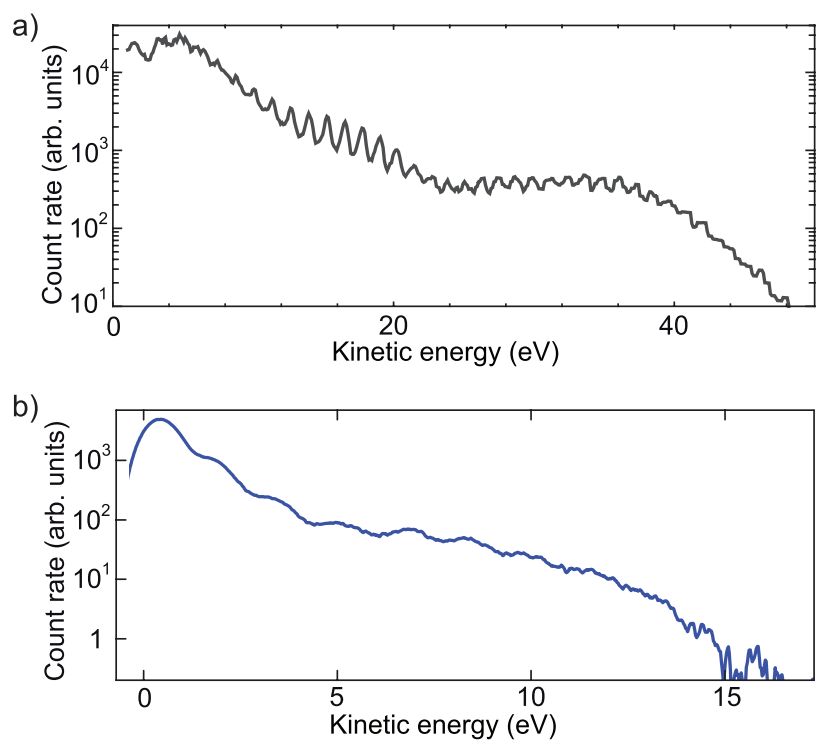

Figure 16. For comparison: photon-order resolved strong field spectra from xenon atoms (a) and from a tungsten needle tip (b). In both cases, the plateau and the cutoff are clearly discernible. Figure in (a) reused with permission from Paulus et al (2004) and modified. Copyright 2004 by IOP Publishing.

model (Corkum 1993) fully applies and can explain the position of the cutoff, while the matter wave interference picture explains the photon orders, and their nonappearance for certain CEP values, provided that few-cycle laser pulses are used: Photon orders may not show up if electrons can only be accelerated to high enough energies during a single laser cycle. In that case, no other time-window exists from which electrons with sufficient energies can be released in order to interfere with those ionized by the first optical cycle (Krüger et al 2011, Krüger et al 2012a). Intriguingly, a very simple model based on the propagation of Gaussian electron pulses suffices to explain the spectra, notably over the full dynamic range of several orders of magnitude in count rate (Krüger et al 2012b). Because the solid surface breaks the symmetry, the number of electron trajectories that contribute are only half as many as in the case of atoms in the gas phase, making the system even simpler to describe. Hence it may be called a model system for strong field processes at surfaces. We conclude that it is predominantly the dynamics of the laser-driven single free active electron that determines the shape of the spectra, in particular the plateau and cutoff regions. The direct part and its behavior can be well modeled with extant theory such as PPT and ADK (Perelomov et al 1966, Ammosov et al 1986, Yalunin et al 2013, Bionta et al 2014). When, however, many photoelectrons are emitted per laser pulse and influence each other by Coulomb repulsion, the simple picture of direct photoemission and re-scattering might not be sufficient anymore, as a recent study suggests (Yanagisawa et al 2016). Here, the Coulomb repulsion itself leads to the formation of a plateau in electron spectra, whereas the low-energy part is formed by electrons that have been slowed down by scattering effects.

With this understanding, we can turn the perspective around and utilize the single active electron as a probe to measure the strong field that is driving it, namely the optical 

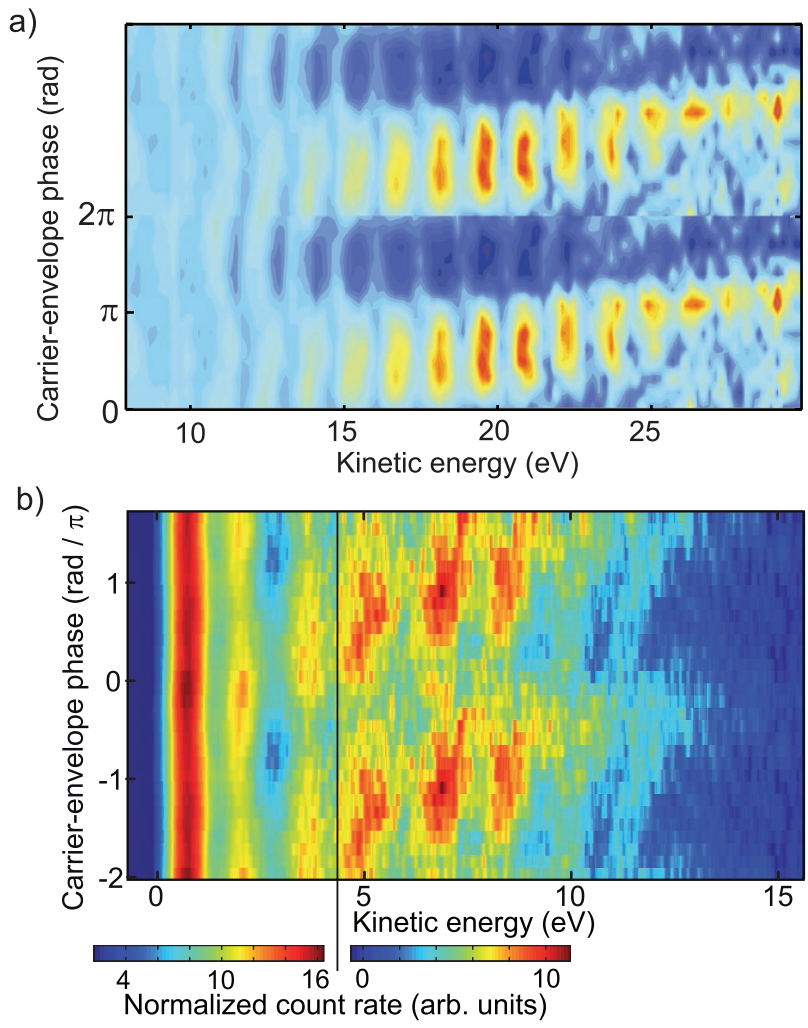

Figure 17. CEP-resolved spectra recorded in atomic argon gas (a) and at a tungsten tip (b). In both cases only the range $0 \ldots 2 \pi$ was measured. The same data is shown twice on top of each other for clarity. Figure (a) is reused with permission from Lindner et al (2005) and (b) from Krüger et al (2011). Copyright 2005 by American Physical Society and 2011 by Macmillan Publishers Ltd: Nature, respectively.

near-field at the nanostructure. Optical near-fields decay over a characteristic length not given by the driving wavelength, but given by the typical dimension of the structure provided the latter is much smaller than the former (Novotny and van Hulst 2011, Novotny and Hecht 2012). Typically, the sharpest nanostructures have kinks and edges with radii of curvature larger than $3 \mathrm{~nm}$, hence the decay length of the near-field is usually larger than $\sim 2 \mathrm{~nm}$ (Thomas et al 2013). With electrons driven by $800 \mathrm{~nm}$ laser light and effective field strengths in the range of $1 \mathrm{~V}^{-1}$, the classical excursion length according to the three-step model equals $0.3 \mathrm{~nm}$. Therefore, in the re-collision scenario the electron only samples a field region extending the excursion length away from the nanostructure surface. For such small excursion lengths, the near-field can be considered constant for all but the sharpest nanostructures. Based on this idea, the field enhancement factor can be accurately measured, as has been done in Krüger et al (2014), Thomas et al (2013). Similarly, electron acceleration at arrays of gold nanostructures (Dombi et al 2013) and nanopillars (Nagel et al 2013) was used to deduce the magnitude of the field enhancement, demonstrating how their shape affects the behaviour of optical near-fields through plasmon resonances. Also for non-plasmonic materials a strong shape dependence is expected that can lead to a dramatic increase of field enhancement (Thomas et al 2015).
Recent progress in needle tip-based optical cycle resolved physics experiments encompasses work at longer wavelengths than the typical $800 \mathrm{~nm}$ of the initial work, extending up to 8 $\mu \mathrm{m}$ (Herink et al 2012, Park et al 2012, 2013, Yalunin et al 2013, Piglosiewicz et al 2014). Longer wavelengths are of interest for several reasons. First, the typical time scales are prolonged, meaning that the tunnel barrier responsible for electron emission is established for longer times. Thereby, it is easy to reach deep into the tunnel regime (note the definition of the Keldysh parameter in terms of tunnel duration, see section 1.1). Second, by a similar token, the electron, spending more time within a single laser cycle, is accelerated to larger kinetic energies, which could be of interest for source applications and the like. However, these are limited by the third point, namely that the increased classical excursion length of the classical electron trajectory can now easily overcome the optical field's decay length, which, as pointed out above, is given by the dimensions of the nanostructure and not the driving wavelength. The last point is closely related to the discussion of attosecond physics in inhomogeneous fields, treated in section 8.5 and 9.4. The observations reported in the aforementioned experimental works confirmed two outcomes of these effects, namely the suppression of re-scattering and electron emission followed by instantaneous acceleration within less than an optical cycle. Like in the near-infrared case (Krüger et al 2011), the electron motion can be controlled with the CEP (Piglosiewicz et al 2014) and the nearfield can be investigated based on electron kinematics (Park et al 2013). Wavelength-scaling studies recently included also the terahertz regime, which was explored as a means to streak photoemission (Wimmer et al 2014) or drive field emission (Herink et al 2014).

Until recently, standard materials such as tungsten and gold have been used in the study of femtosecond laser driven electron emission from needle tips. It has been pointed out that this was not a bad choice, as the comparably large heat conductivity of both materials seems central to observing prompt electron emission mechanisms, such as multiphoton and tunneling processes (Kealhofer et al 2012). Nanotips made from highly doped silicon were also proven to support these processes (Swanwick et al 2014). In contrast, hafnium carbide, a material with an extremely high melting point of $\sim 4200 \mathrm{~K}$, which thus may also seem well suited as a prompt femtosecond electron emitter, displays a large thermal and thus noninstantaneous electron emission current contribution, due mainly to its poor heat conductivity (Kealhofer et al 2012). It will be interesting to see if strong field effects can be observed at extremely well controlled and rugged modern materials, such as carbon nanotube electron emitters (Bionta et al 2015).

Based on the fundamental understanding gained in the last decade, the research field has enabled a range of applications of laser-driven nanotip photoemission, in particular as a source of ultrashort electron pulses. Crucial is the development and characterization of various electron source designs, a very active research area of recent years (Paarmann et al 2012, Lüneburg et al 2013, Hoffrogge et al 2014, Bormann et al 2015, Ehberger et al 2015, Schröder et al 2015a, Vogelsang et al 2015, Müller et al 2016). The spectrum of applications 
ranges from demonstrations of quantum optical phenomena with free electrons (Caprez et al 2007, Feist et al 2015) via the generation of x-ray pulses (Foreman et al 2013) to ultrafast microscopy and low-energy electron diffraction experiments for fundamental material science (Quinonez et al 2013, Gulde et al 2014, Müller et al 2015). Notable is also the source development focussing on emitter arrays for, e.g. injecting high-brightness electron beams into accelerators (Ganter et al 2008, Tsujino et al 2008, 2009, Mustonen et al 2011, 2012, Keathley et al 2013, Hobbs et al 2014, Swanwick et al 2014).

However, none of these applications so far goes beyond the femtosecond regime and makes direct use of the sub-opticalcycle nature of strong field photoemission or the electric field control of electron motion. Due to the matter-wave dispersion of free electrons, attosecond dynamics are essentially limited to the vicinity of the nanostructure. Apart from probing the near-field and its structure as described above, attosecond control capabilities have been explored for detecting the CEP of few-cycle laser pulses (Hommelhoff et al 2006a, 2006b, Krüger et al 2011, Schenk et al 2011, Piglosiewicz et al 2014). Experimental efforts are currently underway towards attosecond electronics where the electric field switches and controls current between nanoelectrodes. Here, in an initial experiment the operation of a nanoscale vacuum tube diode in the femtosecond regime was reported, with the prospect of extending this time scale to the sub-optical-cycle regime (Higuchi et al 2015). Also it will be interesting to see if HHG-the recollision mechanism of attosecond science per excellence-can be observed, without the use of an additional gas, and controlled by the CEP (Ciappina et al 2014c). Last, we note that the physics discussed here is also of current interest in the context of nanoplasmonics (Dombi et al 2013, Schertz et al 2012, Kusa et al 2015), optical control of photoemission sites on a nanostructure (Yanagisawa et al 2009, 2010) and VMI (Bainbridge and Bryan 2014).

\section{Attosecond streaking in nanolocalized plasmonic fields}

While the waveform controlled electron emission contains some spatial information about the near-field distribution (Krüger et al 2011), reconstruction of the time evolution relies to a large extent on model calculations. A pump-probe approach provides more direct access to the time-resolved near-field dynamics. So far, ultrafast plasmonic near-fields surrounding nanowires, nanoantennas, and nanotips have been fully characterized using femtosecond pulse characterization techniques (Hanke et al 2009, Rewitz et al 2012, Anderson et al 2010, Dombi et al 2010, Vogelsang et al 2015). Attosecond streaking measurements are expected to yield an even deeper understanding of the collective electron dynamics governing plasmon formation and decay, where transport and interaction effects on sub-cycle timescales are expected to be important.

As mentioned previously in section 2.5, attosecond streaking measurements can be used to trace attosecond electron dynamics in gas-phase samples and plain solid surfaces, a
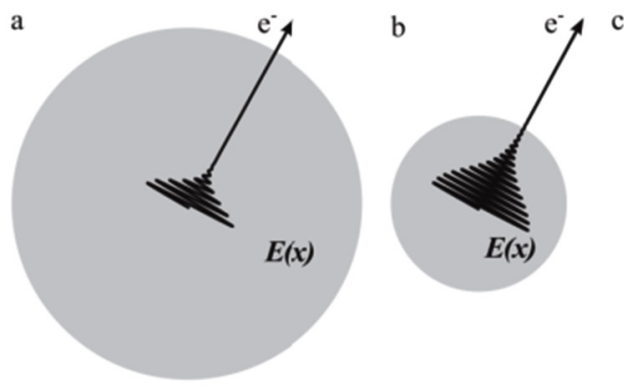

$\mathrm{E}(\mathrm{x})$

$\mathrm{E}(\mathrm{x})$

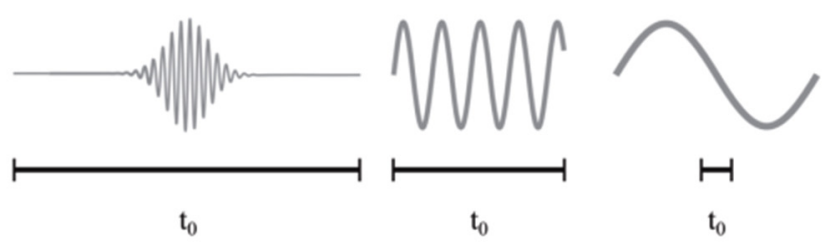

Figure 18. Schematic illustration of the three attosecond streaking regimes (see text for details). Figure reused from Kelkensberg et al (2012) (CC BY 3.0).

and to fully characterize both the near-infrared (NIR) laser pulses (the streaking field) and the extreme ultraviolet (XUV) attosecond pulses. In attosecond streaking measurements on plasmonic nanostructures, the streaking field is replaced by a plasmonic field excited by (and typically enhanced with respect to) the incident NIR laser field, while the XUV acts as a probe by photoemitting an electron wavepacket that subsequently gets accelerated (streaked) in the plasmonic field (Stockman et al 2007). In principle, similar information to standard streaking measurements can be obtained: the temporal structures of the streaking field and the XUV pulse, and information about attosecond electron dynamics taking place in the system. For plasmonic nanostructures, however, the situation is much more complex than in standard streaking measurements because the nanolocalized fields are spatially inhomogeneous (Stockman et al 2007). The shift of the XUV photoemission is determined by the external field

$$
v_{f}\left(t_{e}\right)=v_{0}-\int_{t_{e}}^{\infty} \frac{e E(\mathbf{r}, t)}{m} \mathrm{~d} t,
$$

where the field $E(\mathbf{r}, t)$ has spatial and temporal dependence. Figure 18 illustrates three different regimes of streaking in spatially inhomogeneous fields as reported in Kelkensberg et al (2012), Skopalová et al (2011), Stockman et al (2007). In the ponderomotive limit the streaking field pulse duration $t_{p}$ is much shorter than the time it takes the electron to leave the near-field $t_{0}\left(t_{p} \ll t_{0}\right)$ and the electron does not experience spatial variation of the near-field (figure 18(a)). This corresponds to the case of conventional streaking in gas targets. Figure 18(c) illustrates the other, instantaneous limit when the electron leaves the localized field within a fraction of the optical cycle $T\left(t_{0} \ll T\right)$. This corresponds to quasi-electrostatic acceleration and the streaking field can be described by an electrostatic scalar potential. In contrast to conventional, ponderomotive streaking, in the instantaneous regime the electron streaking curve follows the electric field evolution. Finally, in the intermediate regime the electron experiences the field 

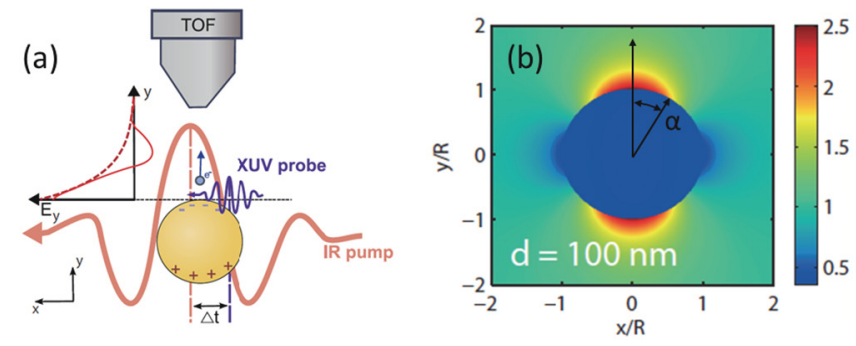

Figure 19. Schematic of attosecond streaking on an isolated nanosphere (a). Amplitude of the field $\left(E_{y}\right)$ distribution at an $\mathrm{Au}$ sphere of $100 \mathrm{~nm}$ diameter illuminated at $720 \mathrm{~nm}$ (b). The field is normalized to the incident field. Figure reused with permission from Süßmann and Kling (2011b). Copyright 2011 by American Physical Society.

during several optical oscillations $t_{0} \approx T$ (figure 18(b)) and the streaking trace shows a phase-shift, which lies in-between the other two limits. Since the retrieval of the near-field in this case requires extensive modeling, the other two regimes are most desirable.

\subsection{Attosecond streaking from an isolated nanosphere}

Due to their simple shape and the possibility of an analytical description of the near-field of isolated nanospheres, they can be used as a reference system for tracing plasmonic excitations (see section 2.1). Isolated nanoparticles of well-defined size and shape can be produced by wet chemistry methods (Stöber et al 1968, Sau and Murphy 2004) and introduced into the interaction region by employing aerodynamic lenses (Zherebtsov et al 2011, 2012) or optical trapping (Hansen et al 2005). Figure 19(a) shows a schematic of a streaking experiment with isolated nanospheres (Süßmann and Kling 2011b). The plasmonic oscillations are excited with a few-cycle NIR laser pulse and probed with photoemission induced by an attosecond XUV pulse. The electron emission is detected along the polarization direction with a timeof-flight (TOF) spectrometer. In the simulation an Au sphere of $100 \mathrm{~nm}$ diameter excited with a laser pulse of 5 fs duration (FWHM of electric field) centered at $720 \mathrm{~nm}$ with peak intensity of $1 \times 10^{13}$ $\mathrm{W} \mathrm{cm}{ }^{-2}$ was considered (Süßmann and Kling 2011b).

The local field was calculated by finding the Mie solution at the central laser wavelength. The simulated near-field in figure 19(b) exhibits symmetry relative to the polarization vector of the incident field, with the maximum field enhancement at the poles along the polarization vector. The non-resonant excitation leads to a maximum field enhancement factor of 2.5 on the sphere surface. The electric field quickly decays from the surface with a typical length scale of tenth of nanometers. For the photoemission step, the XUV pulse duration and bandwidth were taken as 250 as, and $7 \mathrm{eV}$, respectively. The central photon energy was $105 \mathrm{eV}$, giving a $100 \mathrm{eV}$ initial electron energy. Electrons photoemitted by the XUV pulse were assumed to have initial velocity vectors parallel to the $y$-axis (along the TOF axis). The electron initial position is represented by the angle $\alpha$ in figure 19(b). The XUV pulse penetrates sufficiently deep into the nanoparticle for photoemission from the whole surface facing the TOF to be important. The relative emission from the back of the sphere was modeled using tabulated
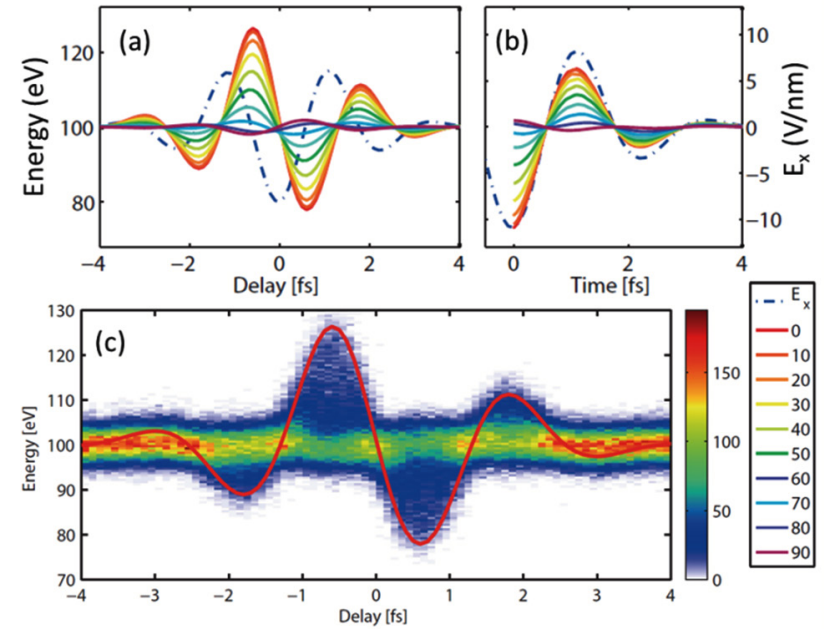

Figure 20. (a) Simulated streaking waveform for electrons emitted at different positions on a sphere of $100 \mathrm{~nm}$ diameter. (b) Effective field for electrons emitted at time $t_{e}=0$ at the same positions as in (a). (c) Simulated streaking spectrogram. The red line indicates the streaking curve for electrons emitted at the particle pole. Figure reused with permission from Süßmann and Kling (2011b). Copyright 2011 by American Physical Society.

material data to calculate the XUV transmission through the sphere. To achieve good statistics approximately $1.5 \times 10^{5}$ trajectories were initialized from the surface at each delay step.

Figure 20(a) shows streaking curves simulated for different electron initial positions. The plasmonic streaking field $E_{x}$ acting on the electrons emitted at $t_{e}=0$ is shown in figure 20(b), and the incident laser field is depicted by the blue dasheddotted line. The electrons emitted at the poles show a streaking curve shifted in phase by $\sim \pi / 2$ rad relative to the incident laser field, which is consistent with the ponderomotive picture of streaking. At larger values of $\alpha$, the streaking amplitude becomes smaller, but the phase of the streaking curve does not change significantly. For very large angles the phase shift relative to the laser field abruptly changes to approximately $\pi$ $\mathrm{rad}$. This emission position dependence of the streaking traces and the fields accelerating the photoemitted electrons results from the dipolar character of the near-field.

The resulting streaking trace is shown in figure 20(c). Contributions from trajectories originating from different parts of the surface result in a blurred spectrogram in comparison to typical streaking measurements in an atomic gas. Trajectory analysis shows that the electrons emitted from the poles contribute to the largest energy shifts of the photoelectron spectra (red line in figure 20(c)). The case considered here is in the ponderomotive streaking regime, resulting in a simple phase shift of the plasmonic field with respect to the streaking trace (see figure 18(a)). Once this phase shift has been determined from theory, full characterization of the plasmonic field can be performed experimentally. An analytical solution of equation (7) is generally not available, and in the intermediate streaking regime more complex streaking traces will be observed (Süßmann and Kling 2011b, Kelkensberg et al 2012). Here, numerical simulations combined with appropriate feedback may be employed for the retrieval of the spatiotemporal evolution of the near-fields. 

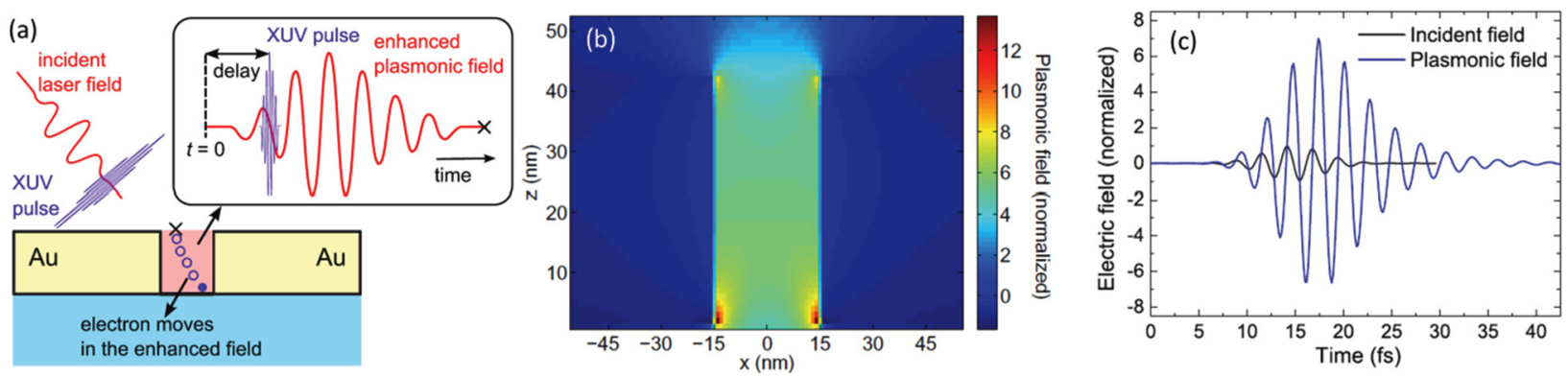

Figure 21. (a) Schematic representation of the experiment. (b) Calculated plasmonic field $E_{x}(x, z)$ in the gap between the antennas at the time of the maximum of the plasmonic field. (c) Time evolution of the incident laser field (black line) and the plasmonic field response $E_{x}(t)$ at the point $x=10 \mathrm{~nm}, z=0 \mathrm{~nm}$. Figure reused from Skopalová et al (2011) (CC BY 3.0).

\subsection{Attosecond streaking from nanoantennas}

We now turn our attention to streaking measurements on plasmonic nanostructures with more complex geometries. Plasmonic properties of surface based nanostructures have recently attracted attention due to their importance in applications ranging from chemical sensing (Anker et al 2008, Liu et al 2011) to the generation of XUV light (Kim et al 2008, Sivis et al 2013). The possibility of tracing plasmonic fields of an array of Au nanoantennas with attosecond streaking has been studied numerically (Skopalová et al 2011). Again, a few-cycle laser pulse excites the plasmonic field and a delayed attosecond pulse ionizes electrons that are then streaked in the plasmonic field (figure 21(a)). To calculate the time-dependent near-fields of the nanoantenna array three-dimensional finitedifference-time-domain (FDTD) simulations were performed for coupled antennas illuminated with a laser pulse of $5 \mathrm{fs}$ duration polarized in $x$-direction (Skopalová et al 2011). The dimensions and arrangement of the antenna elements were chosen such that the plasmon resonance of the nanostructure was centered at the carrier frequency of the incident laser pulse pulse $(800 \mathrm{~nm})$. As the spatial extension of the nanoplasmonic fields considered in the streaking simulation is much smaller than the wavelength of the $800 \mathrm{~nm}$ pulse, the nanoplasmonic field response can be described in the quasi-electrostatic field approximation. Thus, the same time dependence for all points in space was assumed and the plasmonic field is presented as a decomposition of its spatial and temporal components $E(x, z, t)=E(x, z) E(t)$. This assumption was supported by the FDTD simulations and is needed for reconstruction of the electric field from the streaking process. The spatial distribution of the plasmonic field $E_{x}(x, z)$ exhibits maxima of the field enhancement near the corners of the gap (figure 21(b)). The time dependent evolution of the plasmonic field shows a resonance response with field oscillations lasting for more than 10 fs after the excitation pulse (figure 21(c)).

The initial energy distribution of photoelectrons was simulated by convolution of 580 as Gaussian pulse at $90 \mathrm{eV}$ photon energy with a spectrum obtained from narrow-line $\mathrm{x}$-ray photoelectron spectroscopy measurements in $\mathrm{Au}$ and corrected for the energy-dependence of the ionization cross-section. Equation (7) was numerically solved for electrons emitted at different positions along the $x=15 \mathrm{~nm}, z=0-40 \mathrm{~nm}$ surface, and the streaking spectrogram in figure 22(a) was obtained by averaging over these different initial positions.
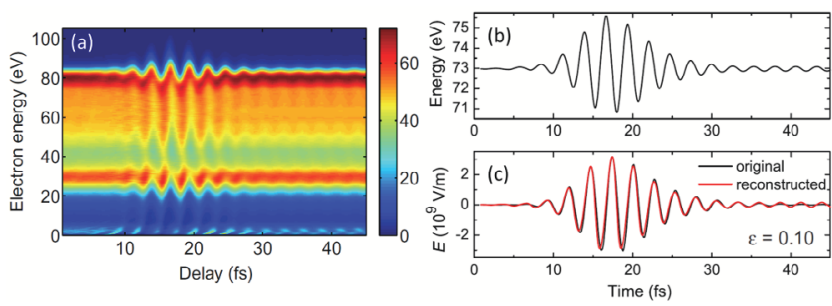

Figure 22. (a) Simulated streaking spectrogram of Au nanoantenas. (b) Center of mass of the final electron energy as a function of the time delay. The spectra were integrated over the energy range $60-110 \mathrm{eV}$ and the initial electron position was averaged over the $z$-direction. (c) Original field (black) and reconstructed field (red). The original field was normalized to have the same maximum as the reconstructed field. Figure reused from Skopalová et al (2011) (CC BY 3.0).

The spectrogram displays streaking of photoelectrons emitted from both the valence and $5 \mathrm{p}$ bands of $\mathrm{Au}$, and resembles conventional streaking in gas indicating that the majority of electrons are streaked in the ponderomotive regime. To retrieve the plasmonic field evolution, the center-of-mass of the valence band was found as a function of time delay. The energy shift of the valence band (figure 22(b)) approximately follows the vector potential of the plasmonic field, allowing the electric field to be obtained by differentiating the center-ofmass curve as a function of the time delay. The reconstructed field is in close agreement with the original plasmonic field (figure 22(c)). It should be noted that the amplitude of the reconstructed waveform can be underestimated because of the finite XUV pulse duration. Similarly, for isolated rectangular nanoparticles it was found from simulations in Borisov et al (2012) that the oscillations in the streaking spectrogram closely followed the plasmonic field.

Disentangling electrons from different emission positions indicates that not all of the electrons are streaked in the ponderomotive regime (Skopalová et al 2011). In particular, high energy electrons emitted far from the substrate (i.e. high initial $z$ position) and at short time-delays can escape fast enough to enter the intermediate streaking regime. This distorts the delay-energy relationship for these initial positions. To further understand the complex spatio-temporal structure of plasmonic near-fields, photoelectron emission microscopy (PEEM) setups aiming to combine nanometric spatial resolution with attosecond time resolution are currently being developed (Stockman et al 2007, Mikkelsen et al 2009, Chew 
et al 2012, Gong et al 2015). Plasmon propagation could be investigated with attosecond temporal resolution and micron spatial resolution by spatially displacing the NIR and XUV pulses in streaking experiments (Lupetti et al 2014).

\subsection{Attosecond streaking at nanotapers}

While the numerous theoretical studies described above indicated that nano-localized fields can be characterized with attosecond precision using streaking, the experimental implementation proved challenging. The linear XUV-induced photoemission process typically probes a much larger area than the nanoscale region of interest, and the streaking trace can be distorted because electrons emitted from different regions are streaked by different local fields. The absolute number of electrons emitted is also very small due to small sample sizes. However, a recent advance has been made in this area with the first streaking measurements performed on a nanostructure (Förg et al 2016). By combining the measurements with a thorough analysis of the near-field spatial distribution and photoelectron trajectories, the authors were able to characterise the near-fields surrounding a gold nanotaper with attosecond precision.

In the experiments, co-propagating $4.5 \mathrm{fs}$ NIR laser pulses at $720 \mathrm{~nm}$ central wavelength, and isolated 220 as XUV pulses at $95 \mathrm{eV}$ central energy, were generated and used to perform streaking measurements on a gold nanotaper. A scanning electron microscopy (SEM) image of the sample is shown in figure 23(a). The XUV focal spot (5 $\mu$ m diameter) was centred on the tip apex ( $100 \mathrm{~nm}$ radius of curvature), although no significant XUV induced photoelectron signal was detected from the apex itself due to its small surface area. The XUV effectively probed the near-fields surrounding the nanowire taper within a distance of $2.5 \mu \mathrm{m}$ from the apex and with a diameter tapering from $200 \mathrm{~nm}$ to $640 \mathrm{~nm}$. The NIR polarization was aligned with the nanotaper axis.

Theoretical considerations indicated that the photoelectrons were streaked in the ponderomotive regime. The nearfields, calculated using an FDTD method, are shown in figure 23(b). The near-fields in the probed region have a high degree of spatial homogeneity in amplitude and phase, and are shifted in phase by $0.8 \mathrm{rad}$ (corresponding to a temporal shift of 300 as) with respect to the incident NIR pulse.

The experimental streaking trace from the nanotaper is shown in figure 23(c). The gas-phase streaking measurement in figure 23(d) gives the phase of the incident NIR pulse as a reference. The nanotaper streaking trace is shifted with respect to the gas phase streaking measurement by $\Delta t=(250 \pm 50)$ as. The measured shift was free from any significant contribution from photoemission time delays because the NIR field polarisation was parallel to the sample surface, resulting in a continuous electric field across the surface. The measured shift is in agreement with the theoretical value from MonteCarlo simulations of photoelectron trajectories, confirming that the measurements successfully probed the near-fields around the nanotaper. The electric near-field retrieved from

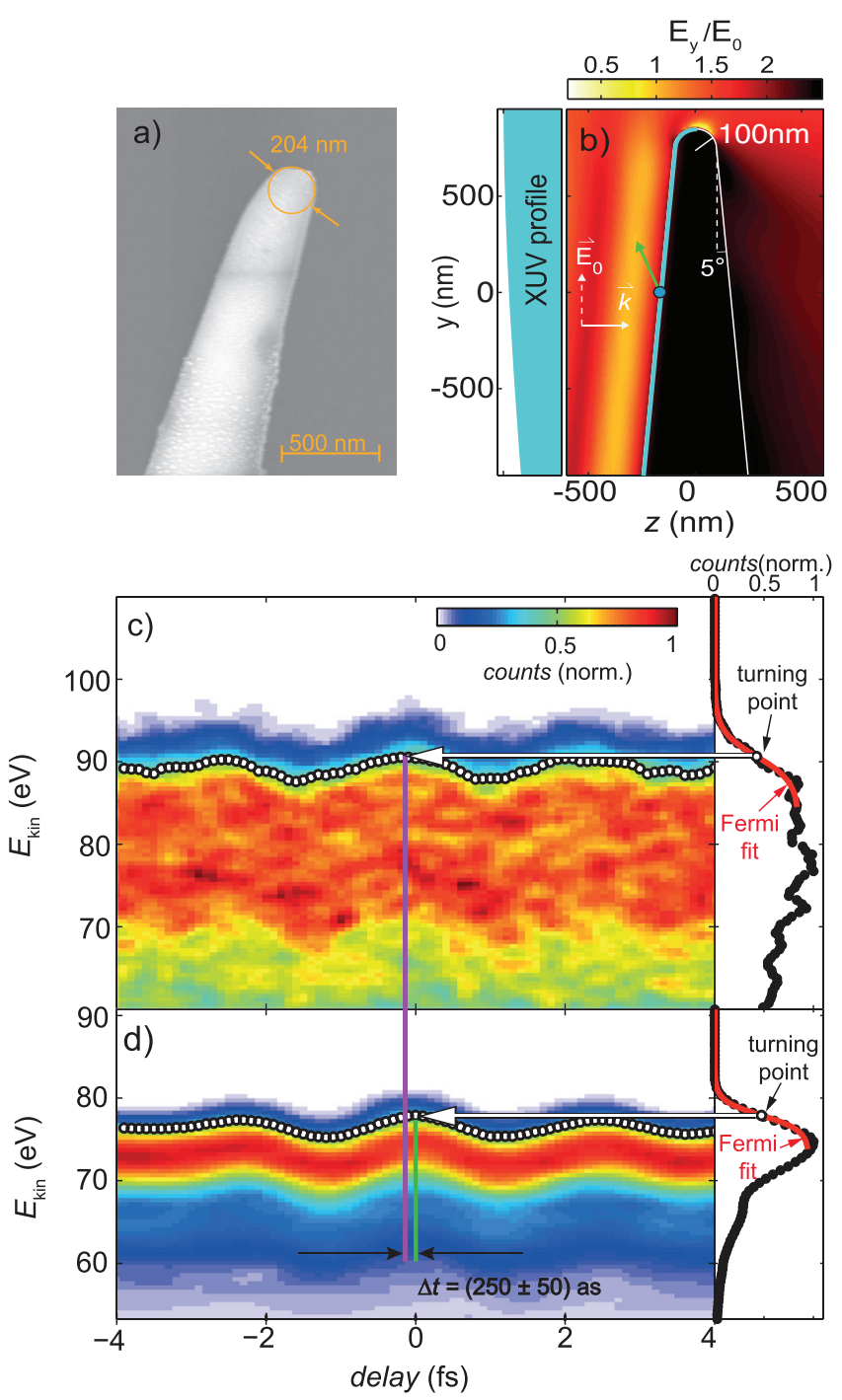

Figure 23. (a) SEM image of nanotaper sample. (b) Normalized field strength of the field component parallel to the nanotaper axis, calculated using an FDTD method. The blue line shows the region of the sample illuminated by the XUV, and the spatial profile of the XUV focus is shown on the left. (c) Streaking measurement from the nanotaper sample. The energy shift of the streaking trace versus the time delay between the XUV and NIR pulses, shown by the white data points, was extracted by fitting a Fermi function (red) to the cut-off. (d) Reference streaking measurement in neon gas. The neon streaking trace is shifted in time by $\Delta t=(250 \pm 50)$ as relative to the nanotaper trace. Figure reused from Förg et al (2016) with by permission from Macmillan Publishers Ltd: Nature Communications, Copyright (2016).

streaking measurements is shown in figure 24 , and is in close agreement with the field expected from calculations (also shown in the same figure).

The experiments open the door to using the same approach to measure local near-fields and attosecond plasmon dynamics in more complex nanostructures, such as ultrafast optoelectronic components. Future characterisation measurements of the electric field around the tip apex should furthermore yield a richer understanding of the physics discussed in section 4, involving electron acceleration at nanoscale needle tips. 


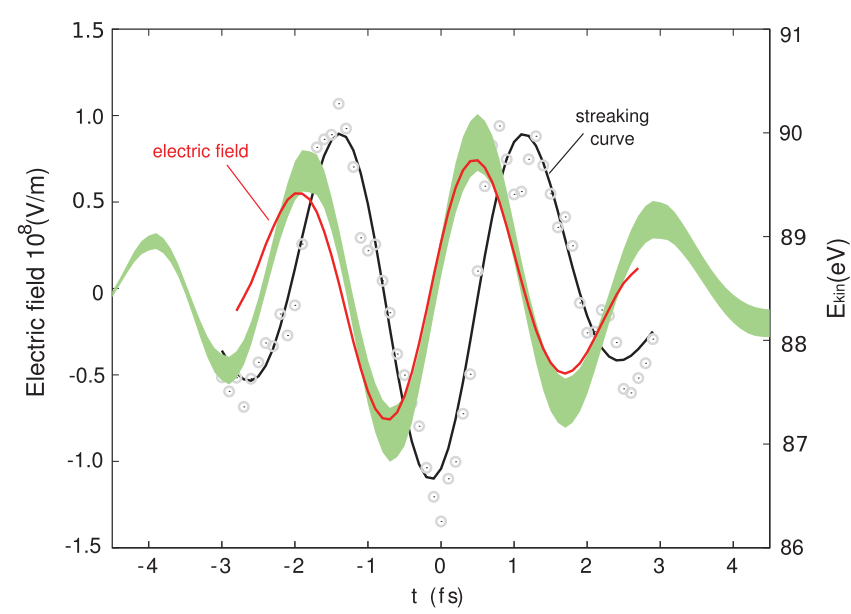

Figure 24. Electric near-field (red) around the nanotaper, extracted from streaking measurements. The energy shift of the streaking measurement (data points), the Fourier filtered shift (streaking curve, black), and the calculated near-field (green shaded area) are also shown. Figure reused from Förg et al (2016) by permission from Macmillan Publishers Ltd: Nature Communications, Copyright (2016)

\section{Extreme-ultraviolet light generation in plasmonic nanofields}

The extreme local field enhancements that can be achieved by concentrating light into nanoscale volumes using plasmonic nanostructures have attracted significant interest from the ultrafast physics community. One of the applications that has generated the most excitement is the possibility to generate XUV light at high $(\mathrm{MHz})$ repetition rates without need for an enhancement cavity. This work was initiated by Kim et al (2008), where pulses from a femtosecond oscillator (75 $\mathrm{MHz}$ repetition rate) were focused onto an array of bow-tie Au nanoantennas on a sapphire substrate and surrounded by argon gas. The bow-tie structures acted as resonant antennas concentrating the optical energy in the gaps between adjacent elements (see figure 25). The estimated intensity enhancement of more than $20 \mathrm{~dB}$ was sufficient enough to produce XUV radiation in the argon with wavelengths down to $47 \mathrm{~nm}$.

The work of Kim et al (2008) triggered a number of further experimental (Park et al 2011, 2013, Kim et al 2012, Pfullmann et al 2013, Sivis et al 2012, 2013) and theoretical (Stebbings et al 2011, Husakou et al 2011a, Yavuz et al 2012, Ciappina et al 2012a, 2012b, 2013c, 2014c, Shaaran et al 2012a, 2012b, 2013a, Pérez-Hernández et al 2013) efforts in a similar direction. Despite initial success in the observation of XUV light from bow-tie nanostructures the origin of the observed radiation remained debated. The study of Sivis et al (2013) significantly deepened the understanding of the mechanisms responsible for the observed plasmon enhanced light emission. The authors compared the emission characteristics using nanostructures illuminated with low energy laser pulses from an oscillator to those obtained in conventional gas target with amplified high energy pulses.

Spectra measured from different bow-tie nanoantenna samples are presented in figure 26(a). The authors identify the most pronounced features as atomic line emission (ALE) from neutral and singly ionized Ar atoms. The incoherent nature of

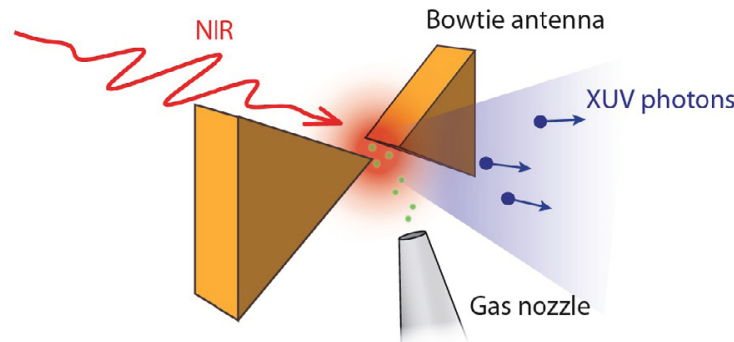

Figure 25. Schematic representation of a bow-tie nanostructure for XUV generation. The structure is illuminated with a few cycle NIR laser field and gas is injected into the antenna gap. Figure reused with permission from Süßmann et al (2014). Copyright 2014 by John Wiley \& Sons, Inc.

these spectra is confirmed by the close agreement with ALE spectra measured in Ar gas illuminated with amplified pulses (figure 26(d)), detected in the direction perpendicular to the laser propagation. In contrast, the emission from the gas target in the laser propagation direction clearly shows high-order harmonic radiation (figure 26(e)).

Intensity dependent measurements on the nanostructures indicate local field intensities up to and beyond the damage threshold of the material. These intensities would in principle be sufficient for coherent HHG in Ar gas (figure 26(c)). The lack of high-harmonic emission in the measured spectra thus indicates that although the local intensities are clearly above the threshold for HHG, the small nanostructure generation volumes are insufficient for the coherent build-up of any noticeable HHG signal. A rough estimate using the actual experimental conditions indicates that the expected HHG signal from the nanostructure target is about $6 \times 10^{-3}$ smaller than the ALE.

Later work employed three dimensional tapered waveguides for XUV generation by adiabatically nanofocused SPPs (Park et al 2011), where NIR pulses from a femtosecond oscillator were focused on the inlet of the waveguide with an intensity of $\sim 10^{11} \mathrm{~W} \mathrm{~cm}^{-2}$. These pulses excite an SPP wave that propagates inside the waveguide towards the exit. The parameters of the waveguide were optimized using FDTD simulations and a peak intensity enhancement factor of more than $20 \mathrm{~dB}$ relative to the incident field was obtained in a near cylindrical volume of diameter $240 \mathrm{~nm}$ and length $450 \mathrm{~nm}$ near the exit aperture. This volume is about three orders of magnitude larger than the generation volume (the volume containing an intensity enhancement of $>20 \mathrm{~dB}$ ) of a single bow-tie element used in previous work of Kim et al (2008).

By back-filling the waveguide with Xe gas, XUV generation up to $70 \mathrm{eV}$ photon energy was achieved (Park et al 2011). Compared to previous studies using bow-tie nanoantennas the three dimensional waveguide displays more than an order of magnitude higher XUV generation efficiency. In addition the waveguide fabricated on a cantilever microstructure is much less susceptible to thermal and optical damage. The origin of the observed radiation is, however, again disputed. Experimental investigations in Sivis and Ropers (2013) indicate that while a sufficient intensity for HHG is achieved at the focus of the waveguide, the length of the guide 


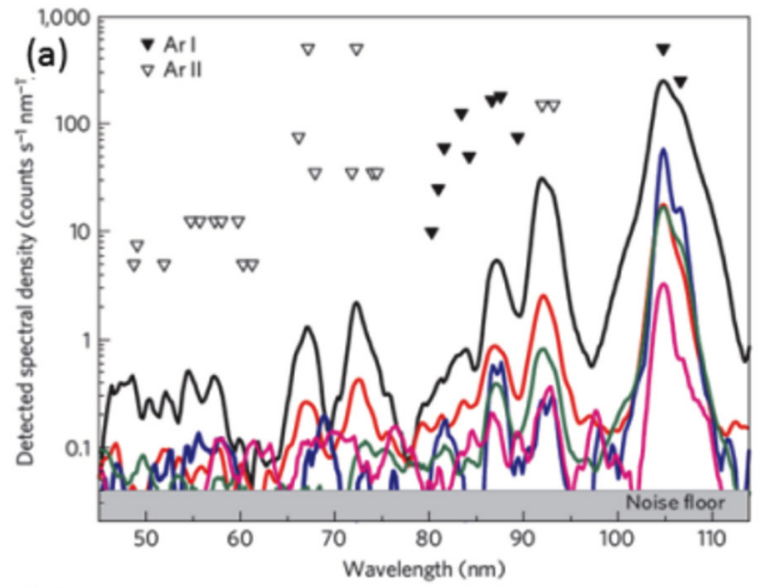

(b)

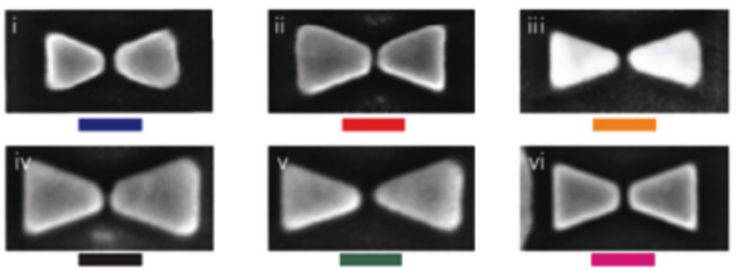

(c)
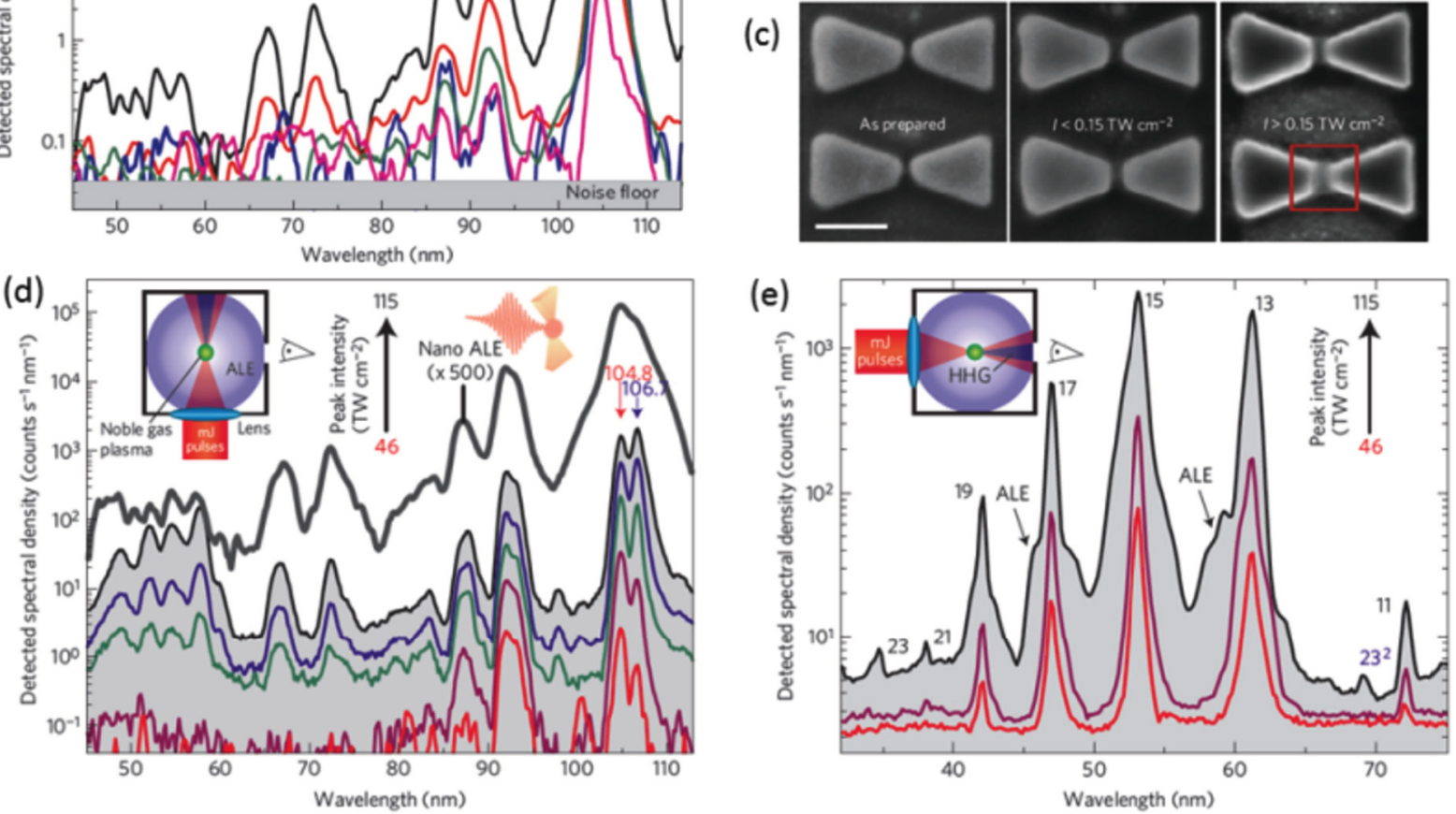

Figure 26. (a) XUV spectra measured in bow-tie nanostructures exposed to Ar gas. Triangles indicate expected ALE transitions for neutral (filled) and singly ionized (open) Ar atoms. (b) SEM images of the bow-tie antennas used in the measurements presented in (a). (c) SEM images of nanoantennas $i v$ after the preparation, after exposure for several hours to laser intensity up to $0.15 \mathrm{TW} \mathrm{cm}^{-2}$, and after exposure for a few minutes to laser intensity up to $0.3 \mathrm{TW} \mathrm{cm} \mathrm{m}^{-2}$ (from left to right respectively). (d) Intensity dependent spectra measured in Ar gas in the direction perpendicular to the laser beam propagation. For comparison a spectrum measured in bow-tie nanostructures is presented as a thick grey line. (e) Intensity dependent spectra measured along the laser beam propagation direction. Figure reused with permission from Sivis et al (2013). Copyright 2013 Macmillan Publishers Ltd: Nature Physics.

is insufficient for a significant buildup of the signal. HHG at $\mathrm{MHz}$ repetition rates in enhanced plasmonic fields remains an attractive prospect. However, efficient generation will require substantially higher gas pressures and larger interaction volumes. Meanwhile, the incoherent enhanced ALE that has been successfully generated in plasmonic near-fields could nevertheless find applications in areas such as near-field imaging.

\section{Theoretical approaches}

In the next sections we describe the theoretical approaches we have developed to tackle strong field processes driven by spatially inhomogeneous laser fields. We put particular emphasis on the HHG and ATI, but we include at the end an incipient attempt to treat multielectronic phenomena.

\subsection{HHG driven by spatially inhomogeneous fields}

HHG driven by plasmonic-enhanced laser fields, generated starting from engineered nanostructures or nanoparticles, requires no extra amplification stages due to the fact that, by exploiting surface plasmon resonances, the input driving electric field can be enhanced by more than $20 \mathrm{~dB}$ (corresponding to an increase in the intensity of several orders of magnitude). As a consequence of this enhancement, the threshold laser intensity for HHG generation in noble gases is largely exceeded and the pulse repetition rate remains unaltered. In addition, the high-harmonics radiation generated from each nanosystem acts as a pointlike source, enabling a high collimation or focusing of this coherent radiation by means of (constructive) interference. This fact opens a wide range of possibilities to spatially arrange nanostructures to enhance or shape the spectral and spatial properties of the harmonic radiation in numerous ways (Kim et al 2008, Park et al 2011, Pfullmann et al 2013).

Due to the nanometric size of the so-called plasmonic 'hot spots', i.e. the spatial region where the electric field reaches its highest intensity, one of the main theoretical assumptions, namely the spatial homogeneity of the driven electric field, should be removed (see section 1.2). Consequently, both the analytical and numerical approaches to study laser-matter processes in atoms and molecules, in particular $\mathrm{HHG}$, need to be modified to treat adequately this different scenario and allow now for a spatial dependence in the laser electric 
field. Several authors have addressed this problem recently Cao et al (2014), Chacón et al (2015), Ciappina et al (2012a, 2012b), (2014a, 2015), (2012, 2014b), (2014c, 2013a), (2013b, 2013c), Ebadi (2014), Feng and Liu (2015), Feng et al (2013), Fetić et al (2012), He et al (2013), Husakou and Herrmann (2014), Husakou et al (2011a, 2011b), Luo et al (2013a, 2013b), (2013c), Pérez-Hernández et al (2013), Shaaran et al (2013a, 2012a), (2012b, 2013b), Wang et al (2014, 2013), Yavuz (2013), Yavuz et al (2012, 2015), Yu et al (2015), Zang et al (2013). As we will show below, this new characteristic affects considerably the electron dynamics and this is reflected on the observables, in the case of this section the HHG spectra.

\subsection{Quantum approaches}

The dynamics of a single active atomic electron in a strong laser field takes place along the polarization direction of the field, when linearly polarized laser pulses are employed. It is then justifiable to model the HHG in a 1D spatial dimension by solving the time dependent Schrödinger equation (1D-TDSE) (Ciappina et al 2012b):

$$
\begin{aligned}
\mathrm{i} \frac{\partial \Psi(x, t)}{\partial t} & =\mathcal{H}(t) \Psi(x, t) \\
& =\left[-\frac{1}{2} \frac{\partial^{2}}{\partial x^{2}}+V_{\mathrm{a}}(x)+V_{\mathrm{l}}(x, t)\right] \Psi(x, t),
\end{aligned}
$$

where in order to model an atom in $1 \mathrm{D}$, it is common to use soft core potentials, which are of the form:

$$
V_{\mathrm{a}}(x)=-\frac{1}{\sqrt{x^{2}+b^{2}}},
$$

where the parameter $b$ allows us to modify the ionization potential $I_{\mathrm{p}}$ of the ground state, fixing it as close as possible to the value of the atom under consideration. We consider the field to be linearly polarized along the $x$-axis and modify the interaction term $V_{1}(x, t)$ in order to treat spatially nonhomogeneous fields, while maintaining the dipole character. Consequently we write

$$
V_{1}(x, t)=-E(x, t) x
$$

where $E(x, t)$ is the laser electric field defined as

$$
E(x, t)=E_{0} f(t)(1+\varepsilon h(x)) \sin \left(\omega t+\phi_{\mathrm{CEP}}\right) .
$$

In equation (11), $E_{0}, \omega$ and $\phi_{\mathrm{CEP}}$ are the laser electric field peak amplitude, the frequency of the laser pulse and the CEP, respectively. We refer to $\sin (\cos )$-like laser pulses where $\phi_{\mathrm{CEP}}=0\left(\phi_{\mathrm{CEP}}=\pi / 2\right)$. The pulse envelope is given by $f(t)$ and $\varepsilon$ is a small parameter that characterizes the inhomogeneity strength. The function $h(x)$ represents the functional form of the spatial nonhomogeneous field and, in principle, could take any form and be supported by the numerical algorithm (for details see e.g. Ciappina et al (2012a, 2012b)). Most of the approaches use the simplest form for $h(x)$, i.e. the linear term: $h(x)=x$. This choice is motivated by previous investigations (Husakou et al 2011a, Ciappina et al 2012b, Yavuz et al
2012), but nothing prevents to use more general functional forms for $h(x)^{17}$.

The 1D-TDSE can be solved numerically by using the Crank-Nicolson scheme in order to obtain the time propagated electronic wavefunction $\Psi(x, t)$. Once $\Psi(x, t)$ is found, we can compute the HHG spectrum by Fourier transforming the dipole acceleration of the active electron. One of the main advantages of the 1D-TDSE is that we are able to include any functional form for the spatial variation of the plasmonic field. For instance, we have implemented linear (Ciappina et al 2012b) and real (parabolic) plasmonic fields (Ciappina et al 2012a), as well as near-fields with exponential decay (evanescent fields) (Shaaran et al 2013a).

An extension of the above described approach is to solve the three dimensional TDSE (3D-TDSE) and to include in the laser-electron potential the spatial variation of the laser electric field. For only one active electron we need to deal with 3 spatial dimensions and, due to the cylindrical symmetry of the problem, we are able to separate the electronic wavefunction in spherical harmonics, $Y_{l}^{m}$ and consider only terms with $m=0$ (see below).

The 3D-TDSE in the length gauge can be written:

$$
\begin{aligned}
\mathrm{i} \frac{\partial \Psi(\mathbf{r}, t)}{\partial t} & =H \Psi(\mathbf{r}, t) \\
& =\left[-\frac{\nabla^{2}}{2}+V_{\mathrm{SAE}}(\mathbf{r})+V_{l}(\mathbf{r}, t)\right] \Psi(\mathbf{r}, t),
\end{aligned}
$$

where $V_{\mathrm{SAE}}(\mathbf{r})$ is the atomic potential in the single active electron (SAE) approximation and $V_{l}(\mathbf{r}, t)$ the laser-electron coupling (see below). The time-dependent electronic wave function $\Psi(\mathbf{r}, t)$, can be expanded in terms of spherical harmonics:

$$
\begin{aligned}
\Psi(\mathbf{r}, t) & =\Psi(r, \theta, \phi, t) \\
& \approx \sum_{l=0}^{L-1} \sum_{m=-l}^{l} \frac{\Phi_{l m}(r, t)}{r} Y_{l}^{m}(\theta, \phi)
\end{aligned}
$$

where the number of partial waves depends on each specific case. Here, in order to assure the numerical convergence, we have used up to $L \approx 250$ in the most extreme case $\left(I \sim 5 \times 10^{14}\right.$ $\mathrm{W} \mathrm{cm}^{-2}$ ). In addition, due to the fact that the plasmonic field is linearly polarized, the magnetic quantum number is conserved and consequently in the following we can consider only $m=0$ in equation (13). This property considerably reduces the complexity of the problem. In here, we consider $z$ as a polarization axis and we take into account that the spatial variation of the electric field is linear with respect to the position. As a result, the coupling $V_{l}(\mathbf{r}, t)$ between the atomic electron and the electromagnetic radiation reads

\footnotetext{
${ }^{17}$ The actual spatial dependence of the enhanced near-field in the surrounding of a metal nanostructure can be obtained by solving the Maxwell equations incorporating both the geometry and material properties of the nanosystem under study and the input laser pulse characteristics (see e.g. Ciappina et al (2012a)). The electric field retrieved numerically is then approximated using a power series $h(x)=\sum_{i=1}^{N} b_{i} x^{i}$, where the coefficients $b_{i}$ are obtained by fitting the real electric field that results from a finite element simulation. Furthermore, in the region relevant for the strong field physics and electron dynamics and in the range of the parameters we are considering, the electric field can be indeed approximated by its linear dependence.
} 


$$
V_{l}(\mathbf{r}, t)=\int^{\mathbf{r}} d \mathbf{r}^{\prime} \cdot \mathbf{E}\left(\mathbf{r}^{\prime}, t\right)=E_{0} z(1+\varepsilon z) f(t) \sin \left(\omega t+\phi_{\mathrm{CEP}}\right)
$$

As in previous investigations, the parameter $\varepsilon$ defines the 'strength' of the inhomogeneity and has units of inverse length (see also Ciappina et al (2012b), Husakou et al (2011a), Yavuz et al (2012)). For modeling short laser pulses in equation (14), we use a sin-squared envelope $f(t)$ of the form $f(t)=\sin ^{2}\left(\frac{\omega t}{2 n_{p}}\right)$, where $n_{p}$ is the total number of optical cycles. As a result, the total duration of the laser pulse will be $T_{p}=n_{p} \tau_{L}$ where $\tau_{L}=2 \pi / \omega$ is the laser period. We focus our analysis on a hydrogen atom, i.e. $V_{\mathrm{SAE}}(\mathbf{r})=-1 / r$ in equation (12), and we also assume that before switching on the laser $(t=-\infty)$ the target atom is in its ground state $(1 s)$, whose analytic form can be found in a standard textbook. Within the SAE approximation, however, our numerical scheme is tunable to treat any complex atom by choosing the adequate effective (Hartree-Fock) potential $V_{\mathrm{SAE}}(\mathbf{r})$, and finding the ground state by the means of numerical diagonalization or imaginary time propagation.

Next, we will show how the spatial inhomogeneity modifies the equations which model the laser-electron coupling. Inserting equation (13) into equation (12) and considering that,

$$
\cos \theta Y_{l}^{0}=c_{l-1} Y_{l-1}^{0}+c_{l} Y_{l+1}^{0}
$$

and

$\cos ^{2} \theta Y_{l}^{0}=c_{l-2} c_{l-1} Y_{l-1}^{0}+\left(c_{l-1}^{2}+c_{l}^{2}\right) Y_{l}^{0}+c_{l} c_{l+1} Y_{l+2}^{0}$,

where

$$
c_{l}=\sqrt{\frac{(l+1)^{2}}{(2 l+1)(2 l+3)}},
$$

we obtain a set of coupled differential equations for each of the radial functions $\Phi_{l}(r, t)$ :

$$
\begin{aligned}
\mathrm{i} \frac{\partial \Phi_{l}}{\partial t}= & {\left[-\frac{1}{2} \frac{\partial^{2}}{\partial r^{2}}+\frac{l(l+1)}{2 r^{2}}-\frac{1}{2}\right] \Phi_{l} } \\
& +\varepsilon r^{2} E(t)\left(c_{l}^{2}+c_{l-1}^{2} \Phi_{l}\right. \\
& +r E(t)\left(c_{l-1} \Phi_{l-1}+c_{l} \Phi_{l+1}\right) \\
& +\varepsilon r^{2} E(t)\left(c_{l-2} c_{l-1} \Phi_{l-2}+c_{l} c_{l+1} \Phi_{l+2}\right) .
\end{aligned}
$$

Equation (18) is solved using the Crank-Nicolson algorithm considering the additional term, i.e. equation (16) due to the spatial inhomogeneity. As can be observed, the degree of complexity will increase substantially when a more complex functional form for the spatial inhomogeneous laser electric field is used. For instance, the incorporation of only a linear term couples the angular momenta $l, l \pm 1, l \pm 2$, instead of $l, l \pm 1$, as in the case of conventional (spatial homogeneous) laser fields.

We have also made studies on helium because a majority of experiments in HHG are carried out in noble gases. Nonetheless, other atoms could be easily implemented by choosing the appropriate atomic model potential $V_{\mathrm{SAE}}(\mathbf{r})$. After time propagation of the electronic wavefunction, the HHG spectra can be computed in an analogous way as in the case of the 1D-TDSE. Due to the complexity of the problem, only simulations with nonhomogeneous fields with linear spatial variations along the laser polarization in the 3D-TDSE have been studied. This, however, is enough to confirm that even a small spatial inhomogeneity significantly modifies the HHG spectra (for details see Pérez-Hernández et al (2013)).

\subsection{Semiclassical approach}

An independent approach to compute HHG spectra for atoms in intense laser pulses is the strong field approximation (SFA) or Lewenstein model (Lewenstein et al 1994). The main ingredient of this approach is the evaluation of the time-dependent dipole moment $\mathbf{d}(t)$. Within the single active electron (SAE) approximation, it can be calculated starting from the ionization and recombination transition matrices combined with the classical action of the laser-ionized electron moving in the laser field. The SFA approximation has a direct interpretation in terms of the so-called three-step or simple man's model (Corkum 1993, Lewenstein et al 1994) (see section 1.1).

Implicitly the Lewenstein model deals with spatially homogeneous electric and vector potential fields, i.e. fields that do not experience variations in the region where the electron dynamics takes place. In order to consider spatial nonhomogeneous fields, the SFA approach needs to be modified accordingly, i.e. the ionization and recombination transition matrices, joint with the classical action, now should take into account this new feature of the laser electric and vector potential fields (for details see Ciappina et al (2012b), Shaaran et al (2013b)).

\subsection{Classical framework}

Important information such as the HHG cutoff and the properties of the electron trajectories moving in the oscillatory laser electric field, can be obtained solving the classical Newton-Lorentz equation for an electron moving in a linearly polarized electric field. Specifically, we find the numerical solution of

$$
\ddot{x}(t)=-\nabla_{x} V_{1}(x, t),
$$

where $V_{1}(x, t)$ is defined in equation (10) with the laser electric field linearly polarized in the $x$ axis. For fixed values of ionization times $t_{i}$, it is possible to obtain the classical trajectories and to numerically calculate the times $t_{r}$ for which the electron recollides with the parent ion. In addition, once the ionization time $t_{i}$ is fixed, the full electron trajectory is completely determined (for more details about the classical model see Ciappina et al (2014a)).

The following conditions are commonly set (the resulting model is also known as the simple man's model): (i) the electron starts with zero velocity at the origin at time $t=t_{i}$, i.e. $x\left(t_{i}\right)=0$ and $\dot{x}\left(t_{i}\right)=0$; (ii) when the laser electric field reverses its direction, the electron returns to its initial position, i.e. recombines with the parent ion, at a later time, $t=t_{r}$, i.e. $x\left(t_{r}\right)=0 . t_{i}$ and $t_{r}$ are known as ionization and recombination times, respectively. The electron kinetic energy at $t_{r}$ can be obtained from the usual formula $E_{k}\left(t_{r}\right)=\dot{x}\left(t_{r}\right)^{2} / 2$, and, finding 
the value of $t_{r}$ (as a function of $t_{i}$ ) that maximizes this energy, we find that the HHG cutoff is given by $n_{c} \omega_{0}=3.17 U_{p}+I_{\mathrm{p}}$, where $n_{c}$ is the harmonic order at the cutoff, $\omega_{0}$ is the laser frequency, $U_{p}$ is the ponderomotive energy and $I_{\mathrm{p}}$ is the ionization potential of the atom or molecule under consideration. It is worth mentioning that the HHG cutoff will be extended when spatially inhomogeneous fields are employed.

\subsection{Classical trajectory Monte Carlo (CTMC)}

In order to achieve quantitative accuracy for realistic systems, the classical framework may be coupled to accurate near-field, ionization, and scattering models into the classical trajectory Monte-Carlo (CTMC) scheme. Such scheme has several advantages in comparison with quantum simulations. First of all, even though the solution of the 3D-TDSE is possible in the SAE approximation under simplifying assumptions (see section 7.2 above), a detailed description of complex geometries, coupled with realistic near-fields, is still out of reach for purely quantum methods. In addition, the (bulk) scattering and multi-electron effects complicate the quantum treatment to the extent that they are neglected in virtually every quantum calculation.

Similarly to the simple man's model of HHG, a CTMC simulation starts with the ionization of electrons which is typically described stochastically using methods based on a simplified quantum treatment, most often the Fowler-Nordheim theory (Fowler and Nordheim 1928) which is closely related to the ADK theory of ionization of atoms (Ammosov et al 1986). Multiphoton effects may be accounted for within the framework of Fowler-Nordheim theory by considering the response of the electron distribution function of the nano-device to the laser field (Yanagisawa et al 2009, 2011, Yanagisawa et al 2016) or with more refined theoretical frameworks (Yalunin et al 2011).

After the ionization, the electrons are propagated using classical equations of motion in the near-field. For complex geometries, the near-field may be obtained in the time domain, e.g. with FDTD methods or semi-analytically in the frequency domain, e.g. with the multiple multipole programs (MMP) (Hafner 1999). For nanospheres, simpler analytic Mie theory may be employed. For details see section 2.1.

For low intensities or when only qualitative results are sought, the electron-electron repulsion (space charge) may be neglected (Krüger et al 2011, Herink et al 2012, Wachter et al 2012, Dombi et al 2013). For higher intensities and accuracy, the space charge effects may be treated explicitly (Piglosiewicz et al 2014, Yanagisawa et al 2016) or using the mean-field approximation (Süßmann et al 2015). While the mean-field approximation scales linearly with the number of electrons ionized $N_{\mathrm{el}}$, the explicit treatment leads to $N_{\mathrm{el}}^{2}$ dependence. This unfavorable scaling may be alleviated without significantly compromising the accuracy by using methods like fast multipole method (FMM) or Barnes-Hut tree based methods with $N \log N$ scaling (Winkel et al 2012, Arnold et al 2013, Bolten et al 2016). In addition, the response of the nano-device on the ionized electron cloud should be taken into account. Simple analytic formulas may be used for planar and spherical geometries (Yanagisawa et al 2016). For complex geometries, the electrostatic problem may be solved numerically (Zherebtsov et al 2011).

The description of the re-collision of an electron with a nano-device ranges from a simple surface reflection (using several approximations) (Krüger et al 2011, Wachter et al 2012, Dombi et al 2013, Park et al 2013) through approaches relying on a variety of empirical and fitted parameters (Yanagisawa et al 2016) to a propagation of electrons inside the nano-device using the Langevin dynamics with stochastic events representing elastic and inelastic scattering (Lemell et al 2009, Süßmann et al 2015). The probability $P$ of a particular scattering event in the time interval $(t, t+\mathrm{d} t)$ is given by $P=v \cdot \mathrm{d} t / \lambda_{m}$, where $v$ is the electron velocity and $\lambda_{m}$ is the mean-free path. For elastic scattering, mean free paths and scattering differential cross sections (DCSs) may be obtained from quantum or time-dependent density functional theory (TDDFT) calculations for an electron interacting with an isolated atom and combined with, e.g. the muffin-tin approximation to model the solid state material (Salvat et al 2005). The inelastic scattering can be described as an interaction of the electron with a dielectric medium defined by the complex-wave vector $q$ and frequency $\omega$-dependent-dielectric function $\epsilon(q, \omega)$ which may be computed using the electron gas model (Lindhard 1954, Mermin 1970, Lafrate et al 1980), extended from experimentally measured optical energy loss function via Drude models (Tókési et al 2001, Solleder et al 2007, Da et al 2014) or calculated ab initio (typically employing TDDFT). Alternatively, empirical formulas may be used to describe some aspects of inelastic scattering (Lotz 1967, Fernandez-Varea et al 1993).

\section{Selected results}

In the following sections we present a brief summary of the results reported in several recent published works. In these articles, different noble gases ( $\mathrm{He}, \mathrm{Ar}$ and $\mathrm{Xe}$ ) are used as atomic targets located in the vicinity of metal nanotips and nanoparticles and the HHG generated by them were studied and characterized. In addition, we include here predictions for the generation of coherent harmonic radiation directly from the metal surface of a nanotip.

\subsection{Spatially (linear) nonhomogeneous fields and electron confinement}

In this section we summarize the study carried out in Ciappina et al (2012b) where it is shown that both the inhomogeneity of the local fields and the constraints in the electron movement, play an important role in the HHG process and lead to the generation of even harmonics and a significant increase in the HHG cutoff, more pronounced for longer wavelengths. In order to understand and characterize these new HHG features we employ two of the different approaches mentioned above: the numerical solution of the 1D-TDSE (see panels (a)-(d) in figure 27) and the semiclassical approach known as strong field approximation (SFA). Both approaches predict comparable results and describe satisfactorily the new features, but 

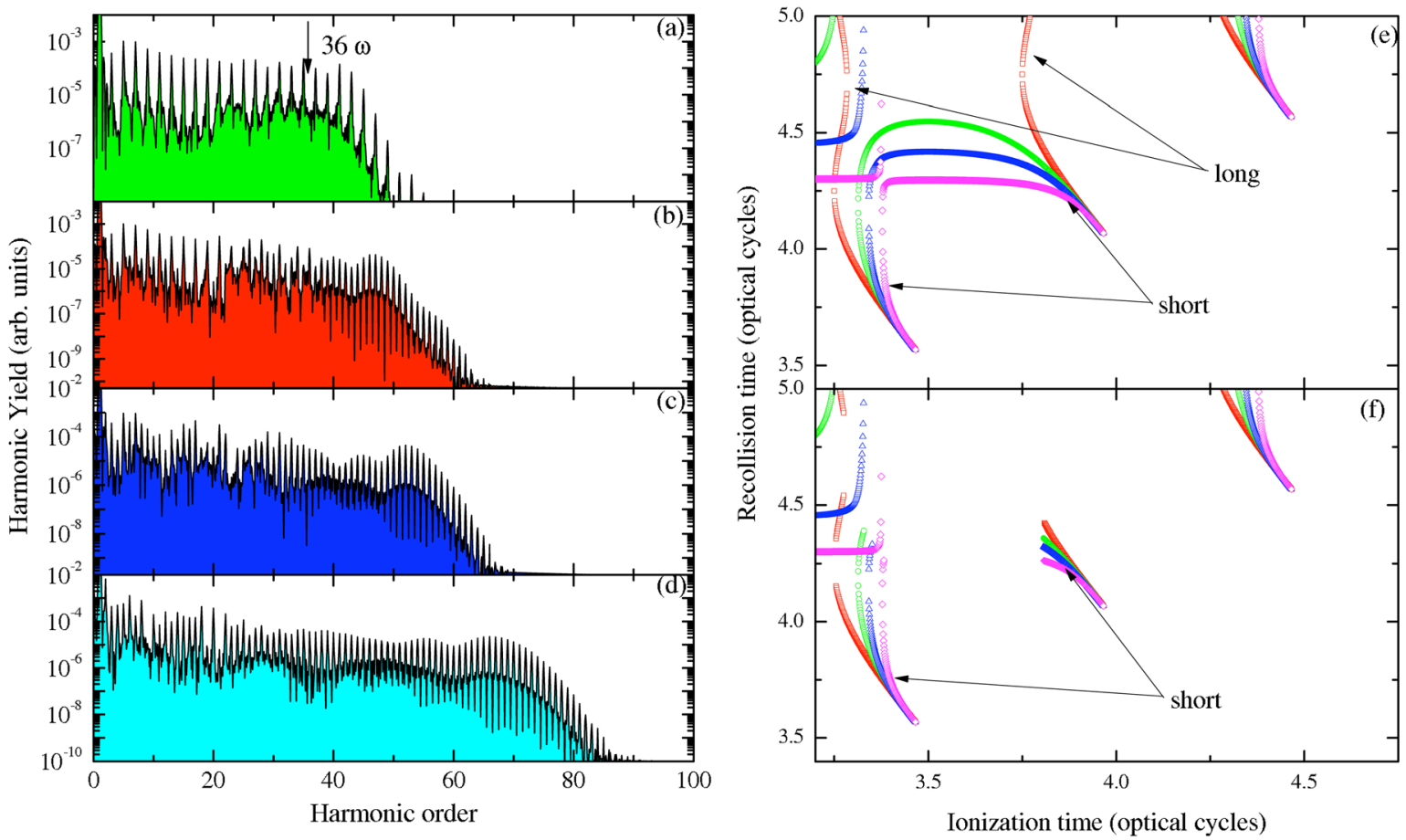

Figure 27. HHG spectra for a model atom with a ground-state energy, $I_{\mathrm{p}}=-0.67$ a.u. obtained using the 1D-TDSE approach. The laser parameters are $I=2 \times 10^{14} \mathrm{~W} \cdot \mathrm{cm}^{-2}$ and $\lambda=800 \mathrm{~nm}$. We have used a trapezoidal shaped pulse with two optical cycles turn on and turn off, and a plateau with six optical cycles, 10 optical cycles in total, i.e. approximately $27 \mathrm{fs}$. The arrow indicates the cutoff predicted by the semiclassical model (Lewenstein et al 1994). Panel (a): homogeneous case, (b): $\varepsilon=0.01$ (100 a.u), (c): $\varepsilon=0.02$ (50 a.u) and (d): $\varepsilon=0.05$ (20 a.u). The numbers in brackets indicate an estimate of the inhomogeneity region (for more details see e.g. Ciappina et al (2012b), Husakou et al (2011a)) . In panels (e) and (f) is shown the dependence of the semiclassical trajectories on the ionization and recollision times for different values of $\varepsilon$ and for the non confined case, panel (e) and the confined case, panel (f), respectively. Red squares: homogeneous case $\varepsilon=0$; green circles: $\varepsilon=0.01$; blue triangles: $\varepsilon=0.02$ and blue triangles: $\varepsilon=0.05$. Figure reused with permission from Ciappina et al (2012b). Copyright 2012 by American Physical Society.
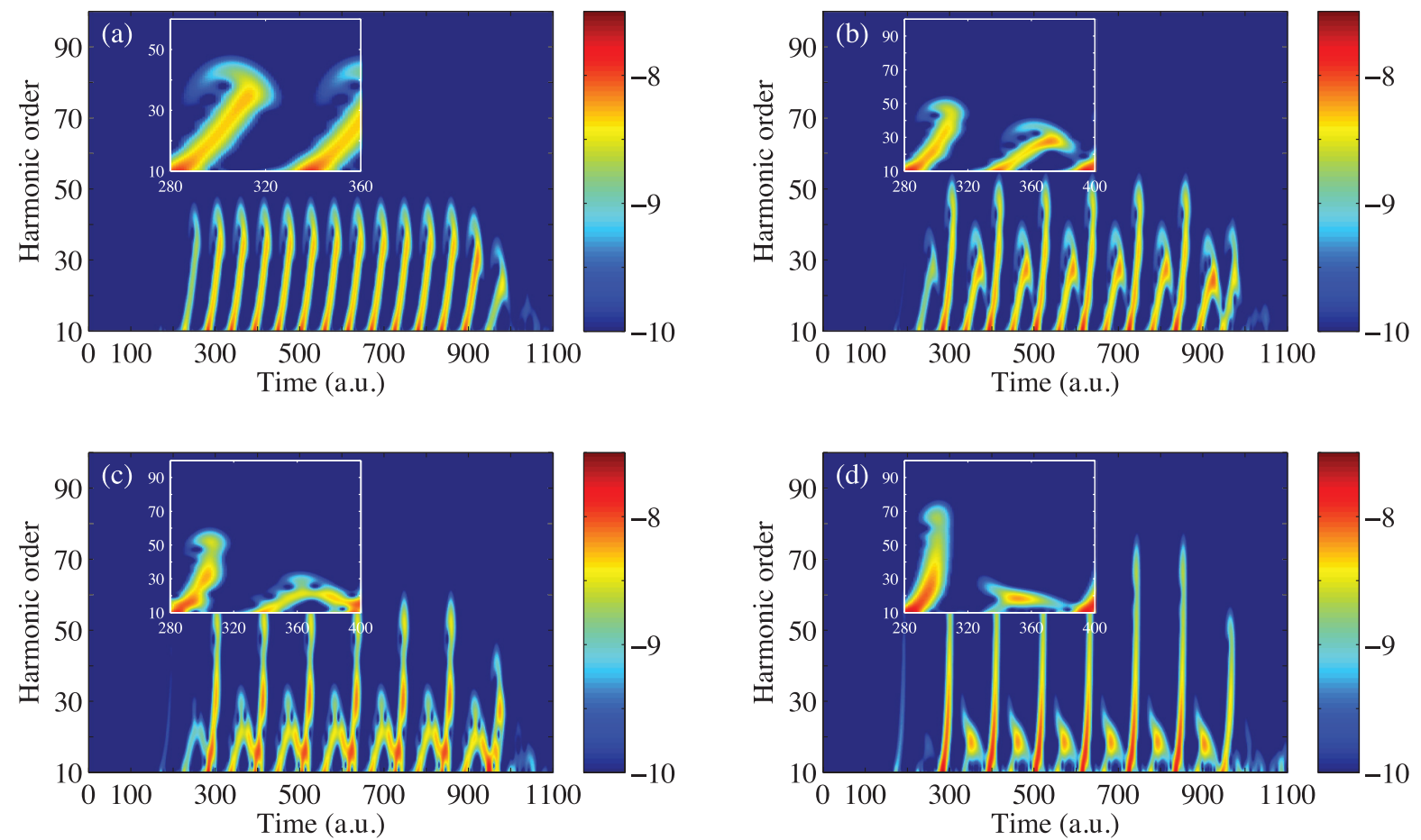

Figure 28. Panels (a)-(d): Gabor analysis for the corresponding HHG spectra of panels (a)-(d) of figure 27. The zoomed regions in all panels show a time interval during the laser pulse for which the complete electron trajectory, from birth time to recollision time, falls within the pulse plateau. In panels (a)-(d) the color scale is logarithmic. Figure reused with permission from Ciappina et al (2012b). Copyright 2012 by American Physical Society. 

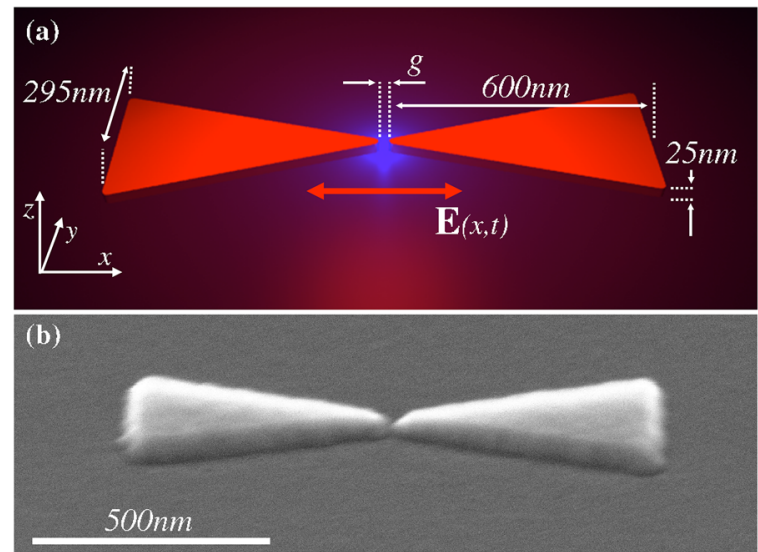

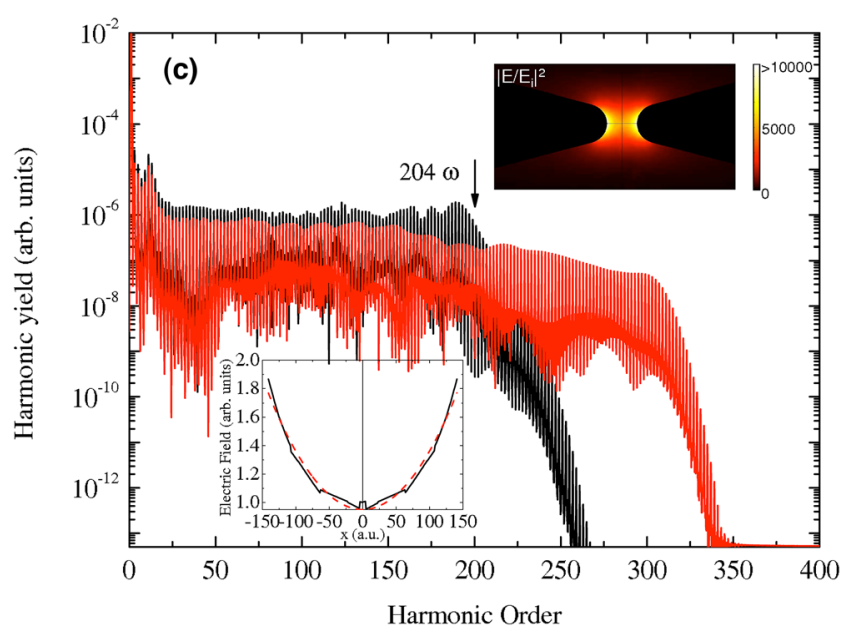

Figure 29. (a) Schematic representation of the geometry of the considered nanostructure. A gold bow-tie antenna resides on glass substrate (refractive index $n=1.52)$ with superstate medium of air $(n=1)$. The characteristic dimensions of the system and the coordinate system used in the 1D-TDSE simulations are shown. (b) SEM image of a real gold bow-tie antenna. (c) HHG spectra for a model of argon atoms $\left(I_{\mathrm{p}}=-0.58\right.$ a.u. $)$, driven by a laser pulse with wavelength $\lambda=1800 \mathrm{~nm}$ and intensity $I=1.25 \times 10^{14} \mathrm{~W} \cdot \mathrm{cm}^{-2}$ at the center of the gap $x=0$. We have used a trapezoidal shaped pulse with three optical cycles turn on and turn off, and a plateau with four optical cycles (about $60 \mathrm{fs}$ ). The gold bow-tie nanostructure has a gap $g=15 \mathrm{~nm}$ (283 a.u.). The black line indicates the homogeneous case while the red line indicates the nonhomogeneous case. The arrow indicates the cutoff predicted by the semiclassical model for the homogeneous case (Lewenstein et al 1994). The top left inset shows the functional form of the electric field $E(x, t)$, where the solid lines are the raw data obtained from the finite element simulations and the dashed line is a nonlinear fitting. The top right inset shows the intensity enhancement in the gap region of the gold bow-tie nanostructure. Figure reused from Ciappina et al (2012a) (CC BY 3.0).

by employing the semiclassical arguments (see panels (e), (f) in figure 27) behind the SFA and time-frequency analysis tools (figure 28), we are able to fully explain the reasons of the cutoff extension.

\subsection{Spatially (linear) nonhomogeneous fields: the SFA approach}

In this section we summarize the work done in Shaaran et al (2012b). In this contribution, we perform a detailed analysis of HHG in atoms within the SFA by considering spatially (linear) inhomogeneous monochromatic laser fields. We investigate how the individual pairs of quantum orbits contribute to the harmonic spectra. To this end we have modified both the classical action and the saddle points equations by including explicitly the spatial dependence of the laser field. We show that in the case of a linear inhomogeneous field the electron tunnels with two different canonical momenta. One of these momenta leads to a higher cutoff and the other one develops a lower cutoff. Furthermore, we demonstrate that the quantum orbits have a very different behavior in comparison to the conventional homogeneous field. A recent study supports our initial findings (Zagoya et al 2016).

We also conclude that in the case of the inhomogeneous fields both odd and even harmonics are present in the HHG spectra. Within our extended SFA model, we show that the HHG cutoff extends far beyond the standard semiclassical cutoff in spatially homogeneous fields. Our findings are in good agreement both with quantum-mechanical and classical models. Furthermore, our approach confirms the versatility of the SFA approach to tackle now the HHG driven by spatially (linear) inhomogeneous fields.

\subsection{Real nonhomogeneous fields}

In this section we present numerical simulations of HHG in an argon model atom produced by the fields generated when a gold bow-tie nanostructure is illuminated by a short laser pulse of long wavelength $\lambda=1800 \mathrm{~nm}$ (see Ciappina et al (2012a) for more details). The functional form of these fields is extracted from finite element simulations using both the complete geometry of the metal nanostructure and laser pulse characteristics (see figure 29(a)). We use the numerical solution of the TDSE in reduced dimensions to predict the HHG spectra. A clear extension in the HHG cutoff position is observed. This characteristic could lead to the production of XUV coherent laser sources and open the avenue to the generation of shorter attosecond pulses. It is shown in figure 29(c) that this new feature is a consequence of the combination of a spatial nonhomogeneous electric field, which modifies substantially the electron trajectories, and the confinement of the electron dynamics. Furthermore, our numerical results are supported by time-analysis and classical simulations. A more pronounced increase in the harmonic cutoff, in addition to an appreciable growth in conversion efficiency, could be attained by optimizing the nanostructure geometry and materials. These degrees of freedom could pave the way to tailor the harmonic spectra according to specific requirements.

\subsection{Temporal and spatial synthesized fields}

In this section we present a brief summary of the results published in Pérez-Hernández et al (2013). In short, numerical simulations of HHG in He atoms using a temporal and spatial synthesized laser field are considered using the full 3D-TDSE. This particular field provides a new route for the 
generation of photons at energies beyond the carbon K-edge using laser pulses at $800 \mathrm{~nm}$, which can be obtained from conventional Ti:Sapphire laser sources. The temporal synthesis is performed using two few-cycle laser pulses delayed in time (Pérez-Hernández et al 2009). On the other hand, the spatial synthesis is obtained by using a spatial nonhomogeneous laser field (Husakou et al 2011a, Ciappina et al 2012b, Yavuz et al 2012) produced when a laser beam is focused in the vicinity of a metal nanostructure or nanoparticle.

Focusing on the spatial synthesis, the nonhomogeneous spatial distribution of the laser electric field can be obtained experimentally by using the resulting field as produced after the interaction of the laser pulse with nanoplasmonic antennas (Kim et al 2008, Husakou et al 2011a, Ciappina et al 2012b, Yavuz et al 2012), metallic nanowaveguides (Park et al 2011), metal (Süßmann and Kling 2011a, Zherebtsov et al 2011) and dielectric nanoparticles (Süßmann and Kling 2011b) or metal nanotips (Hommelhoff et al 2006c, Schenk et al 2010, Krüger et al 2011, 2012a, 2012b, Herink et al 2012).

The coupling between the atom and the laser pulse, linearly polarized along the $z$ axis, is modified in order to treat the spatially nonhomogeneous fields and can be written it as: $V_{1}(z, t, \tau)=\tilde{E}(z, t, \tau) z$ with $\tilde{E}(z, t, \tau)=E(t, \tau)(1+\varepsilon z)$ and $E(t, \tau)=E_{1}(t)+E_{2}(t, \tau)$ the temporal synthesized laser field with $\tau$ the time delay between the two pulses (see e.g. PérezHernández et al (2009) for more details). As in the 1D case the parameter $\varepsilon$ defines the strength of the spatial nonhomogeneity and the dipole approximation is preserved because $\varepsilon \ll 1$.

The linear functional form for the spatial nonhomogeneity described above could be obtained engineering adequately the geometry of plasmonic nanostructures and by adjusting the laser parameters in such a way that the laser-ionized electron feels only a linear spatial variation of the laser electric field when in the continuum (see e.g. Ciappina et al (2012a) and references therein). The harmonic spectrum then obtained in He for $\varepsilon=0.002$ is presented in figure 30(b). We can observe a considerable cut-off extension up to $12.5 U_{p}$ which is much larger when compared with the double pulse configuration employed alone (it leads only to a maximum of $4.5 U_{p}$ (PérezHernández et al 2009)). This large extension of the cutoff is therefore a signature of the combined effect of the double pulse and the spatial nonhomogeneous character of the laser electric field. For this particular value of the laser peak intensity $\left(1.4 \times 10^{15} \mathrm{~W} \mathrm{~cm}^{-2}\right)$ the highest photon energy is greater than $1 \mathrm{keV}$. Note that the quoted intensity is actually the plasmonic enhanced intensity, not the input laser intensity. The latter could be several orders of magnitude smaller, according to the plasmonic enhancement factor (see e.g. Kim et al (2008), Park et al (2011)) and will allow the nanoplasmonic target to survive to the interaction. In order to confirm the underlying physics highlighted by the classical trajectories analysis, we have retrieved the time-frequency distribution of the calculated dipole (from the 3D-TDSE) corresponding to the case of the spectra presented in figure 30(b) using a wavelet analysis. The result is presented in figure 30(a) where we have superimposed the calculated classical recombination energies (in brown) to show the excellent agreement between the two theoretical approaches. The consistency of
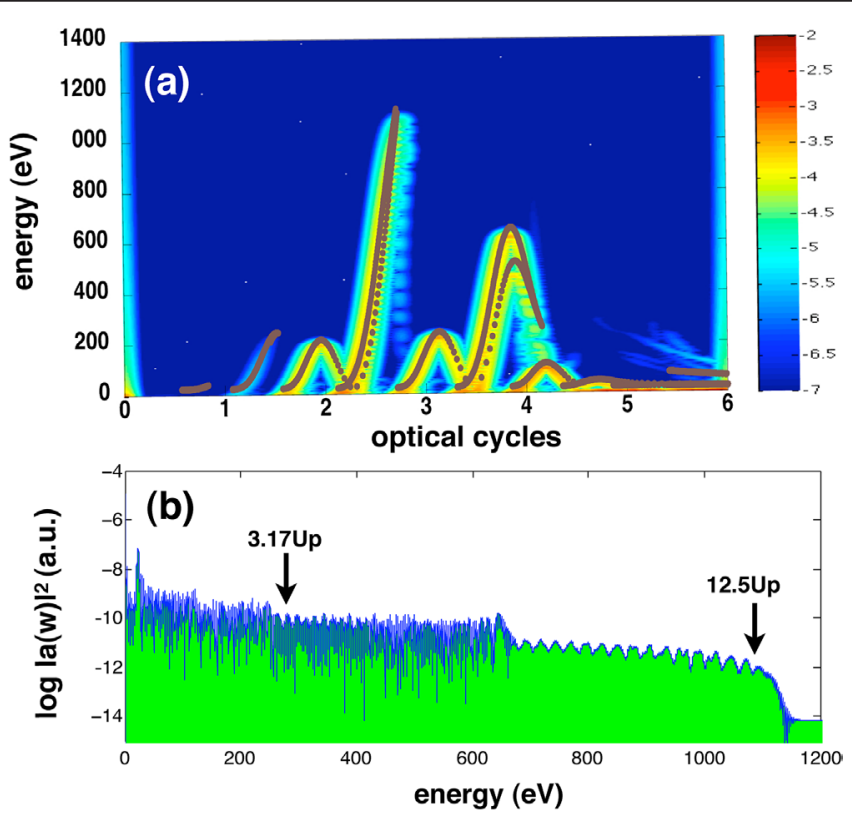

Figure 30. (a) Time-frequency analysis obtained from the 3D-TDSE HHG spectrum for a He atom driven by the spatially and temporally synthesized pulse described in the text with $\varepsilon=0.002$. The plasmonic enhanced intensity $I=1.4 \times 10^{15} \mathrm{~W}$ $\mathrm{cm}^{-2}$. Superimposed (in brown) are the classical rescattering energies; (b) 3D-TDSE HHG spectrum for the same parameters used in (a). Figure reused with permission from Pérez-Hernández et al (2013) and modified. Copyright 2013 by American Physical Society.

the classical calculations with the full quantum approach is clear and confirms the mechanism of the generation of this $12.5 U_{p}$ cut-off extension. In addition, the HHG spectra exhibit a clean continuum as a result of the trajectory selection on the recombination time, which itself is a consequence of employing a combination of temporally and spatially synthesized laser field.

\subsection{Plasmonic near-fields}

This section includes an overview of the results reported in Shaaran et al (2013a). In this contribution it is shown how the HHG spectra from model Xe atoms are modified by using a plasmonic-enhanced near-field generated when a metal nanoparticle is illuminated by a short laser pulse. A setup combining a noble gas as a driven media and metal nanoparticles was also proposed recently in Husakou and Herrmann (2014), Husakou et al (2015).

For our near-field we use the function given by Süßmann and Kling (2011a) to define the spatial nonhomogeneous laser electric field $E(x, t)$, i.e.

$$
E(x, t)=E_{0} f(t) \exp (-x / \chi) \sin \left(\omega_{0} t+\phi_{\mathrm{CEP}}\right) .
$$

The functional form of the resulting laser electric field is extracted from attosecond streaking experiments and incorporated both in our quantum and classical approaches. In this specific case the spatial dependence of the plasmonic nearfield is given by $\exp (-x / \chi)$ and it is a function of both the size and the material of the spherical nanoparticle. $E(x, t)$ is valid 

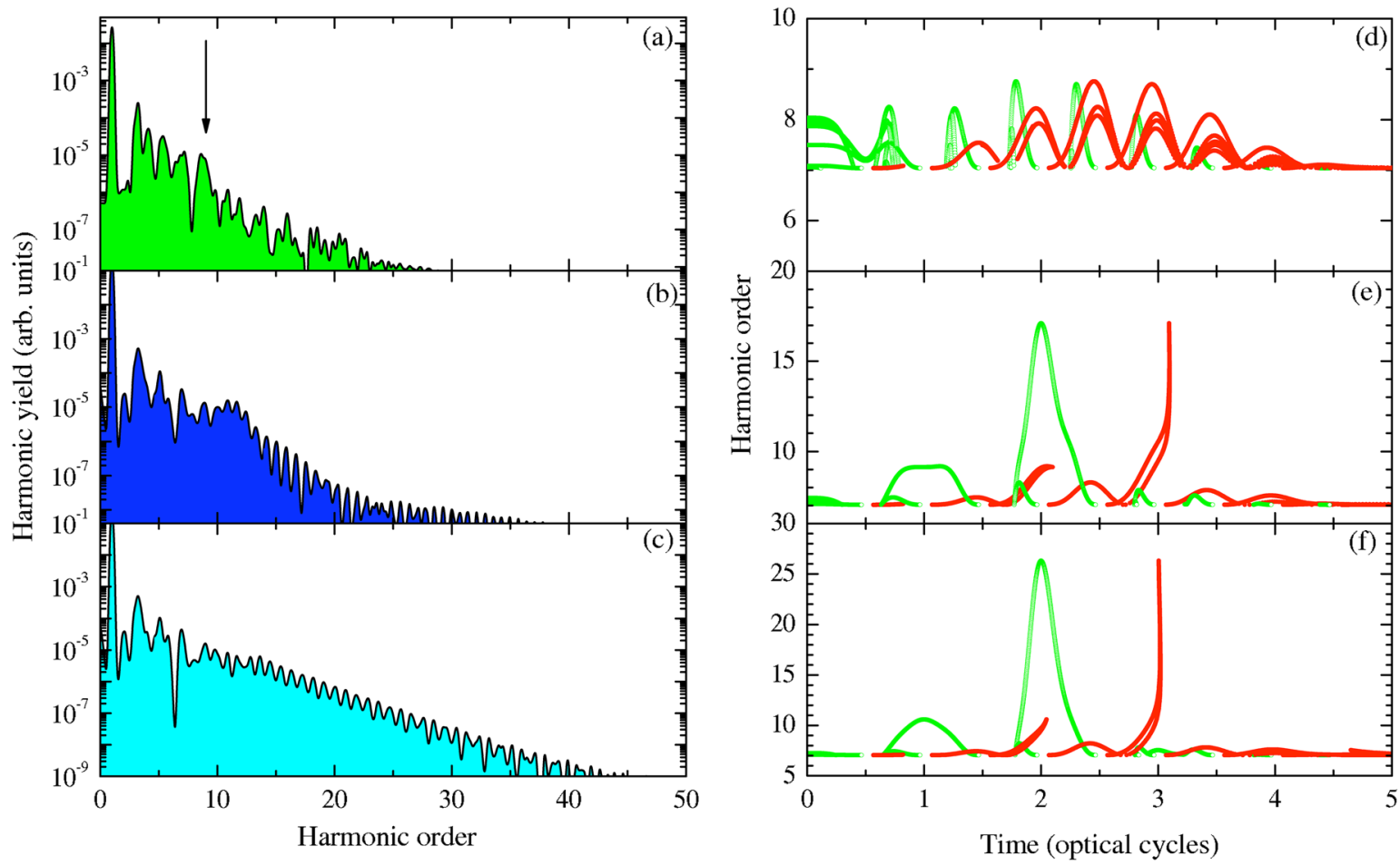

Figure 31. HHG spectra for model Xe atoms, laser wavelength $\lambda=720 \mathrm{~nm}$ and intensity $I=2 \times 10^{13} \mathrm{~W} \cdot \mathrm{cm}^{-2}$. We use a $\sin ^{2}$ pulse envelope with $n=5$. Panel (a) represents the homogeneous case, panel (b) $\chi=50$ and panel (c) $\chi=40$. The arrow in panel (a) indicates the cutoff predicted by the semiclassical approach (Lewenstein et al 1994). Panels (d)-(f) show the corresponding total energy of the electron (expressed in harmonic order) driven by the laser field calculated from Newton-Lorentz equation and plotted as a function of the $t_{i}$ (green (light gray) circles) or the $t_{r}$ (red (dark gray) circles). Figure reused with permission from Shaaran et al (2013a). Copyright 2013 by American Physical Society.

for $x$ outside of the metal nanoparticle, i.e. $x \geqslant R_{0}$, where $R_{0}$ is its radius. It is important to note that the electron motion takes place in the region $x \geqslant R_{0}$ with $\left(x+R_{0}\right) \gg 0$. We consider the laser field having a $\sin ^{2}$ envelope: $f(t)=\sin ^{2}\left(\frac{\omega_{0} t}{2 n_{p}}\right)$, where $n_{p}$ is the total number of optical cycles, i.e. the total pulse duration is $\tau_{L}=2 \pi n_{p} / \omega_{0}$. The harmonic yield of the atom is obtained by Fourier transforming the acceleration $a(t)$ of the electronic wavepacket (see section 7.2).

Figure 31, panels (a)-(c) show the HHG spectra for model xenon atoms generated by a laser pulse with $I=2 \times 10^{13} \mathrm{~W}$ $\mathrm{cm}^{-2}, \lambda=720 \mathrm{~nm}$ and a $\tau_{L}=13$ fs, i.e. $n_{p}=5$ (which corresponds to an intensity envelope of $\approx 4.7 \mathrm{fs}$ FWHM) (Süßmann and Kling 2011a). In the case of a spatial homogeneous field, no harmonics beyond the 9 th order are observed. The spatial decay parameter $\chi$ accounts for the spatial nonhomogeneity induced by the nanoparticle and it varies together with its size and the kind of metal employed. Varying the value of $\chi$ is therefore equivalent to choosing the type of nanoparticle used, which allows to overcome the semiclassically predicted cutoff limit and reach higher harmonic orders. For example, with $\chi=40$ and $\chi=50$ harmonics in the mid 20s (panel (c)) and well above the 9 th order (a clear cutoff at $n_{c} \approx 15$ is achieved) (panel (b)), respectively, are obtained. A modification in the harmonic periodicity, related to the breaking of symmetry imposed by the induced spatial nonhomogeneity, is also clearly noticeable.

Now, by the semiclassical simple man's (SM) model (Corkum 1993, Lewenstein et al 1994) we will study the
HHG cutoff extension. This new effect may be caused by a combination of several factors (for details see Ciappina et al $(2012 \mathrm{a}, 2012 \mathrm{~b}))$. As is well known, the cutoff law is $n_{c}=\left(3.17 U_{p}+I_{p}\right) / \omega_{0}$, where $n_{c}$ is the harmonic order at the cutoff and $U_{p}$ the ponderomotive energy. We solve numerically equation (19) for an electron moving in an electric field with the same parameters used in the 1D-TDSE calculations, i.e.

$$
\ddot{x}(t)=-\nabla_{x} V_{l}(x, t)=-E(x, t)\left(1-\frac{x(t)}{\chi}\right),
$$

and consider the SM model initial conditions: the electron starts at position zero at $t=t_{i}$ (the ionization time) with zero velocity, i.e. $x\left(t_{i}\right)=0$ and $\dot{x}\left(t_{i}\right)=0$. When the electric field reverses, the electron returns to its initial position (i.e. the electron recollides or recombines with the parent ion) at a later time $t=t_{r}$ (the recombination time), i.e. $x\left(t_{r}\right)=0$. The electron kinetic energy at the $t_{r}$ is calculated as usual from: $E_{k}\left(t_{r}\right)=\frac{\dot{x}\left(t_{r}\right)^{2}}{2}$ and finding the $t_{r}$ (as a function of $t_{i}$ ) that maximizes $E_{k}, n_{c}$ is also maximized.

Panels (d)-(f) of figure 31 represent the behaviour of the harmonic order upon the $t_{i}$ and $t_{r}$, calculated from $n=\left(E_{k}\left(t_{i, r}\right)+I_{p}\right) / \omega$ as for the cases (a)-(c) of figure 31 , respectively. Panels (e) and (f) show how the nonhomogeneous character of the laser field strongly modifies the electron trajectories towards an extension of the $n_{c}$. This is clearly present at $n_{c} \sim 18 \omega(28 \mathrm{eV})$ and $n_{c} \sim 27 \omega(42 \mathrm{eV})$ for $\chi=50$ 
and $\chi=40$, respectively. These last two cutoff extensions are consistent with the quantum predictions presented in panels (b) and (c) of figure 31.

Classical and quantum approaches predict cutoff extensions that could lead to the production of XUV coherent laser sources and open a direct route to the generation of attosecond pulses. This effect is caused by the induced laser field spatial nonhomogeneity, which modifies substantially the electron trajectories. A more pronounced increment in the harmonic cutoff, in addition to an appreciable growth in the conversion efficiency, could be reached by varying both the radius and the metal material of the spherical nanoparticles. These new degrees of freedom could pave the way to extend the harmonic plateau reaching the XUV regime with modest input laser intensities.

\subsection{Metal nanotip photoemission}

In all the preceding sections we use plasmonic enhanced fields as sources and atoms as active media. On the contrary, in this section we predict that it is entirely possible to generate high-order harmonic radiation directly from metal nanotips. By employing available laser source parameters and treating the metal tip with a fully quantum mechanical model within the SAE approximation, we are able to model the HHG process using a metal as active medium. As in previous cases we do not take into account any collective effect, such as propagation and phase matching. Arguably, such collective effects could play a minor role in the generation of coherent radiation using nanosources due to the fact that radiation emission occurs at a sub-wavelength scale (see, e.g. Kim et al (2008)). As was already discussed, the main physical mechanism behind the generation of high-order harmonics is the electron recollision step and consequently any reliable model should include it. It was already shown that the recollision mechanism is also needed to describe ATP measurements and, considering these two laser-matter phenomena, i.e. the photoemitted electrons and the high-frequency radiation, are physically linked, we could conclude that metal nanotips can be used as sources of coherent XUV radiation as well. The theoretical model we use in this case has already been described in previous sections and employed for the calculation of electron photoemission from metal nanotips (Hommelhoff et al 2006c, Krüger et al 2012a). As a consequence we do not repeat it here (for details we refer the reader to Ciappina et al (2014c)) and we only show and discuss briefly a couple of typical results.

In figure 32, we show HHG spectra by using a long (tencycle) trapezoidal (two cycles of turn on and off and six cycles of constant amplitude, 23 fs of total time) laser pulse of $\lambda=685 \mathrm{~nm}$ (the corresponding photon energy is $1.81 \mathrm{eV}$ ). The different panels correspond to a set of values of the peak laser electric field $E_{0}$, namely, 10,15 , and $20 \mathrm{GV} \mathrm{m}^{-1}$ for figures 32(a)-(c), respectively. For the three cases we have employed two values for the DC field, $E_{\mathrm{dc}}$ : $-0.4 \mathrm{GV} \mathrm{m}^{-1}$ (blue -dark gray) and $2 \mathrm{GV} \mathrm{m}^{-1}$ (cyan -light gray). Two main features can be observed: (i) an increase of the relative yield in the plateau region for positive values of the $E_{\mathrm{dc}}$ field. This

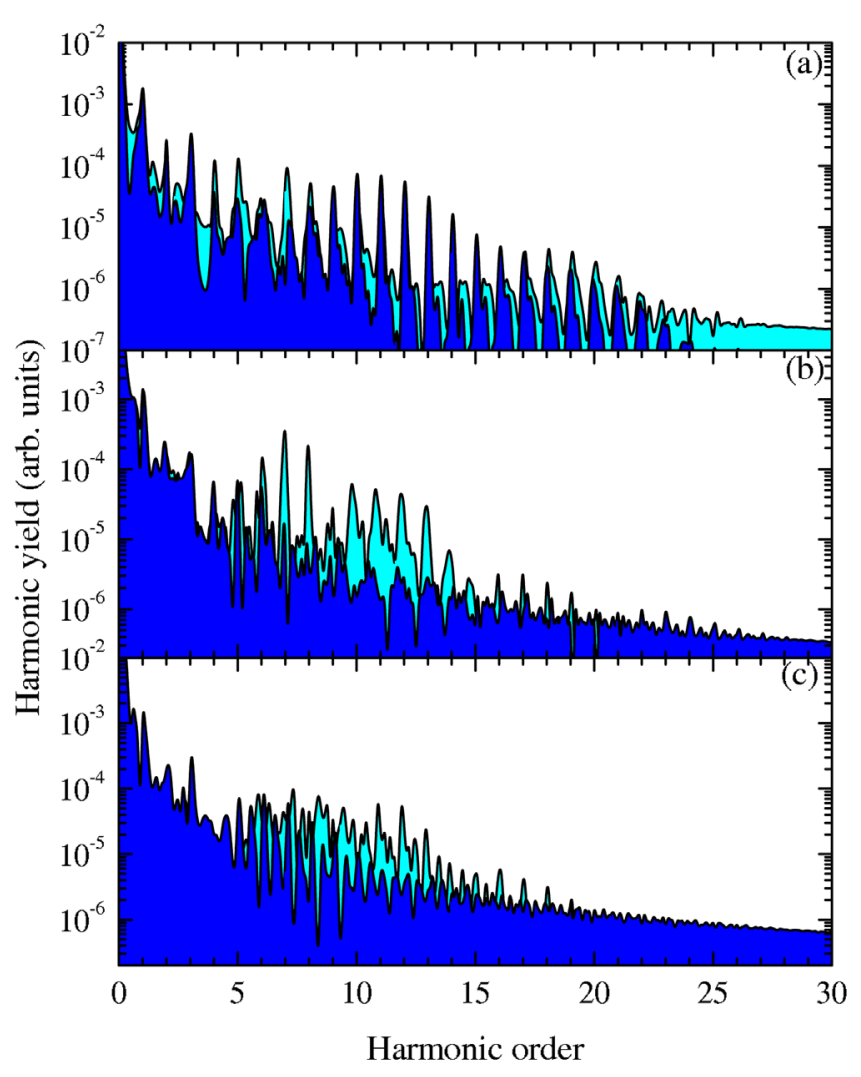

Figure 32. Plots of the HHG spectra as a function of harmonic order for a metal $(\mathrm{Au})$ nanotip using a trapezoidal shaped laser pulse with ten cycles of total time, $\lambda=685 \mathrm{~nm}$, and (a) $E_{0}=$ $10 \mathrm{GV} \mathrm{m}^{-1}$, (b) $E_{0}=15 \mathrm{GV} \mathrm{m}^{-1}$, and (c) $E_{0}=20 \mathrm{GV} \mathrm{m}^{-1}$. In all panels blue denotes $E_{\mathrm{dc}}=-0.4 \mathrm{GV} \mathrm{m}^{-1}$ and cyan $E_{\mathrm{dc}}=2$ $\mathrm{GV} \mathrm{m}{ }^{-1}$. Note that the harmonic yield scale ( $y$ axis) is different in each panel. Figure reused with permission from Ciappina et al (2014c). Copyright 2014 by American Physical Society.

gain in conversion efficiency is important for ease of experimental radiation detection; (ii) the occurrence of odd and even harmonics (see, e.g. figure 32(a)), which is due to the broken symmetry at the metal surface of the nanotip, in contrast to an atomic gas, which represents a typical centrosymmetric nonlinear medium.

The main result of this section is that we show it is possible to generate high-order harmonics directly from metal nanotips. Our predictions are based on a quantum mechanical approach, already successfully applied to model the photoelectron spectra under similar experimental conditions. As a consequence it appears perfectly feasible to obtain coherent harmonic radiation directly from these metal nanosources.

\section{ATI driven by spatially inhomogeneous fields}

As was mentioned at the outset, ATI represents another key strong field phenomena. As a consequence, in the next sections we summarize the theoretical work we have done in order to tackle the ATI driven by spatially inhomogeneous fields. As in the case of $\mathrm{HHG}$, we include here results obtained using quantum, semiclassical and classical formalisms. 


\section{1. $1 D$ case}

Investigations carried out on ATI, generated by few-cycle driving laser pulses, have attracted much interest due to the sensitivity of the energy and angle-resolved photoelectron spectra to the absolute value of the CEP (Milošević et al 2006, Sayler et al 2011). This feature makes the ATI phenomenon a potential tool for laser pulse characterization. In order to characterize the CEP of a few-cycle laser pulse, the so-called backward-forward asymmetry of the ATI spectrum is measured and from the information collected the absolute CEP can be obtained (Paulus et al 2001, Sayler et al 2011). Furthermore, nothing but the high energy region of the photoelectron spectrum appears to be strongly sensitive to the absolute CEP and consequently electrons with high kinetic energy are needed in order to characterize it (Paulus et al 2001, 2003, Milošević et al 2006).

Nowadays, experiments have demonstrated that the electron spectra of ATI could be extended further by using plasmon field enhancement (Kim et al 2008, Zherebtsov et al 2011). The strong confinement of the plasmonics spots and the distortion of the electric field by the surface plasmons induces a spatial inhomogeneity in the driving laser field, just before the interaction with the corresponding target gas. A related process employing solid state targets instead of atoms and molecules in gas phase is the so called above-threshold photoemission (ATP). This laser driven phenomenon has received special attention recently due to its novelty and the new physics involved. In ATP electrons are emitted directly from metallic surfaces or metal nanotips and they present distinct characteristics, namely higher energies, far beyond the usual cutoff for noble gases and consequently the possibility to reach similar electron energies with smaller laser intensities (see e.g. Herink et al (2012), Hommelhoff et al (2006c), Krüger et al (2014), Krüger et al (2011), Schenk et al (2010)). Furthermore, the photoelectrons emitted from these nanosources are sensitive to the CEP and consequently it plays an important role in the angle and energy resolved photoelectron spectra (Apolonski et al 2004, Krüger et al 2011, Zherebtsov et al 2011).

Despite new developments, all numerical and semiclassical approaches to model the ATI phenomenon are based on the assumption that the external field is spatially homogeneous in the region where the electron dynamics takes place. For a spatially inhomogeneous field, however, important modifications will occur to the strong field phenomena, as was already shown for the case of HHG. These modifications occur because the laser-driven electric field, and consequently the force applied on the electron, will also depend on its position.

From a theoretical viewpoint, the ATI process can be tackled using different approaches (for a summary see e.g. Milošević et al (2006) and references therein). In this section, we concentrate on extending one of the most and widely used approaches: the numerical solution of the TDSE in reduced dimensions.

In order to calculate the energy-resolved photoelectron spectra, we use the same 1D-TDSE employed for the computation of HHG (see section 7.2). For calculating the energyresolved photoelectron spectra $P(E)$ we use the window function technique developed by Schafer (1991), Schafer and
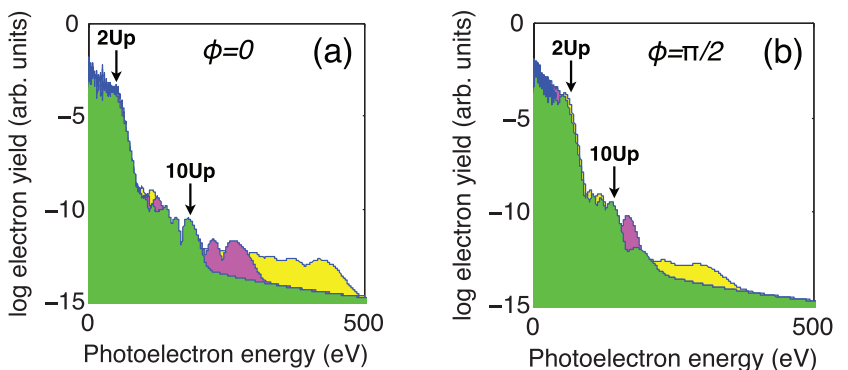

Figure 33. 1D-TDSE energy-resolved photoelectron spectra for a model atom with $I_{p}=-0.5$ a.u. and for the laser parameters, $I=3 \times 10^{14} \mathrm{~W} \mathrm{~cm}^{-2}, \lambda=800 \mathrm{~nm}$ and a sin-squared shaped pulse with a total duration of 4 cycles (10 fs). In green for $\varepsilon=0$ (homogeneous case), in magenta for $\varepsilon=0.003$ and in yellow for $\varepsilon=0.005$. Panel (a) represent the case for $\phi_{\mathrm{CEP}}=0$ (sin-like pulse) and panel (b) represents the case for $\phi_{\mathrm{CEP}}=\pi / 2$ (cos-like pulse). The arrows indicate the $2 U_{p}$ and $10 U_{p}$ cutoffs predicted by the classical model (Milošević et al 2006). Figure reused with permission from Ciappina et al (2012) and modified. Copyright 2012 by American Physical Society.

Kulander (1990). This tool has been widely used, both to calculate $P(E)$ and angle-resolved photoelectron spectra (see section 9.2) (Schafer 2009) and it represents a step forward with respect to the usual projection methods.

In our simulations we employ as a driving field a four-cycle (total duration $10 \mathrm{fs}$ ) sin-squared laser pulse with an intensity $I=3 \times 10^{14} \mathrm{~W} \mathrm{~cm}^{-2}$ and wavelength $\lambda=800 \mathrm{~nm}$. We chose a spatially linear inhomogeneous field and three different values for the parameter that characterizes the inhomogeneity strength, namely $\varepsilon=0$ (homogeneous case), $\varepsilon=0.003$ and $\varepsilon=0.005$. Figure 33(a) shows the cases with $\phi_{\mathrm{CEP}}=0$ (a sin-like laser pulse) meanwhile in figure 33(b) $\phi_{\mathrm{CEP}}=\pi / 2$ (a cos-like laser pulse), respectively. In both panels green represents the homogeneous case, i.e. $\varepsilon=0$, magenta is for $\varepsilon=0.003$ and yellow is for $\varepsilon=0.005$, respectively. For the homogeneous case, the spectra exhibits the usual distinct behavior, namely the $2 U_{p}$ cutoff ( $\approx 36 \mathrm{eV}$ for our case) and the $10 U_{p}$ cutoff $(\approx 180 \mathrm{eV})$, where $U_{p}=E_{0}^{2} / 4 \omega^{2}$ is the ponderomotive potential. The former cutoff corresponds to those electrons that, once ionized, never return to the atomic core, while the latter one corresponds to the electrons that, once ionized, return to the core and elastically rescatter. It is well established using classical arguments that the maximum kinetic energies of the direct and the rescattered electrons are $E_{\max }^{d}=2 U_{p}$ and $E_{\max }^{r}=10 U_{p}$, respectively. In a quantum mechanical approach, however, it is possible to find electrons with energies beyond the $10 U_{p}$, although their yield drops several orders of magnitude (Milošević et al 2006). The TDSE, which can be considered as an exact approach to the problem, is able to predict the $P(E)$ for the whole range of electron energies. In addition, the most energetic electrons, i.e. those with $E_{k} \gg 2 U_{p}$, are used to characterize the CEP of few-cycle pulses. As a result, a correct description of the rescattering mechanism is needed.

For the spatial inhomogeneous case, the cutoff positions of both the direct and the rescattered electrons are extended 
towards larger energies. For the rescattered electrons, this extension is very prominent. In fact, for $\varepsilon=0.003$ and $\varepsilon=0.005$, it reaches $\approx 260 \mathrm{eV}$ and $\approx 420 \mathrm{eV}$, respectively (see figure 33(a)). Furthermore, it appears that the high energy region of $P(E)$, for instance, the region between $200-400 \mathrm{eV}$ for $\varepsilon=0.005$ (figure 33 in yellow), is strongly sensitive to the CEP. This feature indicates that the high energy region of the photoelectron spectra could resemble a new and better CEP characterization tool. It should be, however, complemented by other well known and established CEP characterization tools, as, for instance, the forward-backward asymmetry (see Milošević et al (2006)). Furthermore, the utilization of nonhomogeneous fields would open the avenue for the production of high energy electrons, reaching the $\mathrm{keV}$ regime, if a reliable control of the spatial and temporal shape of the laser electric field is attained.

We now concentrate our efforts on explaining the extension of the energy-resolved photoelectron spectra using classical arguments. From the SM model (Corkum 1993, Lewenstein et al 1994) we can describe the physical origin of the ATI process as follows: an atomic electron at a position $x=0$, is released or born at a given time, that we call ionization time $t_{i}$, with zero velocity, i.e. $\dot{x}\left(t_{i}\right)=0$. This electron now moves solely under the influence of the oscillating laser electric field (the residual Coulomb interaction is neglected in this model) and will reach the detector either directly or through a rescattering process. By using the classical equation of motion, it is possible to calculate the maximum energy of the electron for both direct and rescattered processes. The Newton equation of motion for the electron in the laser field can be written as (see equation (19)):

$$
\begin{aligned}
\ddot{x}(t) & =-\nabla_{x} V_{1}(x, t) \\
& =E(x, t)+\left[\nabla_{x} E(x, t)\right] x \\
& =E(t)(1+2 \varepsilon x(t)),
\end{aligned}
$$

where we have collected the time dependent part of the electric field in $E(t)$, i.e. $E(t)=E_{0} f(t) \sin \left(\omega t+\phi_{\mathrm{CEP}}\right)$ and specialized to the case $h(x)=x$. In the limit where $\varepsilon=0$ in equation (22), we recover the conventional (spatially homogeneous) case. For the direct ionization, the kinetic energy of an electron released or born at time $t_{i}$ is

$$
E_{d}=\frac{\left[\dot{x}\left(t_{i}\right)-\dot{x}\left(t_{f}\right)\right]^{2}}{2}
$$

where $t_{f}$ is the end time of the laser pulse. For the rescattering process, in which the electron returns to the core at a time $t_{r}$ and reverses its direction, the kinetic energy of the electron yields

$$
E_{r}=\frac{\left[\dot{x}\left(t_{i}\right)+\dot{x}\left(t_{f}\right)-2 \dot{x}\left(t_{r}\right)\right]^{2}}{2} .
$$

For homogeneous fields, equations (23) and (24) become $E_{d}=\frac{\left[A\left(t_{i}\right)-A\left(t_{f}\right)\right]^{2}}{2}$ and $E_{r}=\frac{\left[A\left(t_{i}\right)+A\left(t_{f}\right)-2 A\left(t_{r}\right)\right]^{2}}{2}$, with $A(t)$ being the laser vector potential $A(t)=-\int^{t} E\left(t^{\prime}\right) \mathrm{d} t^{\prime}$. For the case with $\varepsilon=0$, it can be shown that the maximum value for $E_{d}$ is $2 U_{p}$ while for $E_{r}$ it is $10 U_{p}$ (Milošević et al 2006). These two values appear as cutoffs in the energy resolved photoelectron

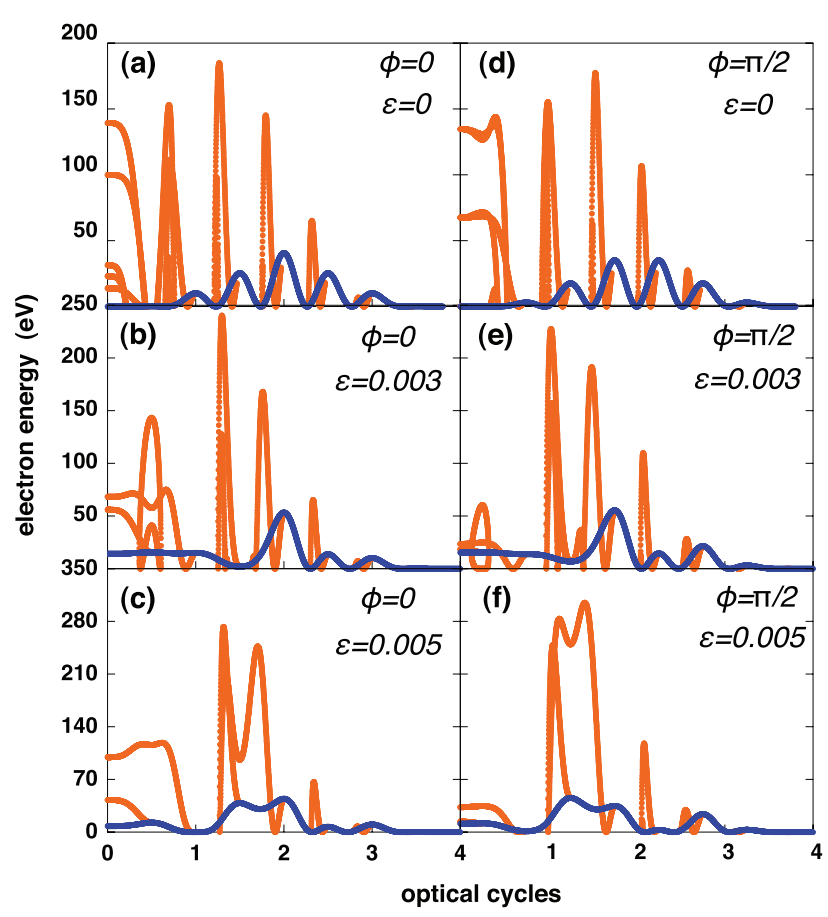

Figure 34. Numerical solutions of equation (22) plotted in terms of the direct (blue) and rescattered (orange) electron kinetic energy. The laser parameters are the same as in figure 33. Panels (a)-(c) correspond to the case of sin-like pulses $\left(\phi_{\mathrm{CEP}}=0\right)$ and for $\varepsilon=0$ (homogeneous case), $\varepsilon=0.003$ and $\varepsilon=0.005$, respectively. Panels (d)-(f) correspond to the case of cos-like pulses $\left(\phi_{\mathrm{CEP}}=\pi / 2\right)$ and for $\varepsilon=0$ (homogeneous case), $\varepsilon=0.003$ and $\varepsilon=0.005$, respectively. Figure reused with permission from Ciappina et al (2012) and modified. Copyright 2012 by American Physical Society.

spectrum as can be observed in panels (a) and (b), in green, in figure 33 (see the respective arrows).

In figure 34 , we present the numerical solutions of equation (22), which is plotted in terms of the kinetic energy of the direct and rescattered electrons. We employ the same laser parameters as in figure 33. Panels (a)-(c) correspond to the case of $\phi_{\mathrm{CEP}}=0$ (sin-like pulses) and for $\varepsilon=0$ (homogeneous case), $\varepsilon=0.003$ and $\varepsilon=0.005$, respectively. Meanwhile, panels (d)-(f) correspond to the case of $\phi_{\mathrm{CEP}}=\pi / 2$ (cos-like pulses) and for $\varepsilon=0$ (homogeneous case), $\varepsilon=0.003$ and $\varepsilon=0.005$, respectively. From the panels (b), (c), (e) and (f) we can observe the strong modifications that the spatial nonhomogeneous character of the laser electric field produces in the electron kinetic energy. These are related to the changes in the electron trajectories (for details see e.g. Ciappina et al (2012a, 2012b), Yavuz et al (2012)). In short, the electron trajectories are modified in such a way that now the electron ionizes at an earlier time and recombines later, and in this way it spends more time in the continuum acquiring energy from the laser electric field. Consequently, higher values of the kinetic energy are attained. A similar behavior with the photoelectrons was observed recently in ATP using metal nanotips. According to the model presented in Herink et al (2012) the localized fields modify the electron motion in such a way to allow sub-cycle dynamics. In our studies, however, we consider both direct and rescattered electrons 
(in Herink et al (2012) only direct electrons are modeled) and the characterization of the dynamics of the photoelectrons is more complex. Nevertheless, the higher kinetic energy of the rescattered electrons is a clear consequence of the strong modifications of the laser electric field in the region where the electron dynamics takes place, as in the above mentioned case of ATP.

\section{2. $3 D$ case}

The logical extension to the numerical approach presented in the previous section is to use the 3D-TDSE of section 7.2 to calculate angular electron momentum distributions and photoelectron spectra of atoms driven by spatially inhomogeneous fields. As in the 1D case the nonhomogeneous character of the laser electric field plays an important role on the ATI phenomenon. In addition, our 3D approach allows us to model in a reliable way the ATI process both in the tunneling and multiphoton regimes. We show that for the former, the spatial nonhomogeneous field causes significant modifications on the electron momentum distributions and photoelectron spectra, while its effects in the later appear to be negligible. Indeed, through the tunneling ATI process, one can obtain higher energy electrons as well as a high degree of asymmetry in the momentum space map. In our study we consider near infrared laser fields with intensities in the mid- $10^{14}$ $\mathrm{W} \mathrm{cm} \mathrm{cm}^{-2}$ range. We use a linear approximation for the plasmonic field, considered valid when the electron excursion is small compared with the inhomogeneity region. Indeed, our 3D simulations confirm that plasmonic fields could drive electrons with energies in the near-keV regime (see e.g. Ciappina et al (2013a)).

In order to obtain a more complete description of the ATI phenomenon driven by spatially nonhomogeneous fields, we solve the 3D-TDSE in the length gauge (see section 7.2). We then investigate the electron momentum distribution and energy-resolved photoelectron spectra $P(E)$, including the dynamics of both, direct and rescattered electrons.

As in the $1 \mathrm{D}$ case, the ATI spectrum is calculated starting from the time propagated electron wave function, once the laser pulse has ceased. As a preliminary test and in order to assure the consistency of our numerical simulations, we have compared our calculations with the results previously obtained in Schafer (1991). The comparison confirms the high degree of accuracy of our implementation as shown in figure 35. For computing the energy-resolved photoelectron spectra $P(E)$ and two-dimensional electron distributions $\mathcal{H}\left(k_{z}, k_{r}\right)$, where $k_{z}\left(k_{r}\right)$ is the electron momentum component parallel (perpendicular) to the polarization direction, we use the window function approach developed in Schafer (1991), Schafer and Kulander (1990).

Experimentally speaking, both the direct and rescattered electrons contribute to the energy-resolved photoelectron spectra. It means that for tackling this problem both physical mechanisms should to be included in any theoretical model. In that sense, the 3D-TDSE, which can be considered as an exact approach to the ATI problem for atoms and molecules in the SAE approximation, appears to be the most adequate tool

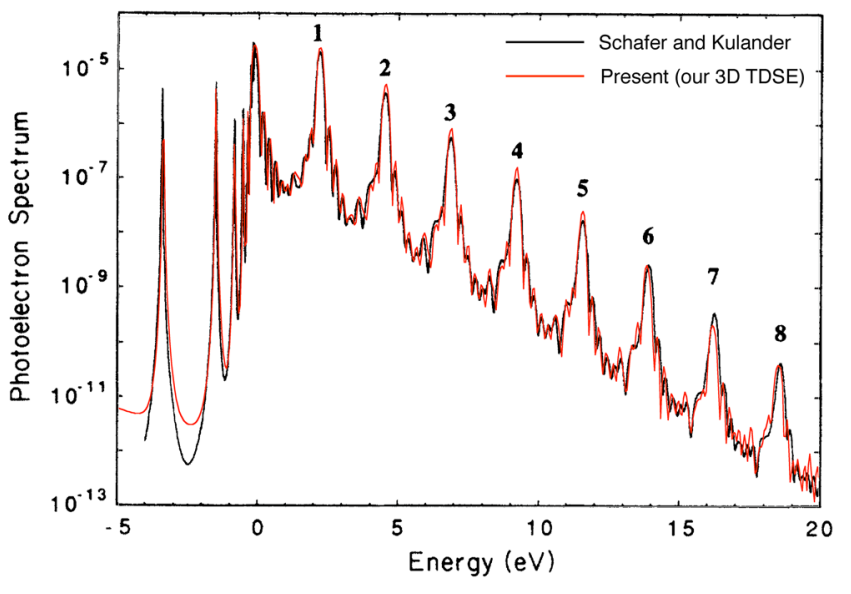

Figure 35. Photoelectron spectrum resulting from our 3D TDSE simulations (in red) and superimposed (in black) with the ATI results calculated by Schafer and Kulander in Schafer (1991). The laser wavelength is $\lambda=532 \mathrm{~nm}$ and the intensity is $I=2 \times 10^{13}$ $\mathrm{W} \mathrm{cm}{ }^{-2}$ (see figure 1 in Schafer (1991) for more details. Figure reused with permission from Ciappina et al (2013a). Copyright 2013 by American Physical Society.

both to predict $P(E)$ and $\mathcal{H}\left(k_{z}, k_{r}\right)$ in the whole range of electron energies and momentum values and angles, respectively.

In the following, we calculate two-dimensional electron momentum distributions for a laser field intensity of $I=5.0544 \times 10^{14} \mathrm{~W} \mathrm{~cm}^{-2}\left(E_{0}=0.12\right.$ a.u $)$. The results are depicted in figure 36 for $\phi_{\mathrm{CEP}}=\pi / 2$. Here, panels (a)-(d) represent the cases with $\varepsilon=0$ (homogeneous case), $\varepsilon=0.002$, $\varepsilon=0.003$ and $\varepsilon=0.005$, respectively. By a simple inspection of figure 36 strong modifications produced by the spatial inhomogeneities in both the angular and low-energy structures can be appreciated (see Ciappina et al (2013a) for more details).

However in the case of low intensity regime (i.e. multiphoton regime, $\gamma \gg 1$ ) the scenario changes radically. In order to study this regime we use a laser electric field with $E_{0}=0.05$ a.u. of peak amplitude $\left(I=8.775 \times 10^{13} \mathrm{~W} \mathrm{~cm}^{-2}\right), \omega=0.25$ a.u. $(\lambda=182.5 \mathrm{~nm})$ and 6 complete optical cycles. The resulting Keldysh parameter $\gamma=5$ indicates the predominance of a multiphoton process (Arbó et al 2008). In figure 37 we show the two-dimensional electron distributions for the two cases discussed above. For the homogeneous case our calculation is identical to the one presented in Arbó et al (2008). We also notice the two panels present indistinguishable shape and magnitude. Hence the differences introduced by the spatial inhomogeneity are practically imperceptible in the multiphoton ionization regime.

\subsection{SFA and quantum orbits}

As for the case of HHG driven by spatially inhomogeneous fields, ATI can also be modeled by using the SFA. In order to do so, it is necessary to modify the SFA ingredients, namely the classical action and the saddle point equations. The latter are more complex, but appear to be solvable for the case of spatially linear inhomogeneous fields (for details see Shaaran et al (2013b)). Within SFA it is possible to investigate how the individual pairs of quantum orbits contribute to the photoelectron 

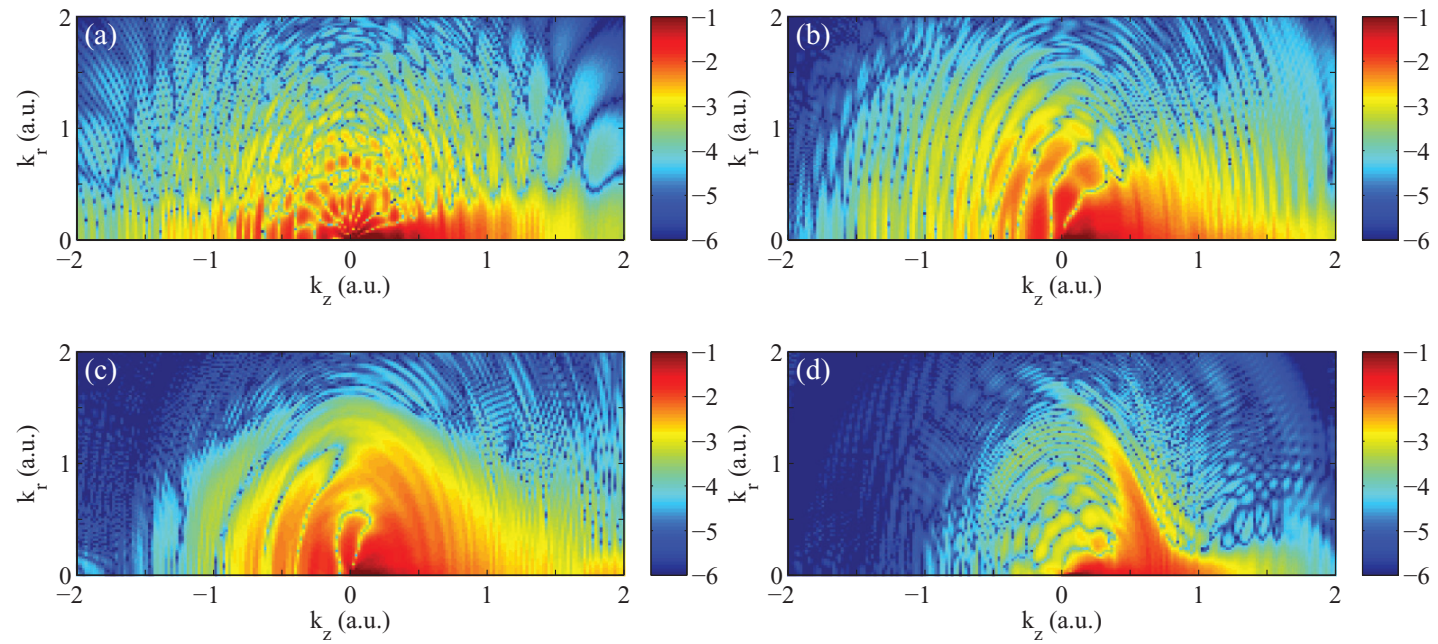

Figure 36. Two-dimensional electron momentum distributions (logarithmic scale) in cylindrical coordinates $\left(k_{z}, k_{r}\right)$ using the exact 3D-TDSE calculation for an hydrogen atom. The laser parameters are $I=5.0544 \times 10^{14} \mathrm{~W} \mathrm{~cm}^{-2}\left(E_{0}=0.12\right.$ a.u. $)$ and $\lambda=800 \mathrm{~nm}$. We have used a sin-squared shaped pulse with a total duration of four optical cycles ( $10 \mathrm{fs}$ ) with $\phi_{\mathrm{CEP}}=\pi / 2$. (a) $\varepsilon=0$ (homogeneous case), (b) $\varepsilon=0.002$, (c) $\varepsilon=0.003$ and (d) $\varepsilon=0.005$. Figure reused with permission from Ciappina et al (2013a). Copyright 2013 by American Physical Society.
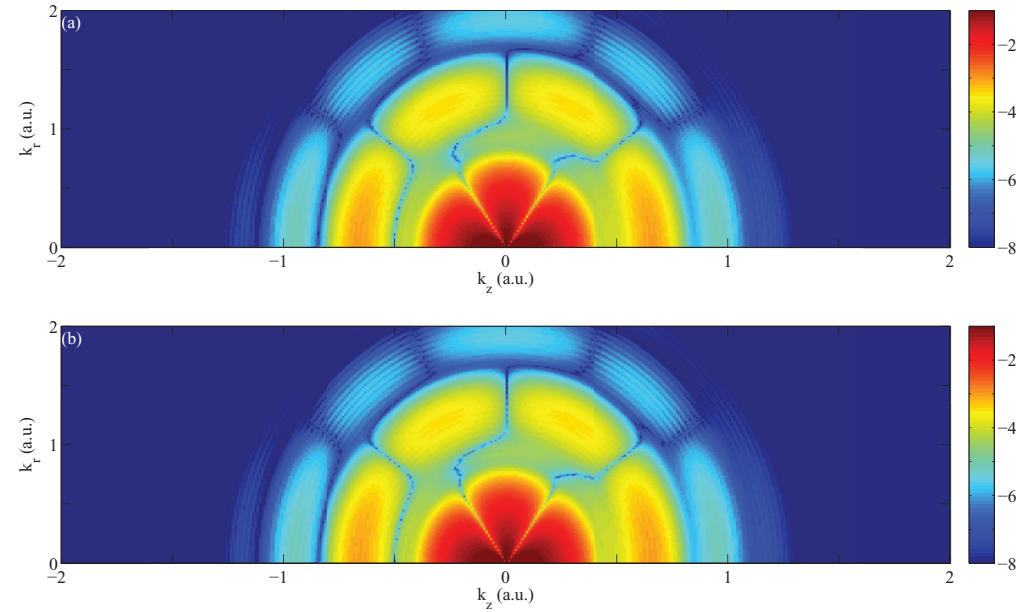

Figure 37. Two-dimensional electron momentum distributions (logarithmic scale) in cylindrical coordinates $\left(k_{z}, k_{r}\right)$ using the exact 3D-TDSE calculation for an hydrogen atom. The laser parameters are $E_{0}=0.05$ a.u. $\left(I=8.775 \times 10^{13} \mathrm{~W} \mathrm{~cm}^{-2}\right), \omega=0.25$ a.u. $(\lambda=182.5$ $\mathrm{nm}$ ) and $\phi_{\text {CEP }}=\pi / 2$. We employ a laser pulse with 6 total cycles. Panel (a) corresponds to the homogeneous case $(\varepsilon=0)$ and panel (b) is for $\varepsilon=0.005$. Figure reused with permission from Ciappina et al (2013a). Copyright 2013 by American Physical Society.

spectra and the two-dimensional electron momentum distributions. We demonstrate that the quantum orbits have a very different behavior in the spatially inhomogeneous field when compared to the homogeneous field. In the case of inhomogeneous fields, the ionization and rescattering times differ between neighboring cycles, despite the field being nearly monochromatic. Indeed, the contributions from one cycle may lead to a lower cutoff, while another may develop a higher cutoff. As was shown both by our quantum mechanical and classical models, our SFA model confirms that the ATI cutoff extends far beyond the semiclassical cutoff, as a function of inhomogeneity strength. In addition, the angular momentum distributions have very different features compared to the homogeneous case. For the neighboring cycles, the electron momentum distributions do not share the same absolute momentum, and as a consequence they do not have the same yield.

\subsection{Near-fields}

In this section we put forward the plausibility to perform ATI experiments by combining plasmonic enhanced nearfields and noble gases. The proposed experiment would take advantage of the plasmonic enhanced near-fields (also known as evanescent fields), which present a strong spatial nonhomogeneous character and the flexibility to use any atom or molecule in gas phase. A similar scheme was presented in section 8.5 , but now we are interested in generating highly energetic electrons, instead of coherent electromagnetic radiation. We employ the 1D-TDSE (see section 7.2) by including the actual functional form of metal nanoparticles plasmonic near-fields obtained from attosecond streaking measurements. We have chosen this particular nanostructure since its actual enhanced-field is known experimentally, while for the other nanostructures, like bow-ties (Kim et al 

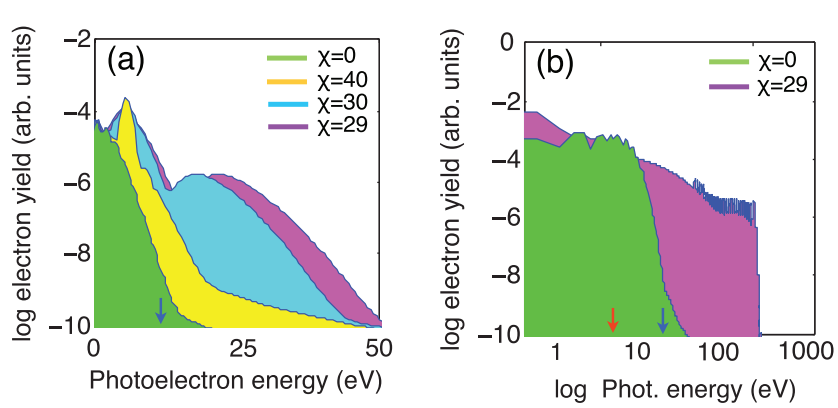

Figure 38. Energy resolved photoelectron spectra for Xe atoms driven by an electric enhanced near-field. In panel (a) the laser intensity after interacting with the metal nanoparticles is $I=2 \times 10^{13} \mathrm{~W} \mathrm{~cm}^{-2}$. We employ $\phi_{\mathrm{CEP}}=\pi / 2$ (cos-like pulses) and the laser wavelength and number of cycles remain unchanged with respect to the input pulse, i.e. $\lambda=720 \mathrm{~nm}$ and $n_{p}=5$ (13 fs in total). Panel (b) shows the output laser intensity of $I=5 \times 10^{13}$ $\mathrm{W} \mathrm{cm} \mathrm{cm}^{-2}$ (everything else is the same as in panel (a)). The arrow indicates two conventional classical limits: $2 U_{p}$ (in red) at $5.24 \mathrm{eV}$ and $10 U_{p}$ (in blue) at $26.2 \mathrm{eV}$, respectively. Figure reused with permission from Ciappina et al (2013b) and modified. Copyright 2013 by IOP Publishing.

2008), the actual plasmonic field is unknown. For most of the plasmonic nanostructures the enhanced field is theoretically calculated using the finite element simulation, which is based on an ideal system that may deviate significantly from actual experimental conditions. For instance, Kim et al (2008) states an intensity enhancement of 4 orders of magnitude (calculated theoretically) but the maximum harmonic measured was the 17 th, which corresponds to an intensity enhancement of only 2 orders of magnitude (for more details see Ciappina et al (2012a), Shaaran et al (2012a)). On the other hand, our numerical tools allow a treatment of a very general set of spatial nonhomogeneous fields such as those present in the vicinity of metal nanostructures (Kim et al 2008), dielectric nanoparticles (Zherebtsov et al 2011), or metal nanotips (Herink et al 2012). The kinetic energy for the electrons both direct and rescattered can be classically calculated and compared to quantum mechanical predictions (for more details see e.g. Ciappina et al (2013b)).

We have employed the same parameters as the ones used in section 8.5 , but now our aim is to compute the $P(E)$. In figure 38 we present the $P(E)$ calculated using 1D-TDSE for $\mathrm{Xe}$ atoms and for two different laser intensities, namely $I=2 \times 10^{13} \mathrm{~W} \mathrm{~cm}^{-2}$ (figure 38(a)) and $I=5 \times 10^{13} \mathrm{~W} \mathrm{~cm}^{-2}$ (figure 38(b)). In figure 38(a) each curve presents different values of $\chi$ : homogeneous case $(\chi \rightarrow \infty), \chi=40, \chi=35$ and $\chi=29$. For the homogeneous case there is a visible cutoff at $\approx 10.5 \mathrm{eV}$ confirming the well known ATI cutoff at $10 U_{p}$, which corresponds to those electrons that once ionized return to the core and elastically rescatter. On the other hand, for this particular intensity, the cutoff at $2 U_{p}(\approx 2.1 \mathrm{eV})$ developed by the direct ionized electrons is not visible in the spectrum.

For the spatial nonhomogeneous cases the cutoff of the rescattered electron is far beyond the $10 U_{p}$ classical limit, depending on the $\chi$ parameter chosen. As it is depicted in figure 38(a) the cutoff is extended as we decrease the value of $\chi$. For $\chi=40$ the cutoff is at around $14 \mathrm{eV}$, while for $\chi=29$ it is around $30 \mathrm{eV}$. The low energy region of the photoelectron spectra is sensitive to the atomic potential of the target and one would need to use the 3D-TDSE in order to model this region adequately. In this paper we are interested in the high energy region of the $P(E)$, which is very convenient because it is not greatly affected by the considered active media. Thus by employing 1D-TDSE the conclusions that can be taken from these highly energetic electrons are very reliable.

Figure 38(b) shows the $P(E)$ for the homogeneous case and for $\chi=29$ using a larger laser field intensity of $I=5 \times 10^{13}$ $\mathrm{W} \mathrm{cm}{ }^{-2}$, while keeping all other laser parameters fixed. From this plot we observe that the nonhomogeneous character of the laser enhanced electric field introduces a highly nonlinear behavior. For this intensity with $\chi=29$ it is possible to obtain very energetic electrons reaching values of several hundreds of $\mathrm{eV}$. This is a good indication that the nonlinear behavior of the combined system of the metallic nanoparticles and noble gas atoms could pave the way to generate $\mathrm{keV}$ electrons with tabletop laser sources. All the above quantum mechanical predictions can be directly confirmed by using classical simulations in the same way as for the case HHG (see section 8.5).

Here we propose generation of high energy photoelectrons using plasmonic-enhanced near-fields by combining metallic nanoparticles and noble gas atoms. Plasmonic-enhanced near-fields present a strong spatial dependence at a nanometer scale and this behavior introduces substantial changes in the laser-matter processes. We have modified the 1D-TDSE to model the ATI phenomenon in noble gases driven by the enhanced near-fields of such nanostructure. We predict a substantial extension in the cutoff position of the energy-resolved photoelectron spectra, far beyond the conventional $10 U_{p}$ classical limit. These new features are well reproduced by classical simulations. Our predictions would pave the way to the production of high energy photoelectrons reaching the $\mathrm{keV}$ regime by using a combination of metal nanoparticles and noble gases. In this kind of system each metal nanoparticle configures a laser nanosource with particular characteristics that allow not only the amplification of the input laser field, but also the modification of the laser-matter phenomena due to the strong spatial dependence of the generated coherent electromagnetic radiation.

\section{Other processes}

Most of the approaches applied to theoretically model $\mathrm{HHG}$ and ATI, both driven by spatial homogeneous and nonhomogeneous fields, are based on the hypothesis that the SAE approximation is good enough to describe both the harmonic emission and the laser ionized electron spectrum. For multielectronic processes, such as NSDI, clearly the SAE is insufficient. Studies of HHG considering two- and multi-electron effects have been performed by several authors Bandrauk and Lu (2005), Grobe and Eberly (1993), Koval et al (2007), Lappas et al (1996), Santra and Gordon (2006), Shi-Lin and Ting-Yun (2013). From these contributions, we conclude that depending on the atomic target properties and the laser frequency-intensity regime, multielectronic effects could play an 
important role in HHG (Grobe and Eberly 1993, Lappas et al 1996, Koval et al 2007). We should mention, however, that all these theoretical approaches have been developed for spatially homogeneous fields and that, to the best of our knowledge, studies of strong field phenomena driven by spatially inhomogeneous fields beyond the SAE have not been reported yet.

We have started to investigate how plasmonic fields modify the electron dynamics in a prototypical two-electron systems, namely the $\mathrm{He}$ atom and the negative hydrogen ion $\left(\mathrm{H}^{-}\right)$. To this end we employ both a 1D-TDSE and a reduced $1 \mathrm{D} \times 1 \mathrm{D}-\mathrm{TDSE}$ to model both systems in the SAE and the two-active electron (TAE) approximations, respectively. From these models, we trace out the analogies and differences in the HHG process from these two atomic systems, a priori very similar in their intrinsic structure (Chacón et al 2016).

\section{Conclusions, outlook and perspectives}

In this report on progress we have extensively reviewed, from both an experimental and theoretical viewpoints, the recent developments of the atto-nanophysics.

Nowadays, for the first time in the history of AMO physics we have at our disposal laser sources, which, combined with nanostructures, generate fields that exhibit spatial variation at a nanometric scale. This is the natural scale of the electron dynamics in atoms, molecules and bulk matter. Consequently, noticeable and profound changes occur in systems interacting with such spatially inhomogeneous fields. Using well known numerical techniques, based on solutions of Maxwell equations, one is able to model both the time and the spatial properties of these laser induced plasmonic fields. This is the first important step for the subsequent theoretical modelling of the strong field physical processes driven by them.

From a theoretical perspective, in the recent years there has been a comprehensive and continuous activity in atto-nanophysics. Indeed, all of the theoretical tools developed to tackle strong field processes driven by spatial homogeneous fields have been generalized and adapted to this new stage. Several open problems, however, still remain. For instance, the behaviour of complex systems, e.g. multielectronic atoms and molecules, under the influence of spatial inhomogeneous fields is an unexplored area-only few attempts to tackle this problem has been recently reported (Yavuz et al 2015, Chacón et al 2016, Yavuz et al 2016). In addition, and just to name another example, it was recently demonstrated that Rydberg atoms could be a plausible alternative as a driven media (Tikman et al 2016).

Several paths could be explored in the future. The manipulation and control of the plasmonic-enhanced fields appears as one them. From an experimental perspective this presents a tremendous challenge, considering the nanometric dimensions of the systems, although several experiments are planned in this direction, for instance combining metal nanotips and molecules in a gas phase. The possibility to tailor the electron trajectories at their natural scale is another avenue to be considered. By employing quantum control tools it would be possible, in principle theoretically, to drive the electron following a certain desired 'target', e.g. a one which results with the largest possible velocity, now with a time and spatial dependent driving field. The spatial shape of this field could be, subsequently, obtained by engineering a nanostructure.

The quest for HHG from plasmonic nano-structures, joint with an explosive amount of theoretical work, started with the controversial report of a Korean group on HHG from bow-tie metal nanostructures (Kim et al 2008). Let us mention at the end of this report a very recent result of the same group, which clearly seems to be well justified and, as such, opens new perspectives and ways toward efficient HHG in nano-structures. In this recent contribution the authors demonstrate $\mathrm{HHG}$ driven by plasmonic-enhanced fields experimentally by devising a metal-sapphire nanostructure that provides a solid tip as the HHG emitter instead of gaseous atoms. The fabricated solid tips are made of monocrystalline sapphire surrounded by a gold thin-film layer, and intended to produce coherent extreme ultraviolet (EUV) harmonics by the inter- and intraband oscillations of electrons driven by the incident laser. The metal-sapphire nanostructure enhances the incident laser field by means of SPPs triggering HHG directly from moderate femtosecond pulses of $0.1 \mathrm{TW} \mathrm{cm}^{-2}$ intensities. Measured EUV spectra show odd-order harmonics up to $60 \mathrm{~nm}$ wavelengths without the plasma atomic lines typically seen when using gaseous atoms as the HHG emitter. This experimental outcome confirms that the plasmonic HHG approach is a promising way to realize coherent EUV sources for nanoscale near-field applications in spectroscopy, microscopy, lithography, and attosecond physics (Han et al 2016). A new era of ultrafast physics is beginning!

\section{Acknowledgments}

This work was supported by the project ELI-Extreme Light Infrastructure-phase 2 (CZ.02.1.01/0.0/0.0/15_008/0000162) from European Regional Development Fund and in part by Max Planck POSTECH/KOREA Research Initiative Program [Grant No. 2011-0031558] through the National Research Foundation of Korea (NRF) funded by Ministry of Science, ICT \& Future Planning. ASL acknowledges Max Planck Center for Attosecond Science. MK acknowledges financial support by the Minerva Foundation and the Koshland Foundation. JAP-H and LR acknowledge to the Spanish Ministerio de Economía y Competitividad (FURIAM Project No. FIS2013-47741-R and PALMA project FIS2016-81056-R), Laserlab-Europe (EU-H2020 654148) and Junta de Castilla y León project CLP087U16. AB is funded by the Imperial College London Junior Research Fellowship. AZ acknowledges support from UK-EPSRC project EP/J002348/1 'CADAM'. ML and AC acknowledge the support of Spanish MINECO (Nacional Plan grants FOQUS FIS2013-46768-P and FISICATEAMO FIS2016-79508-P, SEVERO OCHOA grant/SEV-20150522), the National Science Centre, Poland-Synfonia grant 2016/20/W/ST4/00314, Fundació Cellex, Generalitat de Catalunya (AGAUR SGR 874 2014-2016 and CERCA/ Program), ERC AdG OSYRIS and EU FETPRO QUIC. $\mathrm{PH}$ acknowledges funding via the ERC Consolidator Grant 
NearFieldAtto, DFG-SPP 1840, DFG-SFB 953 and the Gordon and Betty Moore Foundation. MFC acknowledges Dennis Luck for helping with the artwork. BF, JS, WO and MFK acknowledge funding by the EU via the ERC Grant ATTOCO (no. 307203), the DFG via SPP 1840 and the excellence cluster 'Munich Centre for Advanced Photonics'. BF and MFK acknowledge support from Marco Allione and Enzo Di Fabrizio via the King Abdullah University of Science and Technology (KAUST).

\section{References}

Agostini P, Fabre F, Mainfray G, Petite G and Rahman N K 1979 Free-free transitions following six-photon ionization of xenon atoms Phys. Rev. Lett. 421127

Ahmad I et al 2009 Frontend light source for short-pulse pumped opcpa system Appl. Phys. B 97 529-36

Akturk S, D'Amico C and Mysyrowicz A 2008 Measuring ultrashort pulses in the single-cycle regime using frequencyresolved optical gating J. Opt. Soc. Am. B 25 A63-9

Altucci C, Nebbioso A, Benedetti R, Esposito R, Carafa V, Conte M, Micciarelli M, Altucci L and Velotta R 2012 Nonlinear protein-nucleic acid crosslinking induced by femtosecond uv laser pulses in living cells Laser Phys. Lett. 9234

Altucci C, Tisch J W G and Velotta R 2011 Single attosecond light pulses from multi-cycle laser sources J. of Mod. Opt. 58 1585-610

Ammosov M V, Delone N B and Kraĭnov V P 1986 Tunnel ionization of complex atoms and of atomic ions in an alternating electromagnetic field Sov. Phys._JETP 641191

Anderson A, Deryckx K S, Xu X G, Steinmeyer G and Raschke M B 2010 Few-femtosecond plasmon dephasing of a single metallic nanostructure from optical response function reconstruction by interferometric frequency resolved optical gating Nano Lett. 10 2519-24

Anker J N, Hall W P, Lyandres O, Shah N C, Zhao J and Van Duyne R P 2008 Biosensing with plasmonic nanosensors Nat. Mater. $7442-53$

Antoine P, L'Huillier A and Lewenstein M 1996 Attosecond pulse trains using high-order harmonics Phys. Rev. Lett. 771234

Apolonski A et al 2004 Observation of light-phase-sensitive photoemission from a metal Phys. Rev. Lett. 92073902

Apolonski A, Poppe A, Tempea G, Spielmann C, Udem T, Holzwarth R, Hänsch T W and Krausz F 2000 Controlling the phase evolution of few-cycle light pulses Phys. Rev. Lett. $85740-3$

Arbó D G, Miraglia J E, Gravielle M S, Schiessl K, Persson E and Burgdörfer J 2008 Coulomb-volkov approximation for near-threshold ionization by short laser pulses Phys. Rev. A 77013401

Arnold A et al 2013 Comparison of scalable fast methods for longrange interactions Phys. Rev. E $\mathbf{8 8} 063308$

Bagheri S, Weber K, Gissibl T, Weiss T, Neubrech F and Giessen H 2015 Fabrication of square-centimeter plasmonic nanoantenna arrays by femtosecond direct laser writing lithography: effects of collective excitations on SEIRA enhancement ACS Photonics $2779-86$

Bainbridge A R and Bryan W A 2014 Velocity map imaging of femtosecond laser induced photoelectron emission from metal nanotips New J. Phys. 16103031

Balciunas T, Fourcade-Dutin C, Fan G, Witting T, Voronin A A, Zheltikov A M, Gerome F, Paulus G G, Baltuska A and Benabid F 2015 A strong-field driver in the single-cycle regime based on selfcompression in a kagome fibre Nat. Commun. 66117
Baltuska A et al 2003a Attosecond control of electronic processes by intense light fields Nature $\mathbf{4 2 1} 611-5$

Baltuska A, Uiberacker M, Goulielmakis E, Kienberger R, Yakovlev V S, Udem T, Hansch T W and Krausz F 2003b Phase-controlled amplification of few-cycle laser pulses IEEE J. Sel. Top. Quantum Electron. 9 972-89

Bandrauk A D and Lu H-Z 2005 Harmonic generation in a 1d model of $\mathrm{H}_{2}$ with single and double ionization J. Phys. B: At. Mol. Opt. Phys. 382529

Barwick B, Corder C, Strohaber J, Chandler-Smith N, Uiterwaal C and Batelaan H 2007 Laser-induced ultrafast electron emission from a field emission tip New J. Phys. 9142

Barwick B, Flannigan D J and Zewail A H 2009 Photon-induced near-field electron microscopy Nature 462 902-6

Batani D, Joachain C J, Martellucci S and Chester A N 2001 Atoms, Solids, and Plasmas in Super-Intense Laser Fields (New York: Academic)

Becker W, Grasbon F, Kopold R, Milošević D B, Paulus G G and Walther H 2002 Above-threshold ionization: from classical features to quantum effects Advances In Atomic, Molecular, and Optical Physics ed B Bederson and H Walter (San Diego, CA: Academic) p 35

Belshaw L, Calegari F, Duffy M J, Trabattoni A, Poletto L, Nisoli M and Greenwood J B 2012 Observation of ultrafast charge migration in an amino acid J. Phys. Chem. Lett. 3 3751-4

Bergues B, Kübel M, Kling N G, Burger C and Kling M F 2015 Single-cycle non-sequential double ionization IEEE J. Sel. Top. Quantum Electron. 218701009

Berweger S, Atkin J M, Olmon R L and Raschke M B 2012 Light on the tip of a needle: plasmonic nanofocusing for spectroscopy on the nanoscale J. Phys. Chem. Lett. 3 945-52

Bionta M R, Chalopin B, Champeaux J P, Faure S, Masseboeuf A, Moretto-Capelle P and Chatel B 2014 Laser-induced electron emission from a tungsten nanotip: identifying above threshold photoemission using energy-resolved laser power dependencies J. Mod. Opt. 61 833-8

Bionta M R, Chalopin B, Masseboeuf A and Chatel B 2015 First results on laser-induced field emission from a cnt-based nanotip Ultramicroscopy 159 152-5 (1st Int. Conf. on Atom Probe Tomography and Microscopy)

Blaga C I, Xu J, DiChiara A D, Sistrunk E, Zhang K, Agostini P, Miller T A, DiMauro L F and Lin C D 2012 Imaging ultrafast molecular dynamics with laser-induced electron diffraction Nature 483 194-7

Bohren C F and Huffman D R 1998 Absorption and Scattering of Light by Small Particles (New York: Wiley)

Bolten M, Fahrenberger F, Halver R, Heber F, Hofmann M, Kabadshow I, Lenz O, Pippig M and Sutmann G 2016 ScaFaCoS, C subroutine library (http://scafacos.github.com)

Borisov A G, Echenique P M and Kazansky A K 2012 Attostreaking with metallic nano-objects New. J. Phys. 14023036

Borisov A G, Sánchez-Portal D, Kazansky A K and Echenique P M 2013 Resonant and nonresonant processes in attosecond streaking from metals Phys. Rev. B 87121110

Bormann R, Gulde M, Weismann A, Yalunin S V and Ropers C 2010 Tip-enhanced strong-field photoemission Phys. Rev. Lett. 105147601

Bormann R, Strauch S, Schäfer S and Ropers C 2015 An ultrafast electron microscope gun driven by two-photon photoemission from a nanotip cathode J. Appl. Phys. 118173105

Bothschafter E M, Schiffrin A, Yakovlev V S, Azzeer A M, Krausz F, Ernstorfer R and Kienberger R 2010 Collinear generation of ultrashort uv and xuv pulses Opt. Express 18 9173-80

Bouhelier A, Beversluis M, Hartschuh A and Novotny L 2003 Near-field second-harmonic generation induced by local field enhancement Phys. Rev. Lett. 90013903 
Brabec T and Krausz F 2000 Intense few-cycle laser fields: frontiers of nonlinear optics Rev. Mod. Phys. 72 545-91

Brizuela F, Heyl C M, Rudawski P, Kroon D, Rading L, Dahlström J M, Mauritsson J, Johnsson P, Arnold C L and L'Huillier A 2013 Efficient high-order harmonic generation boosted by below-threshold harmonics Sci. Rep. 31410

Bunkin F V and Fedorov M V 1965 Cold emission of electrons from the surface of a metal in a strong radiation field Sov. Phys.-JETP 21 896-9

Calegari F et al 2014 Ultrafast electron dynamics in phenylalanine initiated by attosecond pulses Science 346336

Calegari F, Sansone G, Stagira S, Vozzi C and Nisoli M 2016a Advances in attosecond science J. Phys. B: At. Mol. Opt. Phys. 49062001

Calegari F, Trabattoni A, Palacios A, Ayuso D, Castrovilli M C, Greenwood J B, Decleva P, Martín F and Nisoli M 2016b Charge migration induced by attosecond pulses in bio-relevant molecules J. Phys. B: At. Mol. Opt. Phys. 49142001

del Campo A and Arzt E 2008 Fabrication approaches for generating complex micro- and nanopatterns on polymeric surfaces Chem. Rev. 108 911-45

Cao X, Jiang S, Yu C, Wang Y, Bai L and Lu R 2014 Generation of isolated sub-10-attosecond pulses in spatially inhomogenous two-color fields Opt. Express 22 26153-61

Caprez A, Barwick B and Batelaan H 2007 Macroscopic test of the Aharonov-Bohm effect Phys. Rev. Lett. 99210401

Cavalieri A L et al 2007a Intense 1.5-cycle near infrared laser waveforms and their use for the generation of ultra-broadband soft-x-ray harmonic continua New J. Phys. 9242

Cavalieri A L et al 2007b Attosecond spectroscopy in condensed matter Nature 449 1029-32

Chacón A, Ciappina M F and Lewenstein M 2015 Numerical studies of light-matter interaction driven by plasmonic fields: the velocity gauge Phys. Rev. A 92063834

Chacón A, Ciappina M F and Lewenstein M 2016 Double-electron recombination in high-order-harmonic generation driven by spatially inhomogeneous fields Phys. Rev. A 94043407

Chaturvedi P, Hsu K H, Kumar A, Fung K H, Mabon J C and Fang N X 2009 Imaging of plasmonic modes of silver nanoparticles using high-resolution cathodoluminescence spectroscopy ACS Nano 3 2965-74

Chew S H et al 2012 Time-of-flight-photoelectron emission microscopy on plasmonic structures using attosecond extreme ultraviolet pulses Appl. Phys. Lett. 100051904

Choi S, Ciappina M F, Pérez-Hernández J A, Landsman A S, Kim Y-J, Kim S C and Kim D E 2016 Active tailoring of nanoantenna plasmonic field using few-cycle laser pulses Phys. Rev. A 93021405

Chung J-H and Weiner A M 2001 Ambiguity of ultrashort pulse shapes retrieved from the intensity autocorrelation and the power spectrum IEEE J. Sel. Top. Quantum Electron. 7 656-66

Ciappina M F, Aćimović S S, Shaaran T, Biegert J, Quidant R and Lewenstein M 2012a Enhancement of high harmonic generation by confining electron motion in plasmonic nanostrutures Opt. Express 20 26261-74

Ciappina M F, Biegert J, Quidant R and Lewenstein M 2012b Highorder-harmonic generation from inhomogeneous fields Phys. Rev. A 85033828

Ciappina M F, Pérez-Hernández J A and Lewenstein M 2014a Classstrong: classical simulations of strong field processes Comput. Phys. Commun. 185 398-406

Ciappina M F, Pérez-Hernández J A, Roso L, Zaïr A and Lewenstein M 2015 High-order harmonic generation driven by plasmonic fields: a new route towards the generation of uv and xuv photons? J. Phys.: Conf. Ser. $\mathbf{6 0 1} 012001$

Ciappina M F, Pérez-Hernández J A, Shaaran T, Biegert J, Quidant R and Lewenstein M 2012 Above-threshold ionization by few-cycle spatially inhomogeneous fields Phys. Rev. A 86023413
Ciappina M F, Pérez-Hernández J A, Shaaran T and Lewenstein M 2014b Coherent xuv generation driven by sharp metal tips photoemission Eur. Phys. J. D 68172

Ciappina M F, Pérez-Hernández J A, Shaaran T, Lewenstein M, Krüger M and Hommelhoff P 2014c High-order harmonic generation driven by metal nanotip photoemission: theory and simulations Phys. Rev. A 89013409

Ciappina M F, Pérez-Hernández J A, Shaaran T, Roso L and Lewenstein M 2013a Electron-momentum distributions and photoelectron spectra of atoms driven by an intense spatially inhomogeneous field Phys. Rev. A 87063833

Ciappina M F, Shaaran T, Guichard R, Pérez-Hernández J A, Roso L, Arnold M, Siegel T, Zaïr A and Lewenstein M 2013b High energy photoelectron emission from gases using plasmonic enhanced near-fields Las. Phys. Lett. 10105302

Ciappina M F, Shaaran T and Lewenstein M 2013c High order harmonic generation in noble gases using plasmonic field enhancement Ann. Phys. 525 97-106

Cirac C, Hill R T, Mock J J, Urzhumov Y, FernándezDomínguez A I, Maier S A, Pendry J B, Chilkoti A and Smith D R 2012 Probing the ultimate limits of plasmonic enhancement Science 337 1072-4

Clark J N et al 2015 Imaging transient melting of a nanocrystal using an x-ray laser Proc. Natl Acad. Sci. USA 112 7444-8

Corkum P B 1993 Plasma perspective on strong field multiphoton ionization Phys. Rev. Lett. 711994

Corkum P B, Burnett N H and Ivanov M Yu 1994 Subfemtosecond pulses Opt. Lett. 19 1870-2

Corkum P B and Krausz F 2007 Attosecond science Nat. Phys. 3 381-7

Da B, Shinotsuka H, Yoshikawa H, Ding Z J and Tanuma S 2014 Extended mermin method for calculating the electron inelastic mean free path Phys. Rev. Lett. 113063201

Deubel M, von Freymann G, Wegener M, Pereira S, Busch K and Soukoulis C M 2004 Direct laser writing of three-dimensional photonic-crystal templates for telecommunications Nat. Mater 3 444-7

Dietrich P, Krausz F and Corkum P B 2000 Determining the absolute carrier phase of a few-cycle laser pulse Opt. Lett. 25 16-8

Ditmire T, Smith R A, Tisch J W G and Hutchinson M H R 1997a High intensity laser absorption by gases of atomic clusters Phys. Rev. Lett. 783121

Ditmire T, Tisch J W G, Springate E, Mason M B, Hay N, Smith R A, Marangos J and Hutchinson M H R 1997b Highenergy ions produced in explosions of superheated atomic clusters Nature 38654

Dombi P, Hörl A, Rácz P, Marton I, Trügler A, Krenn J R and Hohenester U 2013 Ultrafast strong-field photoemission from plasmonic nanoparticles Nano Lett. 13 674-8

Dombi P et al 2010 Observation of few-cycle, strong-field phenomena in surface plasmon fields Opt. Express 18 24206-12

Donnelly T D, Rust M, Weiner I, Allen M, Smith R A, Steinke C A, Wilks S, Zweiback J, Cowan T E and Ditmire T 2001 Hard $\mathrm{X}$-ray and hot electron production from intense laser irradiation of wavelength-scale particles J. Phys. B: At. Mol. Opt. Phys. $34 \mathrm{~L} 313$

Drescher M, Hentschel M, Kienberger R, Tempea G, Spielmann C, Reider G A, Corkum P B and Krausz F 2001 X-ray pulses approaching the attosecond frontier Science 291 1923-7

Drescher M, Hentschel M, Kienberger R, Uiberacker M, Yakovlev V, Scrinzi A, Westerwalbesloh Th, Kleineberg U, Heinzmann U and Krausz F 2002 Time-resolved atomic inner-shell spectroscopy Nature 419 803-7

Ebadi H 2014 Interferences induced by spatially nonhomogeneous fields in high-harmonic generation Phys. Rev. A 89053413

Ehberger D, Hammer J, Eisele M, Krüger M, Noe J, Högele A and Hommelhoff P 2015 Highly coherent electron beam from a laser-triggered tungsten needle tip Phys. Rev. Lett. 114227601 
Einstein A 1905 Über einen die erzeugung und verwandlung des lichtes betreffenden heuristischen gesichtspunkt Annalen der Physik 322 132-48

Esteban R, Borisov A G, Nordlander P and Aizpurua J 2012 Bridging quantum and classical plasmonics with a quantumcorrected model Nat. Commun. 3825

Fabris D, Witting T, Okell W A, Walke D J, Matia-Hernando P, Henkel J, Barillot T R, Lein M, Marangos J P and Tisch J W G 2015 Synchronized pulses generated at $20 \mathrm{eV}$ and $90 \mathrm{eV}$ for attosecond pump-probe experiments Nat. Photon. $9383-7$

Faisal F H M 1987 Theory of Multiphoton Processes (New York: Springer)

Feist A, Echternkamp K E, Schauss J, Yalunin S V, Schäfer S and Ropers C 2015 Quantum coherent optical phase modulation in an ultrafast transmission electron microscope Nature $521200-3$

Feng L and Liu H 2015 Attosecond extreme ultraviolet generation in cluster by using spatially inhomogeneous field Phys. Plasmas 22013107

Feng L, Yuan M and Chu T 2013 Attosecond x-ray source generation from two-color polarized gating plasmonic field enhancement Phys. Plasmas 20122307

Feng X, Gilbertson S, Mashiko H, Wang H, Khan S D, Chini M, Wu Y, Zhao K and Chang Z 2009 Generation of isolated attosecond pulses with 20-28 femtosecond lasers Phys. Rev. Lett. $\mathbf{1 0 3} 183901$

Fernández-García R, Sonnefraud Y, Fernández-Domínguez A I, Giannini V and Maier S A 2014 Design considerations for near-field enhancement in optical antennas Contemp. Phys. $551-11$

Fernandez-Varea J M, Mayol R, Liljequist D and Salvat F 1993 Inelastic scattering of electrons in solids from a generalized oscillator strength model using optical and photoelectric data J. Phys.: Condens. Matter 53593

Ferrari F, Calegari F, Lucchini M, Vozzi C, Stagira S, Sansone G and Nisoli M 2010 High-energy isolated attosecond pulses generated by above-saturation few-cycle fields Nat. Photon. 4 875-9

Ferré A et al 2015 A table-top ultrashort light source in the extreme ultraviolet for circular dichroism experiments Nat. Photon. 9 93-8

Fetić B, Kalajdžić K and Milošević D B 2012 High-order harmonic generation by a spatially inhomogeneous field Ann. Phys. 525 107-17

Fieß M, Schultze M, Goulielmakis E, Dennhardt B, Gagnon J, Hofstetter M, Kienberger R and Krausz F 2010 Versatile apparatus for attosecond metrology and spectroscopy Rev. Sci. Instrum. 81093103

Foreman S M, Kealhofer C, Skulason G E, Klopfer B B and Kasevich M A 2013 Ultrafast microfocus x-ray source based on a femtosecond laser-triggered tip Ann. Phys. 525 L19-22

Förg B et al 2016 Attosecond nanoscale near-field sampling Nat. Commun. 711717

Fowler R H and Nordheim L 1928 Electron emission in intense electric fields Proc. R. Soc. Lond. A 119 173-81

Frank F et al 2012 Invited review article: technology for attosecond science Rev. Sci. Instrum. 83071101

Frassetto F, Trabattoni A, Anumula S, Sansone G, Calegari F, Nisoli M and Poletto L 2014 High-throughput beamline for attosecond pulses based on toroidal mirrors with microfocusing capabilities Rev. Sci. Instrum. 85103115

Fröhlich H and Pelzer H 1955 Plasma oscillations and energy loss of charged particles in solids Proc. Phys. Soc. A 68525

Fursey G N 2005 Field Emission in Vacuum Microelectronics (New York: Academic)

Ganter R, Bakker R, Gough C, Leemann S C, Paraliev M, Pedrozzi M, Le Pimpec F, Schlott V, Rivkin L and Wrulich A 2008 Laser-photofield emission from needle cathodes for lowemittance electron beams Phys. Rev. Lett. 100064801
Ghimire S, DiChiara A D, Sistrunk E, Agostini P, DiMauro L F and Reis D A 2011 Observation of high-order harmonic generation in a bulk crystal Nat. Phys. 7 138-41

Gong Y, Joly A G, Hu D, El-Khoury P Z and Hess W P 2015 Ultrafast imaging of surface plasmons propagating on a gold surface Nano Lett. 15 3472-8

Goulielmakis E et al 2008 Single-cycle nonlinear optics Science 320 1614-7

Grobe R and Eberly J H 1993 One-dimensional model of a negative ion and its interaction with laser fields Phys. Rev. A 484664

Guggenmos A et al 2015 Chromium/scandium multilayer mirrors for isolated attosecond pulses at $145 \mathrm{eV}$ Opt. Lett. $402846-9$

Gulde M, Schweda S, Storeck G, Maiti M, Yu H K, Wodtke A M, Schäfer S and Ropers C 2014 Ultrafast low-energy electron diffraction in transmission resolves polymer/graphene superstructure dynamics Science 345 200-4

Gumbrell E T, Comley A J, Hutchinson M H R and Smith R A 2001 Intense laser interactions with sprays of submicron droplets Phys. Plasmas $\mathbf{8} 1329$

Habteyes T G, Dhuey S, Wood E, Gargas D, Cabrini S, Schuck P J, Alivisatos A P and Leone S R 2012 Metallic adhesion layer induced plasmon damping and molecular linker as a nondamping alternative ACS Nano 6 5702-9

Hafner C 1999 Post-modern Electromagnetics: Using Intelligent MaXwell Solvers (New York: Wiley)

Han S, Kim H, Kim Y W, Kim Y-J, Kim S, Park I-Y and Kim S-W 2016 High-harmonic generation by field enhanced femtosecond pulses in metal-sapphire nanostructure Nat. Commun. 713105

Hanke T, Krauss G, Träutlein D, Wild B, Bratschitsch R and Leitenstorfer A 2009 Efficient nonlinear light emission of single gold optical antennas driven by few-cycle near-infrared pulses Phys. Rev. Lett. 103257404

Hansen P M, Bhatia V K, Harrit N and Oddershede L 2005 Expanding the optical trapping range of gold nanoparticles Nano Lett. 5 1937-42

Hartschuh A 2008 Tip-enhanced near-field optical microscopy Angew. Chem. Int. Ed. 47 8178-91

Hassan M Th et al 2016 Optical attosecond pulses and tracking the nonlinear response of bound electrons Nature $\mathbf{5 3 0} 66-70$

He L, Wang Z, Li Y, Zhang Q, Lan P and Lu P 2013 Wavelength dependence of high-order-harmonic yield in inhomogeneous fields Phys. Rev. A $\mathbf{8 8} 053404$

Hemmers O, Whitfield S B, Glans P, Wang H, Lindle D W, Wehlitz R and Sellin I A 1998 High-resolution electron timeof-flight apparatus for the soft x-ray region Rev. Sci. Instrum. 69 3809-17

Henkel J, Witting T, Fabris D, Lein M, Knight P L, Tisch J W G and Marangos J P 2013 Prediction of attosecond light pulses in the vuv range in a high-order-harmonic-generation regime Phys. Rev. A 87043818

Hentschel M, Kienberger R, Spielmann C, Reider G A, Milosevic N, Brabec T, Corkum P B, Heinzmann U, Drescher M and Krausz F 2001 Attosecond metrology Nature 414 509-13

Herink G, Solli D R, Gulde M and Ropers C 2012 Field-driven photoemission from nanostructures quenches the quiver motion Nature 483 190-3

Herink G, Wimmer L and Ropers C 2014 Field emission at terahertz frequencies: AC-tunneling and ultrafast carrier dynamics New J. Phys. 16123005

van Linden van den Heuvell H B and Muller H G 1988 Multiphoton Processes ed S J Smith and P L Knight (Cambridge: Cambridge University Press) p 25

Higuchi T, Maisenbacher L, Liehl A, Dombi P and Hommelhoff P 2015 A nanoscale vacuum-tube diode triggered by few-cycle laser pulses Appl. Phys. Lett. 106051109

Hilbert S A, Neukirch A, Uiterwaal C J G J and Batelaan H 2009 Exploring temporal and rate limits of laser-induced electron emission J. Phys. B: At. Mol. Opt. Phys. 42141001 
Hobbs R G, Yang Y, Fallahi A, Keathley P D, De Leo E, Kärtner F X, Graves W S and Berggren K K 2014 High-yield, ultrafast, surface plasmon-enhanced, Au nanorod optical field electron emitter arrays ACS Nano 8 11474-82

Hoffrogge J, Stein J P, Krüger M, Förster M, Hammer J, Ehberger D, Baum P and Hommelhoff P 2014 Tip-based source of femtosecond electron pulses at $30 \mathrm{keV} \mathrm{J.} \mathrm{Appl.} \mathrm{Phys.}$ 115094506

Hommelhoff P, Kealhofer C and Kasevich M A 2006a Femtosecond laser meets field emission tip-a sensor for the carrier envelope phase? Proc. the 2006 IEEE Int. Frequency Control Symp. and Expositions vol 1 and 2 pp 470-4

Hommelhoff P, Kealhofer C and Kasevich M A 2006b Ultrafast electron pulses from a tungsten tip triggered by low-power femtosecond laser pulses Phys. Rev. Lett. 97247402

Hommelhoff P and Kling M F 2015 Attosecond Nanophysics: from Basic Science to Applications (Berlin: Wiley)

Hommelhoff P, Sortais Y, Aghajani-Talesh A and Kasevich M A 2006c Field emission tip as a nanometer source of free electron femtosecond pulses Phys. Rev. Lett. 96077401

Huppert M, Jordan I and Wörner H J 2015 Attosecond beamline with actively stabilized and spatially separated beam paths Rev. Sci. Instrum. 86123106

Husakou A and Herrmann J 2014 Quasi-phase-matched highharmonic generation in composites of metal nanoparticles and a noble gas Phys. Rev. A 90023831

Husakou A, Im S-J and Herrmann J 2011a Theory of plasmonenhanced high-order harmonic generation in the vicinity of metal nanostructures in noble gases Phys. Rev. A 83043839

Husakou A, Im S-J, Kim K H and Herrmann J 2015 High harmonic generation assisted by metal nanostructures and nanoparticles Progress in Nonlinear Nano-Optics ed S Sakabe et al (Berlin: Springer) pp 251-68

Husakou A, Kelkensberg F, Herrmann J and Vrakking M J J 2011b Polarization gating and circularly-polarized high harmonic generation using plasmonic enhancement in metal nanostructures Opt. Express 19 25346-54

Huth F, Chuvilin A, Schnell M, Amenabar I, Krutokhvostov R, Lopatin S and Hillenbrand R 2013 Resonant antenna probes for tip-enhanced infrared near-field microscopy Nano Lett. 13 1065-72

Iaconis C and Walmsley I A 1998 Spectral phase interferometry for direct electric-field reconstruction of ultrashort optical pulses Opt. Lett. 23 792-4

Inouye Y and Kawata S 1994 Near-field scanning optical microscope with a metallic probe tip Opt. Lett. 19 159-61

Itatani J, Quéré F, Yudin G L, Ivanov M Yu, Krausz F and Corkum P B 2002 Attosecond streak camera Phys. Rev. Lett. 88173903

Itri F et al 2016 Femtosecond uv-laser pulses to unveil proteinprotein interactions in living cells Cell. Mol. Life Sci. 73637

Jackson J D 1999 Classical Electrodynamics (New York: Wiley)

Jain P K, Eustis S and El-Sayed M A 2006 Plasmon coupling in nanorod assemblies: Optical absorption, discrete dipole approximation simulation, and exciton-coupling model J. Phys. Chem. B 110 18243-53

Joachain C J, Kylstra N J and Potvliege R M 2003 Atoms in intense, ultrashort laser pulses: non-dipole and relativistic effects J. Mod. Opt. $\mathbf{5 0} 313$

Joachain C J, Kylstra N J and Potvliege R M 2012 Atoms in Intense Laser Fields (Cambridge: Cambridge University Press)

Jones D J, Diddams S A, Ranka J K, Stentz A, Windeler R S, Hall J L and Cundiff S T 2000 Carrier-envelope phase control of femtosecond mode-locked lasers and direct optical frequency synthesis Science $\mathbf{2 8 8}$ 635-9

Juffmann T, Klopfer B B, Skulason G E, Kealhofer C, Xiao F, Foreman S M and Kasevich M A 2015 Ultrafast time-resolved photoelectric emission Phys. Rev. Lett. 115264803

Kameta K, Kouchi N, Ukai M and Hatano Y 2002 Photoabsorption, photoionization, and neutral-dissociation cross sections of simple hydrocarbons in the vacuum ultraviolet range J. Electron Spectrosc. Relat. Phenom. 123 225-38

Kane D J 1999 Recent progress toward real-time measurement of ultrashort laser pulses IEEE J. Quantum Electron. 35 421-31

Kane D J, Rodríguez G, Taylor A J and Clement T S 1997 Simultaneous measurement of two ultrashort laser pulses from a single spectrogram in a single shot J. Opt. Soc. Am. B 14 935-43

Kane D J and Trebino R 1993 Characterization of arbitrary femtosecond pulses using frequency-resolved optical gating IEEE J. Quantum Electron. 29 571-9

Kauranen M and Zayats A V 2012 Nonlinear plasmonics Nat. Photon. $9737-48$

Kazansky A K and Echenique P M 2009 One-electron model for the electronic response of metal surfaces to subfemtosecond photoexcitation Phys. Rev. Lett. 102177401

Kealhofer C, Foreman S M, Gerlich S and Kasevich M A 2012 Ultrafast laser-triggered emission from hafnium carbide tips Phys. Rev. B 86035405

Keathley P D, Sell A, Putnam W P, Guerrera S, Velasquez-García L and Kärtner F X 2013 Strong-field photoemission from silicon field emitter arrays Ann. Phys. 525 144-50

Keldysh L V 1965 Ionization in the field of a strong electromagnetic wave Sov. Phys.-JETP 201307

Kelkensberg F, Koenderink A F and Vrakking M J J 2012 Attosecond streaking in a nano-plasmonic field New J. Phys. 14093034

Kienberger R et al 2004 Atomic transient recorder Nature 427 817-21

Kim K T, Zhang C, Shiner A D, Schmidt B E, Légaré F, Villeneuve D M and Corkum P B 2013 Petahertz optical oscilloscope Nat. Photon. 7 958-62

Kim S, Jin J, Kim Y-J, Park I-Y, Kim Y and Kim S-W 2008 Highharmonic generation by resonant plasmon field enhancement Nature $\mathbf{4 5 3} 757-60$

Kim S, Jin J, Kim Y-J, Park I-Y, Kim Y and Kim S-W 2012 Reply nature Nature 485 E1-3

Koval P, Wilken F, Bauer D and Keitel C H 2007 Nonsequential double recombination in intense laser fields Phys. Rev. Lett. 98043904

Krasovskii E E 2011 Attosecond spectroscopy of solids: streaking phase shift due to lattice scattering Phys. Rev. B 84195106

Krausz F 2016 The birth of attosecond physics and its coming of age Phys. Scr. 91063011

Krausz F and Ivanov M 2009 Attosecond physics Rev. Mod. Phys. 81 163-234

Krausz F and Stockman M I 2014 Attosecond metrology: from electron capture to future signal processing Nat. Photon. 8 205-13

Krüger M, Förster M and Hommelhoff P 2014 Self-probing of metal nanotips by rescattered electrons reveals the nano-optical near-field J. Phys. B: At. Mol. Opt. Phys. 47124022

Krüger M, Schenk M, Förster M and Hommelhoff P 2012a Attosecond physics in photoemission from a metal nanotip J. Phys. B: At. Mol. Opt. Phys. 45074006

Krüger M, Schenk M and Hommelhoff P 2011 Attosecond control of electrons emitted from a nanoscale metal tip Nature $47578-81$

Krüger M, Schenk M, Hommelhoff P, Wachter G, Lemell C and Burgdörfer J 2012b Interaction of ultrashort laser pulses with metal nanotips: a model system for strong-field phenomena New J. Phys. 14085019

Kübel M et al 2016 Steering proton migration in hydrocarbons using intense few-cycle laser fields Phys. Rev. Lett. 116193001

Kuchiev M Yu 1987 Atomic antenna JETP Lett. 45 404-6

Kulander K C, Schafer K J and Krause J L 1993 Dynamics of shortpulse excitation, ionization and harmonic conversion SuperIntense Laser-Atom Physics ed B Piraux et al (New York: Plenum) pp 95-110 
Kusa F, Echternkamp K E, Herink G, Ropers C and Ashihara S 2015 Optical field emission from resonant gold nanorods driven by femtosecond mid-infrared pulses $A I P A d v$. 5077138

Lafrate G J, Ziegler J F and Nass M J 1980 Application of lindhard's dielectric theory to the stopping of ions in solids J. Appl. Phys. 51 984-7

Landsman A S and Keller U 2015 Attosecond science and the tunnelling time problem Phys. Rep. 547 1-24

Lappas D G, Sanpera A, Watson J B, Burnett K, Knight P L, Grobe R and Eberly J H 1996 Two-electron effects in harmonic generation and ionization from a model he atom J. Phys. B: At Mol. Opt. Phys. 29 L619-27

Lemell C, Solleder B, Tökési K and Burgdörfer J 2009 Simulation of attosecond streaking of electrons emitted from a tungsten surface Phys. Rev. A 79062901

Leo G, Altucci C, Bourgoin-Voillard S, Gravagnuolo A M, Esposito R, Marino G, Costello C E, Velotta R and Birolo L 2013 Ultraviolet laser-induced cross-linking in peptides Rapid Commun. Mass Spectrom. 271660

Lewenstein M, Balcou P, Ivanov M Y, L'Huillier A and Corkum P B 1994 Theory of high-harmonic generation by lowfrequency laser fields Phys. Rev. A 492117

Lewenstein M and L'Huillier A 2009 Principles of single atom physics: high-order harmonic generation, above-threshold ionization and non-sequential ionization Strong Field Laser Physics ed T Brabec (New York: Springer) pp 147-83

L'Huiller A, Lewenstein M, Salières P, Balcou Ph, Ivanov M Yu, Larsson J and Wahlström C G 1993 High-order harmonicgeneration cutoff Phys. Rev. A 48 R3433

L'Huiller A, Lompre L A, Mainfray G and Manus C 1983 Multiply charged ions induced by multiphoton absorption in rate gases at $0.53 \mu \mathrm{m}$ Phys. Rev. A 272503

L'Huillier A, Schafer K J and Kulander K C 1991 Theoretical aspects of intense field harmonic generation J. Phys. B: At Mol. Opt. Phys. 243315

$\mathrm{Li} \mathrm{H}$ et al 2015 Coherent electronic wave packet motion in $\mathrm{C}_{60}$ controlled by the waveform and polarization of few-cycle laser fields Phys. Rev. Lett. 114123004

Liao Q and Thumm U 2014 Attosecond time-resolved photoelectron dispersion and photoemission time delays Phys. Rev. Lett. 112023602

Limpert J, Hädrich S, Rothhardt J, Krebs M, Eidam T, Schreiber T and Tünnermann A 2011 Ultrafast fiber lasers for strong-field physics experiments Laser Photon. Rev. 5 634-46

Lindhard J 1954 K. Dan. Vidensk. Selsk. Mat. Fys. Medd. 28 (no. 8)

Lindner F, Schätzel M G, Walther H, Baltuška A, Goulielmakis E, Krausz F, Milošević D B, Bauer D, Becker W and Paulus G G 2005 Attosecond double-slit experiment Phys. Rev. Lett. 95040401

Liu N, Tang M L, Hentschel M, Giessen A P and Alivisatos H 2011 Nanoantenna-enhanced gas sensing in a single tailored nanofocus Nat. Mater. 10 631-6

Locher R, Castiglioni L, Lucchini M, Greif M, Gallmann L, Osterwalder J, Hengsberger M and Keller U 2015 Energydependent photoemission delays from noble metal surfaces by attosecond interferometry Optica $\mathbf{2} 405$

Locher R et al 2014 Versatile attosecond beamline in a two-foci configuration for simultaneous time-resolved measurements Rev. Sci. Instrum. 85013113

Lorek E et al 2015 Size and shape dependent few-cycle near-field dynamics of bowtie nanoantennas Opt. Express 23 31460-71

Lotz W 1967 An empirical formula for the electron-impact ionization cross-section Z. Phys. 206 205-11

Luecking F, Assion A, Apolonski A, Krausz F and Steinmeyer G 2012 Long-term carrier-envelope-phase-stable few-cycle pulses by use of the feed-forward method Opt. Lett. 37 2076-8

Lüneburg S, Müller M, Paarmann A and Ernstorfer R 2013 Microelectrode for energy and current control of nanotip field electron emitters Appl. Phys. Lett. 103213506
Luo J, Li Y, Wang Z, He L, Zhang Q and Lu P 2013a Efficient supercontinuum generation by uv-assisted midinfrared plasmonic fields Phys. Rev. A 89023405

Luo J, Li Y, Wang Z, Zhang Q, Lan P and Lu P 2013b Wavelength dependence of high-order-harmonic yield in inhomogeneous fields J. Opt. Soc. Am. B 30 2469-75

Luo J, Li Y, Wang Z, Zhang Q and Lu P 2013c Ultra-short isolated attosecond emission in mid-infrared inhomogeneous fields without cep stabilization J. Phys. B: At. Mol. Opt. Phys. 46145602

Lupetti M, Hengster J, Uphues T and Scrinzi A 2014 Attosecond photoscopy of plasmonic excitations Phys. Rev. Lett. 113113903

Luu T T, Garg M, Kruchinin S Yu, Moulet A, Hassan M Th and Goulielmakis E 2015 Extreme ultraviolet high-harmonic spectroscopy of solids Nature $\mathbf{5 2 1} 498-502$

Maier S A 2007 Plasmonics: Fundamentals and Applications (New York: Springer)

Mairesse Y et al 2003 Attosecond synchronization of highharmonic soft X-rays Science 302 1540-3

Mairesse Y and Quéré F 2005 Frequency-resolved optical gating for complete reconstruction of attosecond bursts Phys. Rev. A 71011401

Marangos J P 2011 Introduction to the new science with x-ray free electron lasers Contemp. Phys. 52 551-69

Marangos J P 2016 Development of high harmonic generation spectroscopy of organic molecules and biomolecules $J$. Phys. B: At. Mol. Opt. Phys. 49132001

Marinica D C, Zapata M, Nordlander P, Kazansky A K, Echenique P, Aizpurua J and Borisov A G 2015 Active quantum plasmonics Sci. Adv. 1 e1501095

Martin O J F and Girard C 1997 Controlling and tuning strong optical field gradients at a local probe microscope tip apex Appl. Phys. Lett. 70 705-7

Martin Y C, Hamann H F and Wickramasinghe H K 2001 Strength of the electric field in apertureless near-field optical microscopy J. Appl. Phys. 89 5774-8

Mashiko H, Bell M J, Beck A R, Abel M J, Nagel P M, Steiner C P, Robinson J, Neumark D M and Leone S R 2010 Tunable frequency-controlled isolated attosecond pulses characterized by either $750 \mathrm{~nm}$ or $400 \mathrm{~nm}$ wavelength streak fields $O p t$. Express 18 25887-95

Mashiko H, Gilbertson S, Li C, Khan S D, Shakya M M, Moon E and Chang Z 2008 Double optical gating of high-order harmonic generation with carrier-envelope phase stabilized lasers Phys. Rev. Lett. 100103906

Mermin N D 1970 Lindhard dielectric function in the relaxationtime approximation Phys. Rev. B $12362-3$

Miaja-Avila L, Lei C, Aeschlimann M, Gland J L, Murnane M M, Kapteyn H C and Saathoff G 2006 Laser-assisted photoelectric effect from surfaces Phys. Rev. Lett. 97113604

Mikkelsen A et al 2009 Photoemission electron microscopy using extreme ultraviolet attosecond pulse trains Rev. Sci. Instrum. 80123703

Milošević D B, Paulus G G, Bauer D and Becker W 2006 Abovethreshold ionization by few-cycle pulses J. Phys. B: At. Mol. Opt. Phys. 39 R203-62

Miranda M, Fordell T, Arnold C, L'Huillier A and Crespo H 2012 Simultaneous compression and characterization of ultrashort laser pulses using chirped mirrors and glass wedges $O p t$. Express 20 688-97

Mountford L C, Smith R A and Hutchinson M H R 1998 Characterization of a sub-micron liquid spray for laser-plasma x-ray generation Rev. Sci. Instrum. 693780

Muller H G, van Linden van den Heuvell H B and van der Wiel M J 1986 Experiments on 'above-threshold ionization' of atomic hydrogen Phys. Rev. A 34236

Müller M, Kravtsov V, Paarmann A, Raschke M B and Ernstorfer R 2016 Nanofocused plasmon-driven sub-10 fs electron point source ACS Photonics 3 611-9 
Müller M, Paarmann A and Ernstorfer R 2014 Femtosecond electrons probing currents and atomic structure in nanomaterials Nat. Commun. 55292

Mustonen A, Beaud P, Kirk E, Feurer T and Tsujino S 2011 Five picocoulomb electron bunch generation by ultrafast laserinduced field emission from metallic nano-tip arrays Appl. Phys. Lett. 99103504

Mustonen A, Beaud P, Kirk E, Feurer T and Tsujino S 2012 Efficient light coupling for optically excited high-density metallic nanotip arrays Sci. Rep. 2915

Nagel P M, Robinson J S, Harteneck B D, Pfeifer T, Abel M J, Prell J S, Neumark D M, Kaindl R A and Leone S R 2013 Surface plasmon assisted electron acceleration in photoemission from gold nanopillars Chem. Phys. 414 106-11

Naik G V, Shalaev V M and Boltasseva A 2013 Alternative plasmonic materials: beyond gold and silver $A d v$. Mater. 25 3264-94

Nakamura A and Yamada S 1981 Fundamental absorption edge of evaporated amorphous WO3 films Appl. Phys. 24 55-9

Neacsu C C, Reider G A and Raschke M B 2005 Second-harmonic generation from nanoscopic metal tips: symmetry selection rules for single asymmetric nanostructures Phys. Rev. B 71201402

Nelayah J, Kociak M, Stéphan O, García de Abajo F J, Tencé M, Henrard L, Taverna D, Pastoriza-Santos I, Liz-Marzán L M and Colliex C 2007 Mapping surface plasmons on a single metallic nanoparticle Nat. Phys. 3 348-53

Neppl S, Ernstorfer R, Bothschafter E M, Cavalieri A L, Menzel D, Barth J V, Krausz F, Kienberger R and Feulner P 2012 Attosecond time-resolved photoemission from core and valence states of magnesium Phys. Rev. Lett. 109087401

Neppl S 2012 Attosecond time-resolved photoemission from surfaces and interfaces $P h D$ Thesis LMU Munich

Novotny L and Hecht B 2012 Principles of Nano-Optics (Cambridge: Cambridge University Press)

Novotny L and van Hulst N 2011 Antennas for light Nat. Photon. 5 83-90

Okell W A et al 2015 Temporal broadening of attosecond photoelectron wavepackets from solid surfaces Optica 2 383-7

Okell W A et al 2013 Carrier-envelope phase stability of hollow fibers used for high-energy few-cycle pulse generation $O p t$. Lett. 38 3918-21

Paarmann A et al 2012 Coherent femtosecond low-energy singleelectron pulses for time-resolved diffraction and imaging: a numerical study J. Appl. Phys. 112113109

Park D J, Piglosiewicz B, Schmidt S, Kollmann H, Mascheck M, Groß P and Lienau C 2013 Characterizing the optical near-field in the vicinity of a sharp metallic nanoprobe by angle-resolved electron kinetic energy spectroscopy Ann. Phys. 525 135-42

Park D J, Piglosiewicz B, Schmidt S, Kollmann H, Mascheck M and Lienau C 2012 Strong field acceleration and steering of ultrafast electron pulses from a sharp metallic nanotip Phys. Rev. Lett. 109244803

Park I-Y, Kim S, Choi J, Lee D-H, Kim Y J, Kling M F, Stockman M I and Kim S-W 2011 Plasmonic generation of ultrashort extreme-ultraviolet light pulses Nat. Photon. 5677

Paulus G G, Grasbon F, Walther H, Villoresi P, Nisoli M, Stagira S, Priori E and De Silvestri S 2001 Absolute-phase phenomena in photoionization with few-cycle laser pulses Nature 414 182-4

Paulus G G, Lindner F, Walther H, Baltuška A, Goulielmakis E, Lezius M and Krausz F 2003 Measurement of the phase of few-cycle laser pulses Phys. Rev. Lett. 91253004

Paulus G G, Lindner F, Walther H and D Milošević 2004 Phasecontrolled single-cycle strong-field photoionization Phys. Scr. $T 110120-5$

Paulus G G, Nicklich W, Huale X, Lambropoulus P and Walter H 1994 Plateau in above threshold ionization spectra Phys. Rev Lett. 722851
Pazourek R, Nagele S and Burgdörfer J 2015 Attosecond chronoscopy of photoemission Rev. Mod. Phys. 87765

Perelomov A M, Popov V S and Terentev M V 1966 Ionization of atoms in an alternating electric field Sov. Phys._JETP 23924

Pérez-Hernández J A, Ciappina M F, Lewenstein M, Roso L and Zaïr A 2013 Beyond carbon K-edge harmonic emission using a spatial and temporal synthesized laser field Phys. Rev. Lett. 110053001

Pérez-Hernández J A, Hoffmann D J, Zaïr A, Chipperfield L E, Plaja L, Ruiz C, Marangos J P and Roso L 2009 Extension of the cut-off in high-harmonic generation using two delayed pulses of the same colour J. Phys. B: At. Mol. Opt. Phys. 42134004

Pérez-Hernández J A, Roso L, Zaïr A and Plaja L 2011 Valley in the efficiency of the high-order harmonic yield at ultra-high laser intrensities Opt. Express 19 19430-9

Pfullmann N et al 2013 Bow-tie nano-antenna assisted generation of extreme ultraviolet radiation New J. Phys. 15093027

Piglosiewicz B, Schmidt S, Park D J, Vogelsang J, Gross P, Manzoni C, Farinello P, Cerullo G and Lienau C 2014 Carrierenvelope phase effects on the strong-field photoemission of electrons from metallic nanostructures Nat. Photon. 8 37-42

Prinz S et al 2015 Cep-stable, sub-6 fs, $300 \mathrm{kHz}$ opcpa system with more than $15 \mathrm{~W}$ of average power Opt. Express 23 1388-94

Pullen M G et al 2015 Imaging an aligned polyatomic molecule with laser-induced electron diffraction Nat. Commun. 67262

Qin D, Xia Y and Whitesides G M 2010 Soft lithography for microand nanoscale patterning Nat. Protocols 5 491-502

Quinonez E, Handali J and Barwick B 2013 Femtosecond photoelectron point projection microscope Rev. Sci. Instr. 84103710

Rathje T, Johnson N G, Müller M, Süßmann F, Adolph D, Kübel M, Kienberger R, Kling M F, Paulus G G and Sayler A M 2012 Review of attosecond resolved measurement and control via carrier-envelope phase tagging with above-threshold ionization J. Phys. B: At. Mol. Opt. Phys. 45074003

Reiss H R 1980 Effect of an intense electromagnetic field on a weakly bound system Phys. Rev. A 221786

Rewitz C, Keitzl T, Tuchscherer P, Huang J-S, Geisler P, Razinskas G, Hecht B and Brixner T 2012 Ultrafast plasmon propagation in nanowires characterized by far-field spectral interferometry Nano Lett. 12 45-9

Robinson J S, Haworth C A, Teng H, Smith R A, Marangos J P and Tisch J W G 2006 The generation of intense, transformlimited laser pulses with tunable duration from 6 to $30 \mathrm{fs}$ in a differentially pumped hollow fibre Appl. Phys. B 85 525-9

Ropers C, Elsässer T, Cerullo G, Zavelani-Rossi M and Lienau C 2007a Ultrafast optical excitations of metallic nanostructures: from light confinement to a novel electron source New J. Phys. 9397

Ropers C, Solli D R, Schulz C-P, Lienau C and Elsässer T 2007b Localized multiphoton emission of femtosecond electron pulses from metal nanotips Phys. Rev. Lett. 98043907

Salières P et al 2001 Feynman's path-integral approach for intenselaser-atom interactions Science 292 902-5

Salières P, L'Huillier A, Antoine P and Lewenstein M 1999 Study of the spatial and temporal coherence of high-order harmonics Advances in Atomic, Molecular and Optical Physics vol 41, ed B Bederson and H Walter (San Diego, CA: Academic) pp $83-142$

Salvat F, Jablonski A and Powell C J 2005 Elsepa-dirac partialwave calculation of elastic scattering of electrons and positrons by atoms, positive ions and molecules Comput. Phys. Commun. 165 157-90

Sansone $\mathrm{G}$ et al 2006 Isolated single-cycle attosecond pulses Science 314 443-6

Santra R and Gordon A 2006 Three-step model for high-harmonic generation in many-electron systems Phys. Rev. Lett. 96073906 
Sarid D and Challener W 2010 Modern Introduction to Surface Plasmons (Cambridge: Cambridge University Press)

Sau T K and Murphy C J 2004 Room temperature, high-yield synthesis of multiple shapes of gold nanoparticles in aqueous solution J. Am. Chem. Soc. 126 8648-9

Savage K J, Hawkeye M M, Esteban R, Borisov A G, Aizpurua J and Baumberg J J 2012 Revealing the quantum regime in tunnelling plasmonics Nature 491 574-7

Sayler A M, Rathje T, Müller W, Rühle K, Kienberger R and Paulus G G 2011 Precise, real-time, every-single-shot, carrierenvelope phase measurement of ultrashort laser pulses Opt. Lett 36 1-3

Schafer K J 1991 The energy analysis of time-dependent numerical wave functions Comput. Phys. Commun. 63 427-34

Schafer K J 2009 Numerical methods in strong field physics Strong Field Laser Physics ed T Brabec (New York: Springer) pp 111-45

Schafer K J, Yang B, DiMauro L F and Kulander K C 1993 Above threshold ionization beyond the high harmonic cutoff Phys. Rev. Lett. 701599

Schafer K J and Kulander K C 1990 Energy analysis of timedependent wave functions: application to above-threshold ionization Phys. Rev. A 425794

Schenk M, Krüger M and Hommelhoff P 2010 Strong-field abovethreshold photoemission from sharp metal tips Phys. Rev. Lett. 105257601

Schenk M, Krüger M and Hommelhoff P 2011 Carrier-envelope phase dependent photoemission from a nanometric metal tip Joint Conf. of the IEEE Int. Frequency Control and the European Frequency and Time Forum (IEEE) pp 404-7

Schertz F, Schmelzeisen M, Kreiter M, Elmers H-J and Schönhense G 2012 Field emission of electrons generated by the near field of strongly coupled plasmons Phys. Rev. Lett 108237602

Schiffrin A et al 2013 Optical-field-induced current in dielectrics Nature 493 70-4

Schmidt B E, Shiner A D, Lassonde P, Kieffer J-C, Corkum P B, Villeneuve D M and Légaré F 2011 Cep stable 1.6 cycle laser pulses at $1.8 \mu \mathrm{m}$ Opt. Express $196858-64$

Scholl J A, García-Etxarri A, Koh A L and Dionne J A 2013 Observation of quantum tunneling between two plasmonic nanoparticles Nano Lett. 13 564-9

Scholz F, Himmel D, Heinemann F W, Schleyer P v R, Meyer K and Krossing I 2013 Crystal structure determination of the nonclassical 2-norbornyl cation Science 341 62-4

Schröder B, Sivis M, Bormann R, Schäfer S and Ropers C 2015a An ultrafast nanotip electron gun triggered by grating-coupled surface plasmons Appl. Phys. Lett. 107231105

Schröder B et al 2015b Real-space imaging of nanotip plasmons using electron energy loss spectroscopy Phys. Rev. B 92085411

Schubert O et al 2014 Sub-cycle control of terahertz high-harmonic generation by dynamical bloch oscillations Nat. Photon. 8 119-23

Schultze M et al 2013 Controlling dielectrics with the electric field of light Nature 493 75-8

Schultze M et al 2010 Delay in photoemission Science 328 1658-62

Schweinberger W, Sommer A, Bothschafter E, Li J, Krausz F, Kienberger R and Schultze M 2012 Waveform-controlled near-single-cycle milli-joule laser pulses generate sub- $10 \mathrm{~nm}$ extreme ultraviolet continua Opt. Lett. 37 3573-5

Scrinzi A, Ivanov M Y, Kienberger R and Villeneuve D M 2006 Attosecond physics J. Phys. B: At. Mol. Opt. Phys. 39 R1-37

Seiffert L, Süßmann F, Zherebtsov S, Rupp P, Peltz C, Rühl E, Kling M F and Fennel T 2016 Competition of single and double rescattering in the strong-field photoemission from dielectric nanospheres Appl. Phys. B 122101

Shaaran T, Ciappina M F, Guichard R, Pérez-Hernández J A, Roso L, Arnold M, Siegel T, Zaïr A and Lewenstein M 2013a
High-order-harmonic generation by enhanced plasmonic nearfields in metal nanoparticles Phys. Rev. A 87041402

Shaaran T, Ciappina M F and Lewenstein M 2012a Estimating the plasmonic field enhancement using high-order harmonic generation: the role of the field inhomogeneity $J$. Mod. Opt. 59 1634-9

Shaaran T, Ciappina M F and Lewenstein M 2012b Quantumorbit analysis of high-order-harmonic generation by resonant plasmon field enhancement Phys. Rev. A 86023408

Shaaran T, Ciappina M F and Lewenstein M 2013b Quantum-orbit analysis of above-threshold ionization driven by an intense spatially inhomogeneous field Phys. Rev. A 87053415

Shao Y L, Ditmire T, Tisch J W G, Springate E, Marangos J P and Hutchinson M H R 1996 Multi-kev electron generation in the interaction of intense laser pulses with xe clusters Phys. Rev. Lett. 773343

Shi-Lin H and Ting-Yun S 2013 Effect of electron correlation on high-order harmonic generation in helium model atom Chin. Phys. B 22013101

Shiner A D, Schmidt B E, Trallero-Herrero C, Wörner H J, Patchkovskii S, Corkum P B, Kieffer J-C, Légaré F and Villeneuve D M 2011 Probing collective multi-electron dynamics in xenon with high-harmonic spectroscopy Nat. Phys. 7 464-7

Sivis M, Duwe M, Abel B and Ropers C 2012 Nanostructureenhanced atomic line emission Nature 485 E1-3

Sivis M, Duwe M, Abel B and Ropers C 2013 Extreme-ultraviolet light generation in plasmonic nanostructures Nat. Phys. 9 304-9

Sivis M and Ropers C 2013 Generation and bistability of a waveguide nanoplasma observed by enhanced extremeultraviolet fluorescence Phys. Rev. Lett. 111085001

Skopalová E, El-Taha Y C, Zaïr A, Hohenberger M, Springate E, Tisch J W G, Smith R A and Marangos J P 2010 Pulse-length dependence of the anisotropy of laser-driven cluster explosions: transition to the impulsive regime for pulses approaching the few-cycle limit Phys. Rev. Lett. 104203401

Skopalová E, Lei D Y, Witting T, Arrell C, Frank F, Sonnefraud Y, Maier S A, Tisch J W G and Marangos J P 2011 Numerical simulation of attosecond nanoplasmonic streaking New J. Phys. 13083003

Smirnova O, Mairesse Y, Patchkovskii S, Dudovich N, Villeneuve D, Corkum P B and Ivanov M Yu 2009 High harmonic interferometry of multi-electron dynamics in molecules Nature $460972-7$

Smith R A, Ditmire T and Tisch J W G 1998 Characterization of a cryogenically cooled high-pressure gas jet for laser/cluster interaction experiments Rev. Sci. Instrum. 693798

Sola I J et al 2006 Controlling attosecond electron dynamics by phase-stabilized polarization gating Nat. Phys. $2319-22$

Solleder B, Lemell C, Tőkési K, Hatcher N and Burgdörfer J 2007 Spin-dependent low-energy electron transport in metals Phys. Rev. B 76075115

Sonnefraud Y, Leen Koh A, McComb D W and Maier S A 2012 Nanoplasmonics: engineering and observation of localized plasmon modes Laser Photonics Rev. 6 277-95

Sönnichsen C, Franzl T, Wilk T, von Plessen G, Feldmann J, Wilson O and Mulvaney P 2002 Drastic reduction of plasmon damping in gold nanorods Phys. Rev. Lett. 88077402

Stebbings S L, Süßmann F, Yang Y-Y, Scrinzi A, Durach M, Rusina A, Stockman M I and Kling M F 2011 Generation of isolated attosecond extreme ultraviolet pulses employing nanoplasmonic field enhancement: optimization of coupled ellipsoids New J. Phys. 13073010

Stöber W, Fink A and Bohn E 1968 Controlled growth of monodisperse silica spheres in the micron size range $J$. Coll. Int. Sci. 26 62-9

Stöckle Raoul M, Doug S Y, Volker D and Renato Z 2000 Nanoscale chemical analysis by tip-enhanced Raman spectroscopy Chem. Phys. Lett. 318 131-6 
Stockman M I 2011 Nanoplasmonics: past, present, and glimpse into future Opt. Express 19 22029-106

Stockman M I, Kling M F, Kleineberg U and Krausz F 2007 Attosecond nanoplasmonic-field microscope Nat. Photon. $1539-44$

Sumeruk H A, Kneip S, Symes D R, Churina I V, Belolipetski A V, Donnelly T D and Ditmire T 2007a Control of strong-laserfield coupling to electrons in solid targets with wavelengthscale spheres Phys. Rev. Lett. 98045001

Sumeruk H A et al 2007b Hot electron and x-ray production from intense laser irradiation of wavelength-scale polystyrene spheres Phys. Plasmas 14062704

Süßmann F 2013 Attosecond dynamics of nano-localized fields probed by photoelectron spectroscopy PhD Thesis LMU Munich

Süßmann F and Kling M F 2011a Attosecond measurement of petahertz plasmonic near-fields Proc. SPIE $809680961 \mathrm{C}$

Süßmann F and Kling M F 2011b Attosecond nanoplasmonic streaking of localized fields near metal nanospheres Phys. Rev. B 84121406

Süßmann F et al 2015 Field propagation-induced directionality of carrier-envelope phase-controlled photoemission from nanospheres Nat. Commun. 67944

Süßmann F, Stebbings S L, Zherebtsov S, Chew S H, Stockman M I, Rühl E, Kleineberg U, Fennel T and Kling M F 2014 Attosecond nanophysics Attosecond and XUV Physics (New York: Wiley) pp 421-62

Süßmann F et al 2011 Single-shot velocity-map imaging of attosecond light-field control at kilohertz rate Rev. Sci. Instrum. 82093109

Swanwick M E, Keathley P D, Fallahi A, Krogen P R, Laurent G, Moses J, Kärtner F X and Velásquez-García L F 2014 Nanostructured ultrafast silicon-tip optical field-emitter arrays Nano Lett. 14 5035-43

Symes D R, Comley A J and Smith R A 2004 Fast-ion production from short-pulse irradiation of ethanol microdroplets Phys. Rev. Lett. 93145004

Taflove A and Hagness S C 2005 Computational Electrodynamics: the Finite-Difference Time-Domain Method (Boston: Artech House)

Tanuma S, Powell C J and Penn D R 2011 Calculations of electron inelastic mean free paths. IX. Data for 41 elemental solids over the $50 \mathrm{eV}$ to $30 \mathrm{keV}$ range Surf. Interface Anal. 43 689-713

Tanuma S, Shiratori T, Kimura T, Goto K, Ichimura S and Powell C J 2005 Experimental determination of electron inelastic mean free paths in 13 elemental solids in the $50-5000 \mathrm{eV}$ energy range by elastic-peak electron spectroscopy Surf. Interface Anal. 37 833-45

Teichmann S M, Rácz P, Ciappina M F, Pérez-Hernández J A, Thai A, Fekete J, Elezzabi A Y, Veisz L, Biegert J and Dombi P 2015 Strong-field plasmonic photoemission in the mid-IR at $<1 \mathrm{GW} \mathrm{cm}^{-2}$ intensity Sci. Rep. 57584

Tőkési K, Wirtz L, Lemell C and Burgdörfer J 2001 Hollow-ion formation in microcapillaries Phys. Rev. A 64042902

Thomas S, Krüger M, Förster M, Schenk M and Hommelhoff P 2013 Probing of optical near-fields by electron rescattering on the $1 \mathrm{~nm}$ scale Nano Lett. 13 4790-4

Thomas S, Wachter G, Lemell C, Burgdörfer J and Hommelhoff P 2015 Large optical field enhancement for nanotips with large opening angles New. J. Phys. 17063010

Tikman Y, Yavuz I, Ciappina M F, Chacón A, Altun Z and Lewenstein M 2016 High-order-harmonic generation from rydberg atoms driven by plasmon-enhanced laser fields Phys. Rev. A 93023410

Tisch J W G, Ditmire T, Fraser D J, Hay N, Mason M B, Springate E, Marangos J P and Hutchinson M H R 1997 Investigation of high-harmonic generation from xenon atom clusters J. Phys. B: At. Mol. Opt. Phys. 30 L709

Tóth C, Farkas G and Vodopyanov K L 1991 Laser-induced electron emission from an au surface irradiated bey single picosecond pulses at $\lambda=2.94 \mu \mathrm{m}$. the intermediate region between multiphoton and tunneling effect Appl. Phys. B 53 221-5

Trebino R, DeLong K W, Fittinghoff D N, Sweetser J N, Krumbügel M A, Richman B A and Kane D J 1997 Measuring ultrashort laser pulses in the time-frequency domain using frequency-resolved optical gating Rev. Sci. Instrum. 68 3277-95

Tsujino S, Beaud P, Kirk E, Vogel T, Sehr H, Gobrecht J and Wrulich A 2008 Ultrafast electron emission from metallic nanotip arrays induced by near infrared femtosecond laser pulses Appl. Phys. Lett. 92193501

Tsujino S, le Pimpec F, Raabe J, Buess M, Dehler M, Kirk E, Gobrecht J and Wrulich A 2009 Static and optical field enhancement in metallic nanotips studied by two-photon photoemission microscopy and spectroscopy excited by picosecond laser pulses Appl. Phys. Lett. 94093508

Uiberacker M et al 2007 Attosecond real-time observation of electron tunnelling in atoms Nature 446 627-32

Uphues Th, Schultze M, Kling M F, Uiberacker M, Hendel S, Heinzmann U, Kabachnik N M and Drescher M 2008 Ioncharge-state chronoscopy of cascaded atomic auger decay $\mathrm{New}$ J. Phys. 10025009

Vampa G, Hammond T J, Thiré N, Schmidt B E, Légaré F, McDonald C R, Brabec T and Corkum P B 2015 Linking high harmonics from gases and solids Nature $\mathbf{5 2 2} 462-4$

Veronis G and Fan S 2007 Overview of simulation techniques for plasmonic devices Surface Plasmon Nanophotonics (Springer Series in Optical Sciences vol 131) ed M L Brongersma and P G Kik (Netherlands: Springer) pp 169-82

Vesseur E J R, de Waele R, Kuttge M and Polman A 2007 Direct observation of plasmonic modes in au nanowires using highresolution cathodoluminescence spectroscopy Nano Lett. $72843-6$

Vogelsang J, Robin J, Nagy B J, Dombi P, Rosenkranz D, Schiek M, Groß P and Lienau C 2015 Ultrafast electron emission from a sharp metal nanotaper driven by adiabatic nanofocusing of surface plasmons Nano Lett. 15 4685-91

Wachter G, Lemell C, Burgdörfer J, Schenk M, Krüger M and Hommelhoff P 2012 Electron rescattering at metal nanotips induced by ultrashort laser pulses Phys. Rev. B 86035402

Walker B, Sheehy B, DiMauro L F, Agostini P, Schafer K J and Kulander K C 1994 Precision measurement of strong field double ionization of helium Phys. Rev. Lett. 731227

Walmsley I A and Dorrer C 2009 Characterization of ultrashort electromagnetic pulses Adv. Opt. Photonics 1 308-437

Wang Z, He L, Luo J, Lan P and Lu P 2014 High-order harmonic generation from rydberg atoms in inhomogeneous fields Opt. Express 22 25909-22

Wang Z, Lan P, Luo J, He L, Zhang Q and Lu P 2013 Control of electron dynamics with a multicycle two-color spatially inhomogeneous field for efficient single-attosecond-pulse generation Phys. Rev. A $\mathbf{8 8} 063838$

Wanzenboeck H D and Waid S 2011 Focused ion beam lithography Recent Advances in Nanofabrication Techniques and Applications ed B Cui (Rijeka: InTech) pp 27-50

Weber S J et al 2015 Flexible attosecond beamline for high harmonic spectroscopy and xuv/near-ir pump probe experiments requiring long acquisition times Rev. Sci. Instrum. 86033108

Wessel J 1985 Surface-enhanced optical microscopy J. Opt. Soc. Am. B 2 1538-41

Wimmer L, Herink G, Solli D R, Yalunin S V, Echternkamp K E and Ropers C 2014 Terahertz control of nanotip photoemission Nat. Phys. 10432

Winkel M, Speck R, Hübner H, Arnold L, Krause R and Gibbon P 2012 A massively parallel, multi-disciplinary barnes-hut tree code for extreme-scale n-body simulations Comput. Phys. Commun. 183 880-9

Wirth A et al 2011 Synthesized light transients Science 334 195-200 
Witting T, Frank F, Arrell C A, Okell W A, Marangos J P and Tisch J W G 2011 Characterization of high-intensity sub-4 fs laser pulses using spatially encoded spectral shearing interferometry Opt. Lett. 36 1680-2

Witting T, Frank F, Okell W A, Arrell C A, Marangos J P and Tisch J W G 2012 Sub-4 fs laser pulse characterization by spatially resolved spectral shearing interferometry and attosecond streaking J. Phys. B: At. Mol. Opt. Phys. 45074014

Wittmann T, Horvath B, Helml W, Schätzel M G, Gu X, Cavalieri A L, Paulus G G and Kienberger R R 2009 Singleshot carrier-envelope phase measurement of few-cycle laser pulses Nat. Phys. 5 357-62

Wolf D, Schumacher T and Lippitz M 2016 Shaping the nonlinear near field Nat. Commun. 710361

Wyatt A S, Witting T, Schiavi A, Fabris D, Matia-Hernando P, Walmsley I A, Marangos J P and Tisch J W G 2016 Attosecond sampling of arbitrary optical waveforms Optica 3 303-10

Xu J, Blaga C I, Zhang K, Lai Y H, Lin C D, Miller T A, Agostini P and DiMauro L F 2014 Diffraction using laser-driven broadband electron wave packets Nat. Commun. 54635

Xu L, Spielmann Ch, Poppe A, Brabec T, Krausz F and Hänsch T W 1996 Route to phase control of ultrashort light pulses Opt. Lett. 21 2008-10

Yakovlev V, Stockman M I, Krausz F and Baum P 2015 Atomicscale diffractive imaging of sub-cycle electron dynamics in condensed matter Sci. Rep. 514581

Yalunin S V, Gulde M and Ropers C 2011 Strong-field photoemission from surfaces: Theoretical approaches Phys. Rev. B 84195426

Yalunin S V, Herink G, Solli D R, Krüger M, Hommelhoff P, Diehn M, Munk A and Ropers C 2013 Field localization and rescattering in tip-based photoemission Ann. Phys. 525 L12-8

Yanagisawa H, Hafner C, Doná P, Klöckner M, Leuenberger D, Greber T, Hengsberger M and Osterwalder J 2009 Optical control of field-emission sites by femtosecond laser pulses Phys. Rev. Lett. 103257603

Yanagisawa H, Hafner C, Doná P, Klöckner M, Leuenberger D, Greber T, Osterwalder J and Hengsberger M 2010 Laserinduced field emission from a tungsten tip: optical control of emission sites and the emission process Phys. Rev. B 81115429

Yanagisawa H, Hengsberger M, Leuenberger D, Klöckner M, Hafner C, Greber T and Osterwalder J 2011 Energy distribution curves of ultrafast laser-induced field emission and their implications for electron dynamics Phys. Rev. Lett. 107087601

Yanagisawa H, Schnepp S, Hafner C, Hengsberger M, Kim D E, Kling M F, Landsman A S, Gallmann L and Osterwalder J 2016 Delayed electron emission in strong-field driven tunnelling from a metallic nanotip in the multi-electron regime Sci. Rep. 635877
Yavuz I 2013 Gas population effects in harmonic emission by plasmonic fields Phys. Rev. A 87053815

Yavuz I, Bleda E A, Altun Z and Topcu T 2012 Generation of a broadband XUV continuum in high-order-harmonic generation by spatially inhomogeneous fields Phys. Rev. A 85013416

Yavuz I, Ciappina M F, Chacón A, Altun Z, Kling M F and Lewenstein M 2016 Controlling electron localization in $\mathrm{H}_{2}^{+}$by intense plasmon-enhanced laser fields Phys. Rev. A 93033404

Yavuz I, Tikman Y and Altun Z 2015 High-order-harmonic generation from $\mathrm{H}_{2}^{+}$molecular ions near plasmon-enhanced laser fields Phys. Rev. A 92023413

Yu C, Wang Y, Cao X, Jiang S and Lu R 2015 Isolated fewattosecond emission in a multi-cycle asymmetrically nonhomogeneous two-color laser field J. Phys. B: At. Mol. Opt. Phys. 47225602

Yudin G L and Ivanov M Yu 2001 Nonadiabatic tunnel ionization: looking inside a laser cycle Phys. Rev. A 64013409

Zagoya C, Bonner M, Chomet H, Slade E and Figueira de Morisson Faria C 2016 Different time scales in plasmonically enhanced high-order-harmonic generation Phys. Rev. A 93053419

Zang C, Lui C and Xu Z 2013 Control of higher spectral components by spatially inhomogeneous fields in quantum wells Phys. Rev. A $\mathbf{8 8} 035805$

Zhang C-H and Thumm U 2009 Attosecond photoelectron spectroscopy of metal surfaces Phys. Rev. Lett. 102123601

Zhang C-H and Thumm U 2011a Effect of wave-function localization on the time delay in photoemission from surfaces Phys. Rev. A 84065403

Zhang C-H and Thumm U 2011b Streaking and Wigner time delays in photoemission from atoms and surfaces Phys. Rev. A 84033401

Zhang G-Y, Chen Y, Qi W-K and Qing S-M 2009 Four-state rockpaper-scissors games in constrained newman-watts networks Phys. Rev. E 79062901

Zhao K, Zhang Q, Chini M, Wu Y, Wang X and Chang Z 2012 Tailoring a 67 attosecond pulse through advantageous phasemismatch Opt. Lett. 37 3891-3

Zherebtsov S et al 2011 Controlled near-field enhanced electron acceleration from dielectric nanospheres with intense few-cycle laser fields Nat. Phys. 7 656-62

Zherebtsov S et al 2012 Carrier-envelope phase-tagged imaging of the controlled electron acceleration from $\mathrm{SiO}_{2}$ nanospheres in intense few-cycle laser fields New J. Phys. 14075010

Zuloaga J, Prodan E and Nordlander P 2009 Quantum description of the plasmon resonances of a nanoparticle dimer Nano Lett. 9 887-91

Zuloaga J, Prodan E and Nordlander P 2010 Quantum plasmonics: optical properties and tunability of metallic nanorods ACS Nano 4 5269-76 